INTERNATIONAL MONETARY FUND

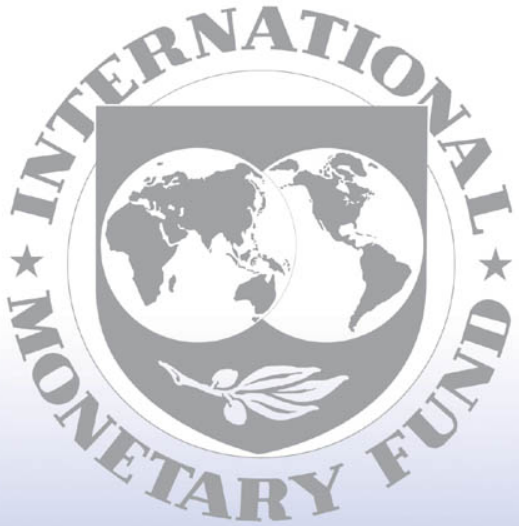

Staff

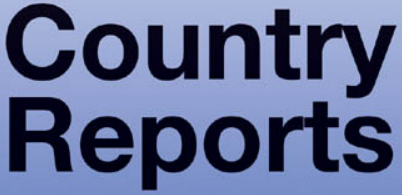




\section{Former Yugoslav Republic of Macedonia: Request for Stand-By Arrangement and Extension of Repurchase Expectations-Staff Report; Staff Statement; Press Release on the Executive Board Discussion; and Statement by the Executive Director for the former Yugoslav Republic of Macedonia}

In the context of the request for Stand-By Arrangement and extension of repurchase expectations with the former Yugoslav Republic of Macedonia, the following documents have been released and are included in this package:

- $\quad$ the staff report for the Request for Stand-By Arrangement and Extension of Repurchase Expectations, prepared by a staff team of the IMF, following discussions that ended on May 24, 2005, with the officials of the former Yugoslav Republic of Macedonia on economic developments and policies. Based on information available at the time of these discussions, the staff report was completed on August 17, 2005. The views expressed in the staff report are those of the staff team and do not necessarily reflect the views of the Executive Board of the IMF.

- a staff statement of August 31, 2005 updating information on recent economic developments.

- $\quad$ a Press Release summarizing the views of the Executive Board as expressed during its August 31, 2005 discussion of the staff report.

- $\quad$ a statement by the Executive Director for the former Yugoslav Republic of Macedonia.

The documents listed below have been or will be separately released.

Letter of Intent sent to the IMF by the authorities of the former Yugoslav Republic of Macedonia* Memorandum of Economic and Financial Policies by the authorities by the former Yugoslav Republic of Macedonia*

Technical Memorandum of Understanding*

* May also be included in the staff report

The policy of publication of staff reports and other documents allows for the deletion of market-sensitive information.

To assist the IMF in evaluating the publication policy, reader comments are invited and may be sent by e-mail to publicationpolicy@imf.org.

Copies of this report are available to the public from

International Monetary Fund $\bullet$ Publication Services

$70019^{\text {th }}$ Street, N.W. • Washington, D.C. 20431

Telephone: (202) 623-7430 • Telefax: (202) 623-7201

E-mail: publications@imf.org Internet: http://www.imf.org

Price: $\$ 15.00$ a copy

\section{International Monetary Fund}

Washington, D.C.

(C) International Monetary Fund. Not for Redistribution 
INTERNATIONAL MONETARY FUND

FORMER YUGOSLAV REPUBLIC OF MACEDONIA

\section{Request for Stand-By Arrangement and Extension of Repurchase Expectations}

Prepared by the European Department

Approved by Susan Schadler and Donal Donovan

August 17, 2005

Stand-By Arrangement. To support their three year economic program, the FYR Macedonian authorities are requesting a three-year SDR 51.7 million Stand-By Arrangement (75 percent of quota). An initial purchase of SDR 10.5 million becomes available upon approval of this request. The authorities do not plan to make subsequent purchases under the arrangement. Total access under the previous SBA was SDR 20 million, all of which was purchased.

Extension of Repurchase Expectations: To smooth the debt service profile, the authorities are requesting that the repurchase expectations for SDR 5.4 million falling due before end-September 2006 be extended to the obligations basis.

Discussions were held in Skopje during October 12-26, 2004, February 9-24, 2005, and May 10-24, 2005. The staff team held discussions with President Crvenkovski, Prime Minister Bučkovski, Finance Minister Popovski, Ministers of Labor Manasievski and Jakimovski; Minister of Economy Besimi, Minister of Justice Mladenovska, Minister of Health Panovski, National Bank of the Republic of Macedonia Governor Gošev.

Staff. The team comprised Franek Rozwadowski (head), Christine Dieterich, Heikki Hatanpaa, Elina Ribakova and Anita Tuladhar (all EUR), Karl Driessen (MFD), Boileau Loko and Eva Gutierrez (both PDR), Nadia Rendak (LEG), John Brondolo (FAD) and Kevin Ross (Resident Representative). 
Executive Summary

I. Introduction

II. The Political and Economic Background …………...................................................

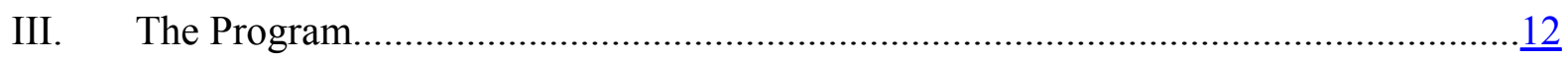

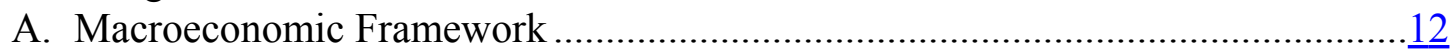

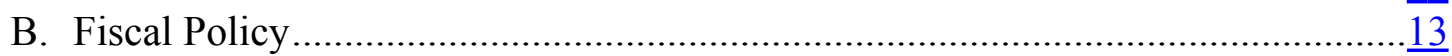

C. Monetary and Exchange Rate Policies ............................................................

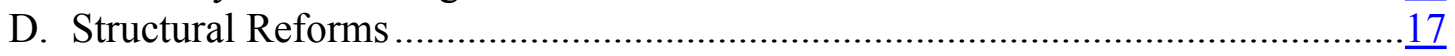

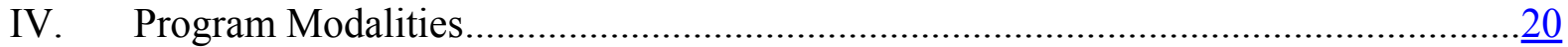

A. Type of Program and Proposed Access ……………..........................................20

B. Program Conditionality and Monitoring ...........................................................

C. Capacity to Repay the Fund and Program Risks ……………………………........

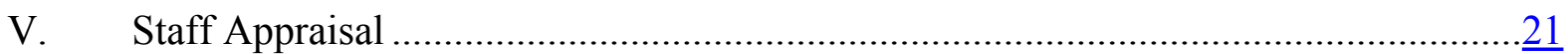

Text Tables

1. Summary of Economic Indicators, 2000-05 ............................................................

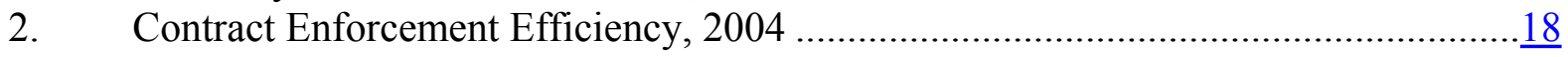

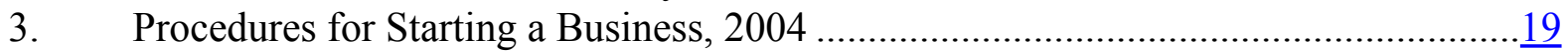

\section{Text Charts}

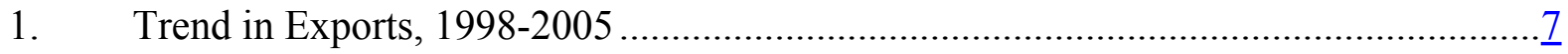

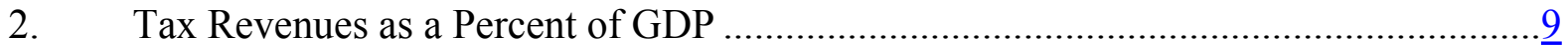

3. Amortization, Budget Support and Net Issuance of T-Bills .........................................

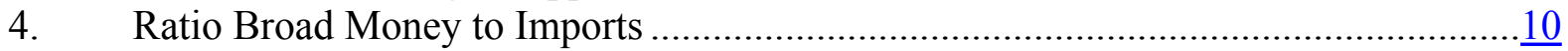

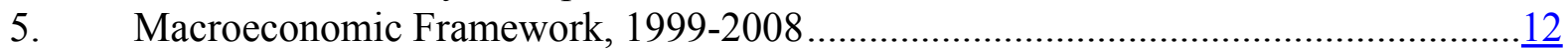

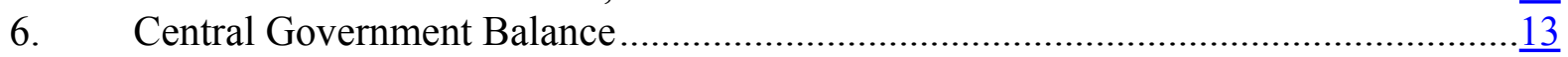

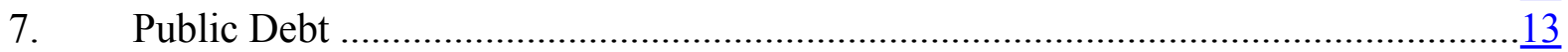

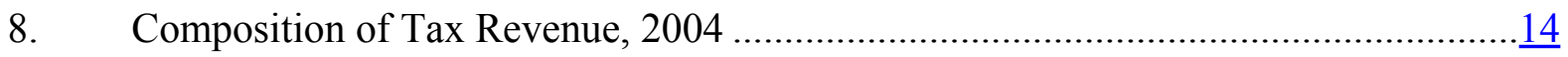

Text Boxes

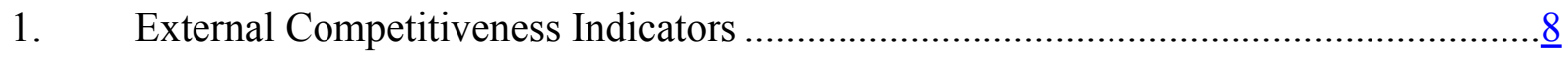

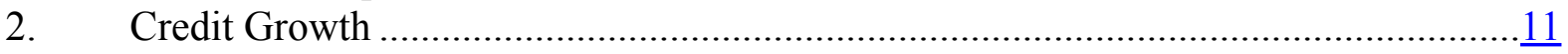

Figures

1. Cross-Country Comparison of Selected Economic Indicators ……….......................24

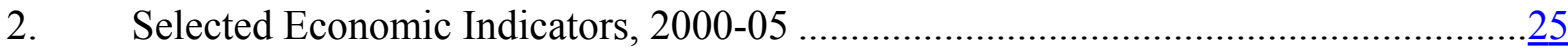


3. Monetary and Financial Indicators, 2000-05 ..................................................26

4. External Sector Development and Competitiveness, 2000-05 ..............................27

5. Recent Developments and Medium-Term Projections, 2000-09........................... 28

Tables

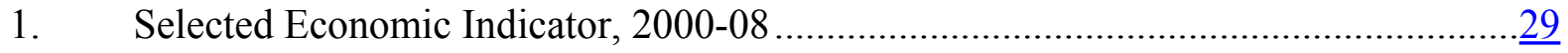

2. Central Government Operations, 2000-08 ....................................................... $\frac{30}{32}$

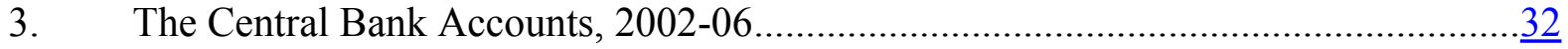

4. Monetary Survey, 2002-06 ..........................................................................

5. Medium-Term Balance of Payments, 2000-09 .................................................34

6. Macroeconomic Framework, 1999-2008 …......................................................35

7. Schedule of Purchases Under the Stand-By Arrangement ......................................

8. External Financing Requirements and Sources, 2003-08 .....................................

9. Indicators of Capacity to Repay the Fund, 2003-10 ........................................

10. Projected Payments to the Fund as of April 30, 2005 Under Obligated

Repurchase Schedule .......................................................................................

11. Indicators of Financial and External Vulnerability, 2000-05 ................................

Appendices

I. Letter of Intent ...................................................................................

Attachment 1. Memorandum on Economic and Financial Policies............................ 42

Table 1. Quantitative Performance Criteria and Indicative Targets

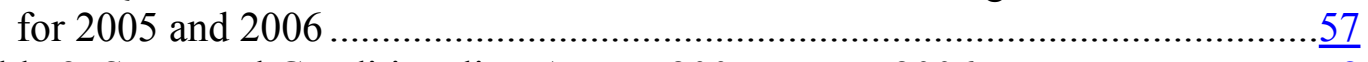

Table 2. Structural Conditionality, August 2005 to June 2006 .............................58

Table 3. Summary and Timetable for Potential Structural Measures ......................59

Attachment 2. Technical Memorandum of Understanding.....................................63

Table 1. Net International Reserves of the NBRM...........................................

Table 2. Balance of Payments Finance Assumptions, from end-December 2004

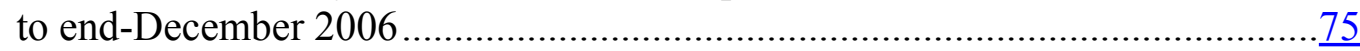

II. Debt Sustainability Analysis.....................................................................76

Table 1. Fiscal Debt Sustainability Framework, 2000-10 .................................. $\frac{80}{81}$

Table 2. External Debt Sustainability Framework, 2000-10 .............................. $\frac{81}{82}$

III. Financial Position in the Fund ..................................................................

IV. Statistical Issues .....................................................................................

Table of Common Indicators Required for Surveillance...................................... $\underline{88}$

V. IMF-World Bank Relations ................................................................... $\frac{89}{95}$

VI. Selected Social and Demographic Indicators ................................................ $\frac{95}{96}$

VII. Millennium Development Goals................................................................ $\underline{96}$ 


\section{ABBREVIATIONS AND ACRONYMS}

DSA Debt Sustainability Analysis

EAR

European Agency for Reconstruction

EPA

Ex Post Assessment

EBRD European Bank for Reconstruction and Development

EU

FA

European Union

FAD

Framework Agreement

FDI

Fiscal Affairs Department

FSAP

Foreign Direct Investment

GDP

Financial Sector Assessment Program

HIF

Gross Domestic Product

LOLR

Health Insurance Fund

MEFP

Law on Labor Relations

MFD

Memorandum on Economic and Financial Policies

MOF

Monetary and Financial Department

$\mathrm{MOH}$

Ministry of Finance

NATO

Ministry of Health

NBRM

North Atlantic Treaty Organization

PDF

PRO

National Bank of the Republic of Macedonia

Pension and Disability Fund

PHI

Public Revenue Office

SBA

Public Health Institution

USAID U.S. Agency for International Development

WTO

World Trade Organization 


\section{EXECUTIVE SUMMARY}

FYR Macedonia's impressive record of macroeconomic stabilization has been marred by a lack of comparable progress in structural reforms. Fiscal discipline has kept the debt ratio low while the de facto exchange rate peg has brought inflation close to zero. However, poorly functioning institutions - restrictive labor market regulations, inefficient state institutions and dysfunctional courts - have undermined business activity directly and kept per capita FDI low, even by regional standards. The resulting weak competitiveness has led to disappointing growth, high unemployment, and a persistent current account deficit. The restrictive business climate has also created a large informal sector. A currency devaluation in 1997 did little to improve performance, underlining that the problem was incomplete structural reforms.

\section{Against this background the authorities have asked the Fund to support an ambitious program which includes structural reforms that address the root causes of FYR Macedonia's weak performance. The main structural reforms affecting the business climate are a new, more flexible Law on Labor Relations and an overhaul of the judicial system. In addition, banks will be made more resilient by strengthening the fit and proper rules and improving the supervision of foreign exchange exposures.}

On the macroeconomic front, the near-term aims of the program are to strengthen the exchange rate peg by increasing foreign exchange reserves and to manage the transition from official budget financing (which is drying up) to market-based financing (which is still nascent). The program relies on proceeds from two large privatizations to build up reserve cover in the first two years and on sound macroeconomic policies to maintain reserve cover thereafter. The government's capacity to raise budget finance on the market will be increased by making the government debt market deeper and more liquid, keeping debt under a tight rein in order to sustain market confidence, and making an initial, cautious, eurobond issue.

The program also aims to secure medium term fiscal sustainability in the face of pressures on both expenditure and revenue. A reform of the Health Insurance Fund will address one source of risk by improving governance in a sector with large outlays; tax administration reform will broaden the base for payroll taxes; and expenditure control will be improved. A ceiling on wages will help ensure that the costs of key reforms (including Framework Agreement-related reforms) are kept within affordable limits. If successful, the fiscal reforms will create room, later in the program, to cut payroll taxes, further improving conditions for investment and employment.

The program, which will become precautionary after the first purchase, is expected to create the basis for an orderly exit from IMF support. 


\section{INTRODUCTION}

\section{In the $\mathbf{1 4}$ years since independence FYR Macedonia achieved a sustained} stabilization of demand but did not make comparable progress on structural reforms.

On the demand side, fiscal discipline and a peg to the euro brought inflation close to zero and set the government debt ratio on a declining path. But, on the supply side, early progress in dismantling centralized planning institutions was followed by delays and missteps in

\begin{tabular}{|c|c|c|c|c|c|c|}
\hline \multicolumn{7}{|c|}{ Text Table 1: Summary of Economic Indicators, 2000-2005 } \\
\hline & 2000 & 2001 & 2002 & 2003 & 2004 & 2005 \\
\hline & \multicolumn{6}{|c|}{ (In percent) } \\
\hline Real GDP & 4.5 & -4.5 & 0.9 & 2.8 & 2.9 & 3.7 \\
\hline \multirow[t]{2}{*}{ Inflation } & 5.8 & 5.3 & 2.4 & 1.2 & -0.3 & 1.2 \\
\hline & \multicolumn{6}{|c|}{ (In percent of GDP) } \\
\hline Current account & -5.1 & -6.8 & -11.1 & -5.6 & -9.4 & -7.8 \\
\hline Central government gross debt $1 /$ & 48.0 & 48.8 & 42.9 & 39.0 & 37.6 & 40.9 \\
\hline
\end{tabular}

fostering market

institutions to replace them. The result was disappointing growth, persistently high unemployment, and a widening external current account deficit (Text Table 1, Figures 1 and 2).

\section{Against this background, and in line with the recent Ex Post Assessment (EPA)} the authorities have asked the Fund to support an ambitious program of structural reforms. The EPA had presented two options for future Fund engagement: a 3-year Extended Arrangement (EA) to support comprehensive structural reforms or, if the authorities were not ready for fundamental structural reforms, a shorter Stand-By Arrangement (SBA) to support a change in the exchange rate regime. The authorities came out forcefully in favor of the first option. Staff agreed that this ambitious approach was appropriate, and noted that the successful completion of the 2003-04 SBA was a good point of departure for a new arrangement. Since the new program will become precautionary, however, staff noted that it would have to be supported by a 3-year SBA instead of an EA.

\section{The Political ANd ECONOMIC BaCkground}

\section{Political developments have cast a shadow on the business climate throughout} the post-independence period. The 2001 security crisis, though short-lived, gave rise to an enduring perception of instability since it was seen in the context of severe ethnic crises elsewhere in the region. Some more recent developments have had an offsetting stabilizing effect. In particular, the local elections in March 2005 marked progress in implementing the Framework Agreement (FA). In addition, recent international initiatives (the applications for NATO membership and European Union (EU) candidacy) and also key reforms supported by the program (notably the judiciary reform and the labor market reform) appear to enjoy support across ethnic and political divides. Consequently the next parliamentary election is not expected to derail these reforms. Broader political uncertainty persists, however, in part linked to the still unresolved status of Kosovo. 


\section{Structural impediments to economic activity and investment have been as} important as political concerns. Most strikingly:

- $\quad$ Restrictive labor market regulations created entitlements for labor without regard to the costs imposed on employers;

- $\quad$ Burdensome regulations were administered by inefficient and at times capricious state institutions;

- Inefficient and politicized courts, with little experience in commercial law, undermined confidence that contracts and property rights would be upheld in a predictable manner.

These institutional weaknesses harmed competitiveness directly, by increasing costs, or indirectly, by making FYR Macedonia an unattractive destination for investment, particularly in labor intensive sectors. Most affected were foreign investors, who found it particularly difficult to operate in this environment, and SMEs who had only limited capacity to absorb the costs of compliance. By increasing non-wage costs of employment, structural impediments also contributed to the very high rate of measured unemployment. ${ }^{1}$ And, finally, weak institutions contributed to the emergence of a large grey economy where employers and employees simultaneously avoid taxes, regulation, and rigid labor laws.

5. The effects on economic performance have been clearly discernable. Quantitative indices of competitiveness show clear signs of

Text Chart 1. Trend in Exports, 1998-2005 (In million Euro)

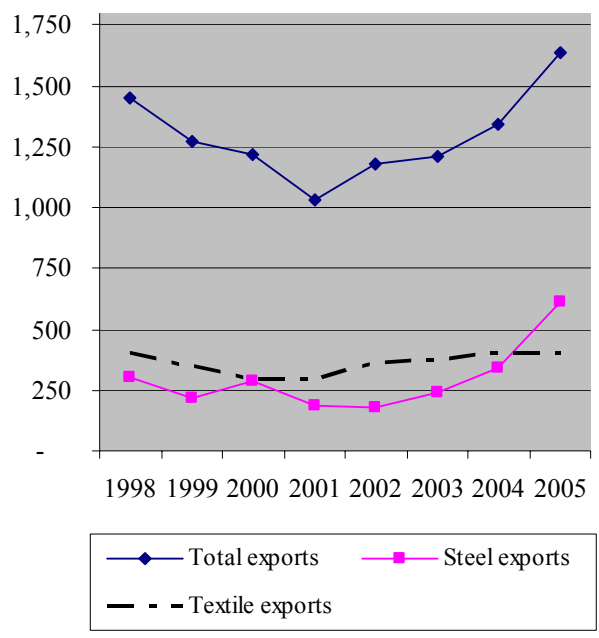
weakness (Box 1) which is also reflected in macroeconomic performance: slow growth, high measured unemployment, and a persistently wide trade deficit (Figure 2, Table 1). In addition, reflecting the low levels of FDI, the export base is still narrow: indeed, the large swings in the external current account - and growth — during the last 10 years are symptomatic of a continued dependence on exports of metals and textiles (Text Chart 1).

\section{The devaluation of $\mathbf{1 9 9 7}$ made little} dent on macroeconomic performance. The data show no discernable impact on merchandise trade (Box 1), suggesting that the

\footnotetext{
${ }^{1}$ Unemployment data, as well as data on wages, employment and labor force participation, is too weak to support a quantitative analysis of labor market conditions.
} 


\section{Box 1. FYR Macedonia: External Competitiveness}

Since the source data for ULC-based REER calculations is exceptionally weak, inferences about competitiveness need to be drawn from indirect indicators. In particular, market penetration by FYR Macedonian exporters is still lower than in 1996 (Figure A). Furthermore, a cross-country comparison suggests that gross wages in FYR Macedonia are high relative to the norm for countries with a similar GDP per capita (Figure B).

The benefits of the July 1997 devaluation were small and short-lived. The devaluation led to a sharp depreciation of the REER (ULC-based) but this was reversed over the next few years (Figure C). There was little impact on merchandise trade, however, and export share continued to decline (Figures A and D).

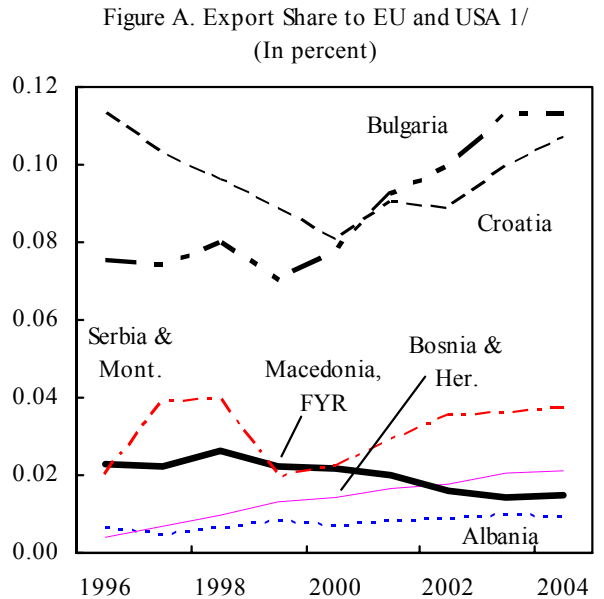

Figure C: Real Effective Exchange Rate (June 1997=100)

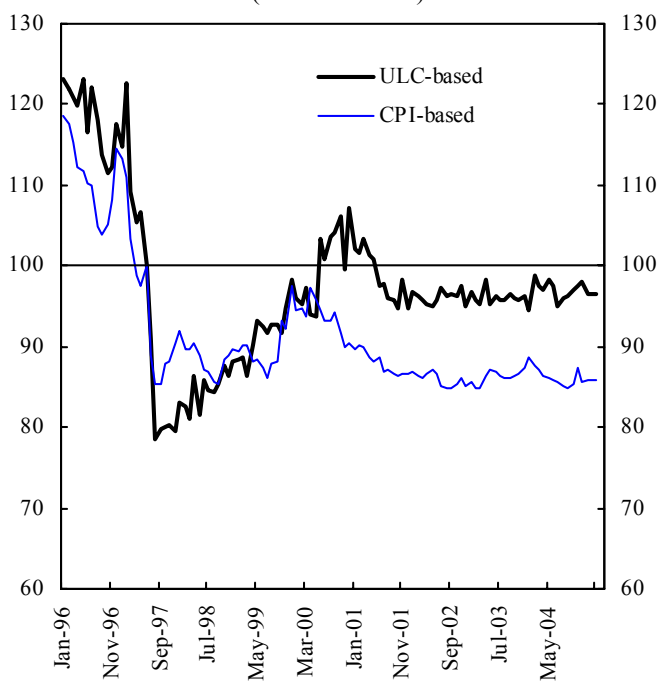

Figure B: Selected Transition Countries: Gross

Wages and Productivity, 2004

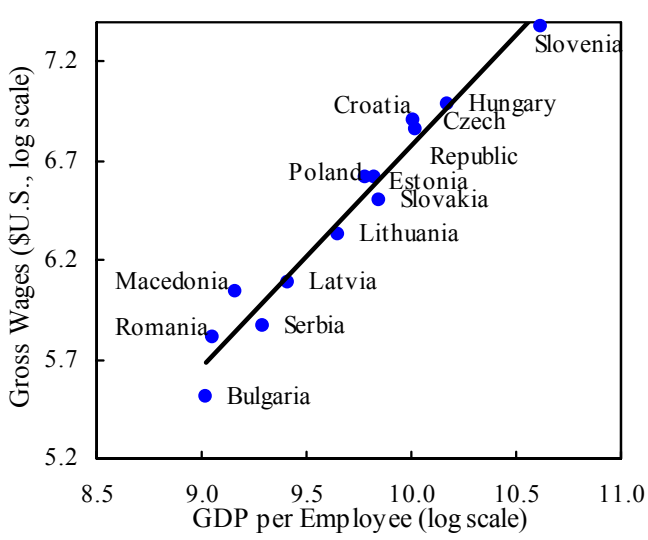

Figure D. Exports and Imports (In million Euro, deflated by the Euro zone price index, 1994=1)

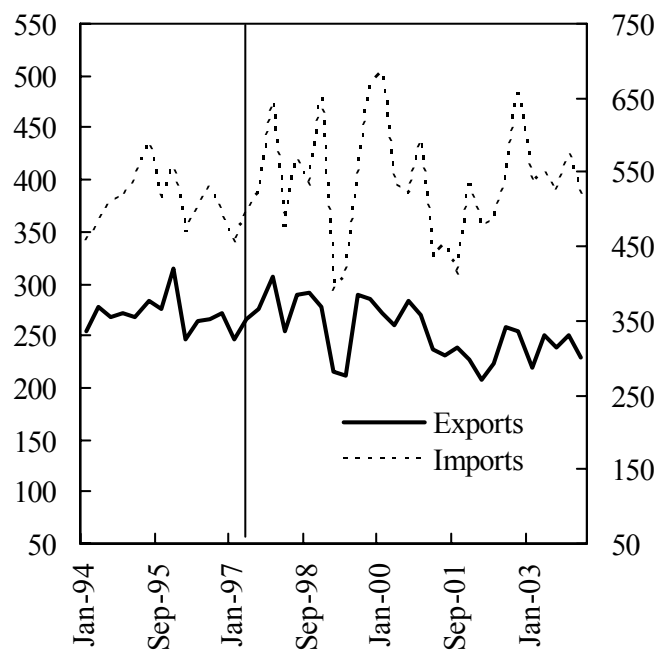

1/ Exports of a country to the US and the EU as share of world exports to the US and the EU. 
supply response was weak because of structural impediments and that producers found that their cost gains were quickly eroded by the gap in productivity growth vis-à-vis foreign producers. Looking forward, the lesson from this episode is that it is essential to approach the competitiveness problem by addressing the structural rigidities that are its root cause.

\section{Turning to macroeconomic developments, the fiscal stance has been tight-a} balanced budget in 2003 and a surplus in 2004-but there are underlying pressures on spending, revenue and financing. On expenditures, government investment is likely to increase from the very low levels of recent years as the authorities improve their implementation capacity (Table 2). Other expenditures also present fiscal risks: there is constant pressure to raise salaries; commitments under the FA may turn out more costly than expected; and, if FYR Macedonia becomes a candidate for EU accession, the need to cofinance grant-supported projects may impose an additional burden. The revenue side also raises concerns: grants in support of the FA are drying up; customs tariffs are declining in line with existing commitments to the WTO (Text Chart 2); and the new, funded, pension scheme will divert contributions away from the existing pay-as-you-go scheme. Finally, the capacity to finance the deficit has increasingly come to constrain fiscal policy options. Declining official budget financing and rising amortization payments (Text Chart 3) are making FYR Macedonia increasingly dependent on market finance at a time when the domestic treasury bill market is still undeveloped, and the capacity to tap international markets is untested.

Text Chart 2. Tax Revenues as a Percent of GDP

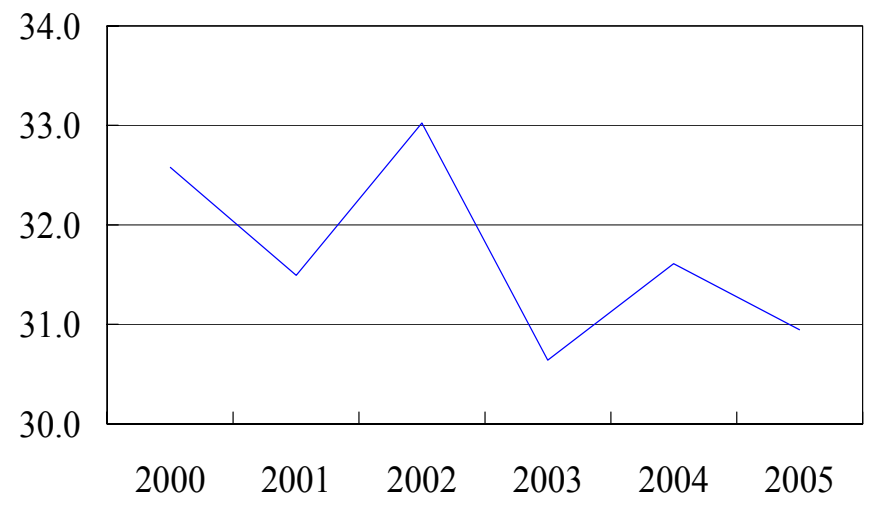

Text Chart 3. Amortization, Budget Support and Net Issuance of T-Bills

(In millions of denars)

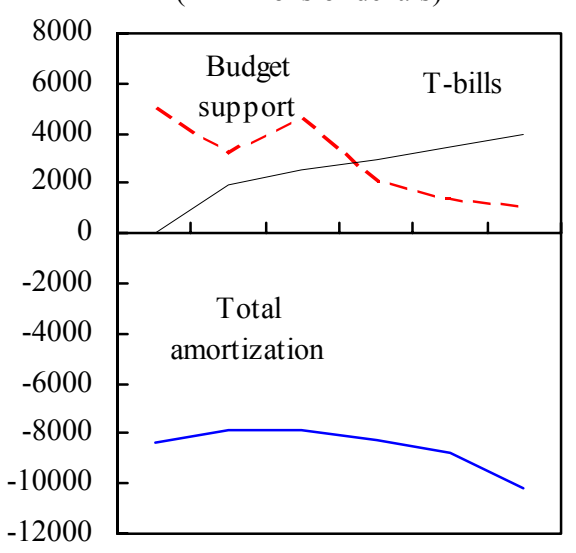

200320042005200620072008 
8. High and rising imports and the still-undeveloped financial sector have made it difficult to maintain reserve cover. The difficulty stems from the interaction between a high import ratio and a low rate of monetization (Text Chart 4). Taken together, these

Text Chart 4. Ratio Broad Money to Imports

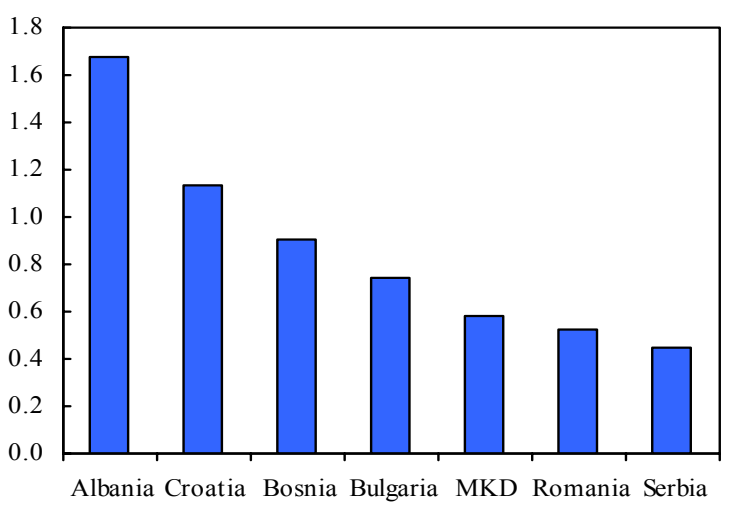

features imply that the central bank purchases of foreign exchange needed to preserve import cover represent a very large share of banks' financial resources. Consequently the authorities have not been able to increase international reserves by enough to keep up with imports even when interest rates have been high and budget has been balanced or in surplus. Therefore import cover is now well below the peak reached after the privatization of telecommunications in 2001 (Figure 4).

9. The rapid expansion of bank credit, now in its third year, reflects a structural shift to more commercial bank intermediation (Box 2). Neither the increases in interest rate on NBRM bills since late-2003 nor the still high lending rate, have significantly dampened the credit boom, partly because much of the new lending is in, or indexed to, foreign currency. While the boom has raised credit risk, including from unhedged foreign exchange exposures by borrowers, the quality of banks' loan portfolios has improved and stress tests suggest that balance sheet risks remain small (Figure 3, Table 4 and 11).

10. Private sector external vulnerabilities are also still low. The scope for speculative attacks is limited because nonresidents hold negligible amounts of denar assets and the NBRM's administrative requirements discourage short term inflows. Moreover, the NBRM's foreign asset position easily covers all denar deposit holdings. Balance sheet risks are further reduced by widespread natural hedges which include large inflows of remittances to households and significant price indexation. But rising international interest in FYR Macedonian financial instruments - following last year's positive sovereign ratingand a continuation of the lending boom could increase vulnerabilities. ${ }^{2}$

\footnotetext{
${ }^{2}$ FYR Macedonia's first sovereign credit rating by Standard and Poor's was one notch below investment grade.
} 


\section{Box 2. Credit Growth}

Credit growth accelerated in 2002, triggered by a decline in the interest rate on central bank bills and by more aggressive lending to households due to growing competition among banks. Salient features include:

- The size and speed of the credit boom are in line with other countries in the region (see Figures A and B).

- Euroization of the credit stock increased, partly because of a move towards longer-term loans which are almost all in foreign currency (Figure C).

- In 2002 banks started to offer new loan products to households whose share of new lending rose sharply (Figure C).

Figure B. Private Sector Credit Growth (2003-04 eop; in percent)

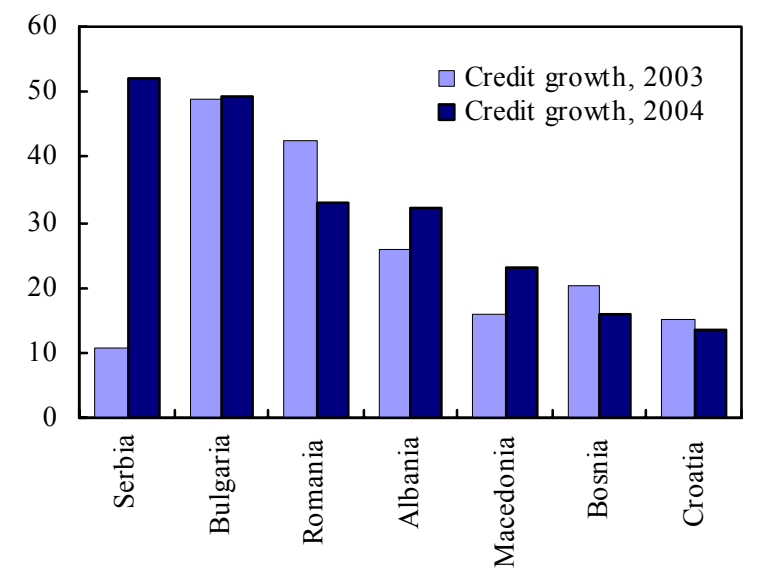

Figure A. Private Sector Credit, 2004 (In percent of GDP)

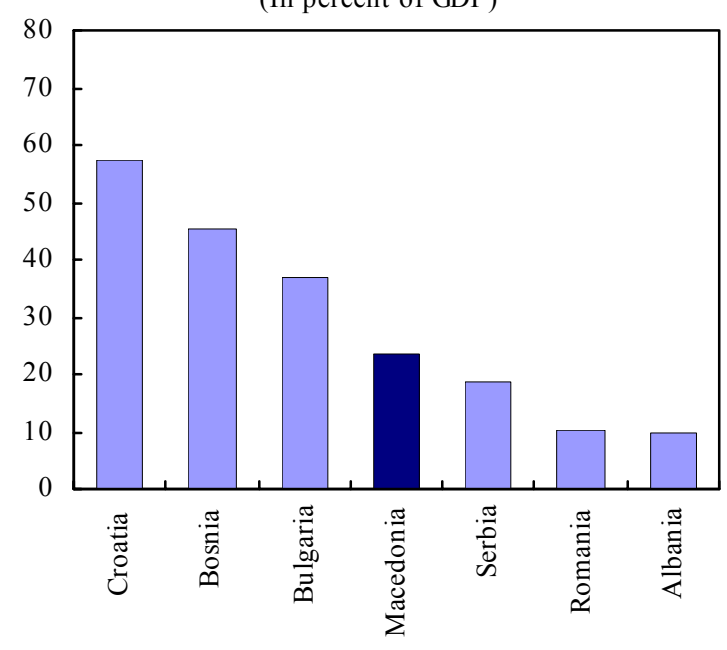

Figure C. Foreign Currency and Household Credit

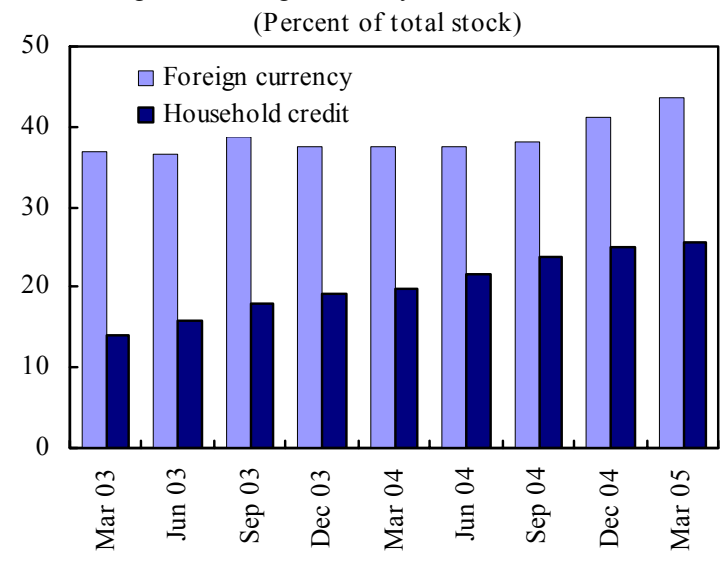




\section{The Program}

\section{A. Macroeconomic Framework}

\section{The objectives of the proposed three-year economic program are to increase} growth and employment, reduce the external imbalance, and safeguard past gains in macroeconomic stabilization. The program relies on structural reforms to strengthen competitiveness. Based on the 1997 experience, the supply response to devaluation would likely be small and transitory because of the continued presence of structural rigidities, and the risk of high pass-through in this open and increasingly euroized economy. The core of the program is therefore ambitious reforms - notably of the labor market and the courts - aimed at raising productivity growth, fostering entrepreneurial activity and improving the functioning of the labor market. At the same time, macroeconomic stability will be promoted by fiscal restraint, higher international reserve cover, and an overhaul of fiscal and monetary institutions. Since the effects of structural reforms on growth and the balance of payments are highly uncertain in both magnitude and timing, the need for additional policy measures will be kept under review.

12. The policies under the program are expected to increase economic growth while reducing the external current account imbalance. Staff and the authorities discussed a

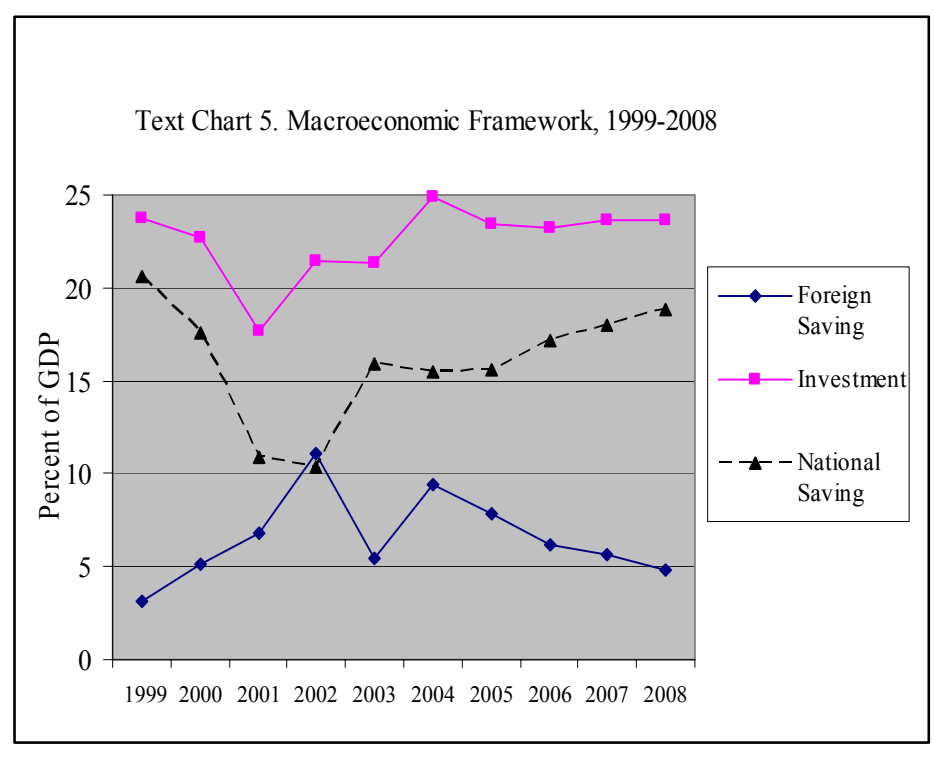
medium term projection in which GDP growth accelerates from 2.9 percent in 2004 to about 4.5 percent by 2007 and the current account deficit shrinks from 9.4 percent of GDP in 2004 to 4.8 percent in 2008 (Text Chart 5 , Figure 5 and Table 6 ). The improved business climate is expected to sustain investment at nearly 25 percent of GDP. With foreign saving declining, the investment will increasingly be financed by domestic saving, which is projected to return to its pre-2001 level.

\section{The external debt sustainability analysis (DSA) underscores the need for} structural reforms (Appendix III). While the baseline scenario and the standard bound tests suggest only limited vulnerabilities, the scenario based on historical averages results in worrying debt dynamics. Against this background, a turnaround of the historic trends following the reforms included in this program is essential to ensure long-run external sustainability. 
14. In order to protect the economy against external shocks, privatization proceeds will be set aside to boost reserves. Proceeds from privatizating the electricity and telecoms utilities will be used to raise gross international reserves to 4 months of imports by the end of the program. This target, which is appropriate given the fixed exchange rate, the narrow export base, and the still limited access to international capital markets, would have been difficult to reach otherwise. After the program has ended, the smaller current account deficit, combined with the deepening of monetization and small fiscal deficits, should allow the NBRM to maintain reserve cover at this level. In addition to making it possible to reach an appropriate reserve level, the privatizations will eliminate the conflict of interest arising from the government's present dual role as shareholder and regulator.

\section{B. Fiscal Policy}

15. The program builds on the successful fiscal adjustment under the previous SBA by targeting deficits that will reduce the government's debt ratio (Text Chart 6 and 7). The medium term target for the consolidated central government deficit is 0.6 percent of GDP. This tight stance respects financing constraints and positions the budget for uncertain future expenditures. The fiscal debt sustainability analysis shows that government debt dynamics are generally favorable (Appendix III). The lower government debt ratios, combined with the modest deficits will help secure the government's ability to tap private credit markets in the coming years.
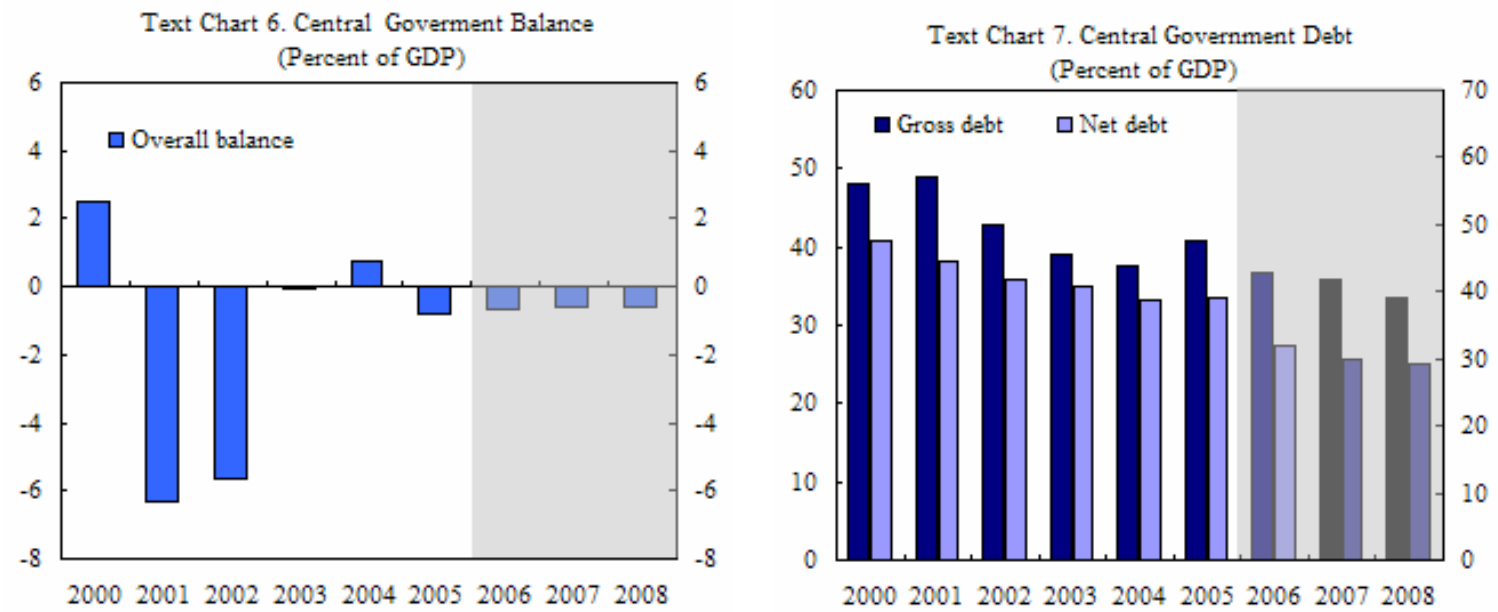

16. For 2005, the authorities have passed a supplementary budget with a deficit (0.4 percent of GDP after correcting for central bank recapitalization) that is in line with the medium term fiscal program (prior action). While this represents a significant tightening relative to the original budget deficit target for the year (1.2 percent of GDP) the authorities agreed that this was appropriate in light of the unexpectedly large 2004 current account deficit (8 percent of GDP after correcting for exceptional factors), and the need to avoid a large fiscal impulse relative to the 2004 fiscal surplus. 
17. A successful transition to market financing will require not only fiscal prudence but also the development of a debt management strategy. The Ministry of Finance has therefore established (with Fund technical assistance) a Public Debt Management Department. The authorities' initial strategy focuses on developing the domestic securities market (see $\$ 27$ below) and on entering the Eurobond market with a modest first issue in late 2005. The Eurobond issue is part of a broader restructuring that will extend the average maturity and lower the average interest rate on government debt.

\section{The key to reaching the program's ambitious deficit targets is a broad-based} containment of expenditure. The authorities have committed to keep the government wage bill from rising faster than total tax revenues during the program period. In addition, they will complete functional analyses of line ministries which will enable the government to better define spending priorities. The wage ceiling is an indicative target for the program; completing the functional analyses is a structural benchmark for the first review. Further measures will be agreed when the 2006 budget is discussed in the fall.

\section{In the area of taxation, the authorities resisted proposals to change tax policy}

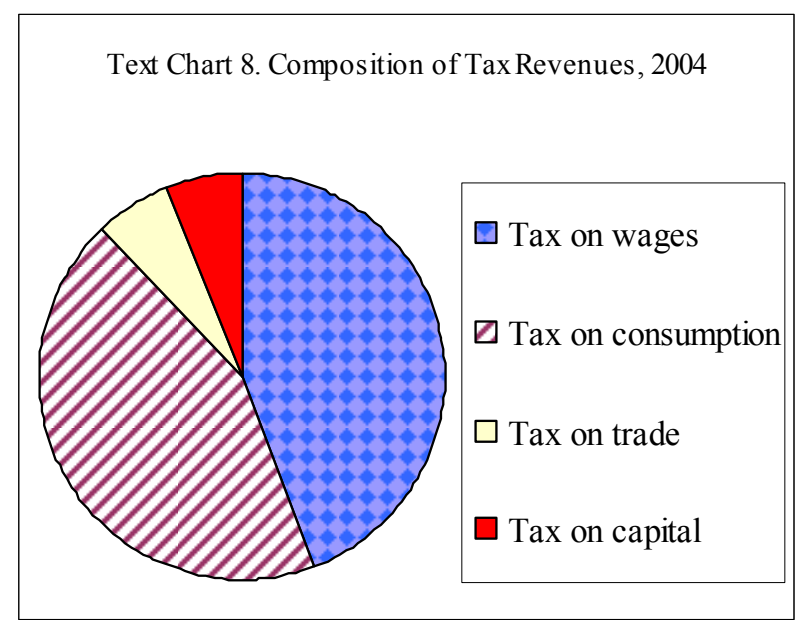
but have decided to launch an ambitious set of reforms of tax administration. On tax policy, Fund staff urged the authorities to consider reforms that would stem the projected secular decline in revenue while shifting the tax burden away from wages, which are heavily taxed (see Text Chart 8). ${ }^{3}$ Staff also urged the authorities to include non-wage compensation in taxable income. The authorities resisted both proposals on the grounds that they would be unpopular and difficult to explain in the run-up to elections. They have thus opted for limited tax policy changes at this stage:

broadening the base of the Corporate Income Tax and putting a moratorium on new free economic zones (MEFP, 34). On tax administration, however, the authorities have more ambitious plans (MEFP, 35). Organizational changes to promote better registration, collection, audit and enforcement and to provide equal treatment for taxpayers are already underway. In addition, to strengthen the PRO over the medium term, the authorities will enhance headquarters capacity (performance criterion). While acknowledging the PRO's reform efforts, staff will continue to press for tax policy reform.

\footnotetext{
${ }^{3}$ The authorities' projections appropriately reflect declining grants and customs revenues and make no allowance for gains from administrative measures. Even after correcting for these factors, however, the staff views the authorities' projections as very cautious.
} 


\section{The authorities are also planning an ambitious multi-year program to} consolidate and rationalize the collection of social insurance contributions and personal income taxes (MEFP, 36). Currently, the contributions and PIT are collected by two different social funds and the PRO. This inefficient use of scarce administrative resources imposes a heavy administrative workload on employers. Drawing on technical assistance from FAD, the authorities have developed a strategy that will eventually merge all collection at the PRO. Since the reform requires cooperation by several agencies, the government has created a high level inter-ministerial committee to manage the reform (prior action).

21. Although progress has already been made in budget planning and execution, the program includes further improvements in these areas, with an emphasis on fiscal transparency and budget execution (MEFP, 32). Several legislative initiatives, which will take effect in 2005, aim at smoothing budget execution, whose unpredictable movements have complicated macroeconomic management in recent years. The transparency of budget preparation and planning will be enhanced — starting with the 2006 budget — by using a medium-term fiscal framework to assess medium-term fiscal policies. To further improve fiscal transparency, the Ministry of Finance will continue to move toward GFS-consistent definitions in fiscal planning and reporting (structural benchmarks).

\section{The implementation of health sector reform is a litmus test for the authorities'} commitment to fight corruption (MEFP, 37). The reform plan-which was prepared in consultation with the World Bank-focuses on features of the health care system that have stood in the way of previous reforms: conflict of interest and a lack of accountability at the Health Insurance Fund (HIF); weak financial controls and a lack of transparency at the Public Health Institutions (PHIs). Amended legislation will increase the influence of the Ministries of Health and Finance over the HIF board, tighten conflict of interest rules for board membership, and impose clearly defined budgeting, reporting and auditing requirements on both the HIF and the PHIs. Given that health accounts for 15 percent of budget spending and given the poor track record of reform in this area, government approval of the needed legislation is a prior action for the arrangement and key steps in implementing the reform have been incorporated into conditionality (one performance criterion and two structural benchmarks). In light of the HIF's large and persistent arrears, the program also includes a ceiling on the further accumulation of arrears (performance criterion) and a commitment to inventory and audit arrears by mid-2006, and make allowance for their repayment over 2007-08 (MEFP, 38).

\section{Monetary Policy and the Exchange Rate Regime}

23. The authorities considered, but rejected, the option of moving to a more flexible exchange rate arrangement on the grounds that the current regime has, on balance, served the country well. Discussion of the exchange rate regime focused on balance sheet risks. The authorities recognized that some flexibility would sharpen banks' and borrowers' incentive to hedge foreign currency exposures but they viewed the arguments in favor of the peg as more persuasive. In particular, given the still unstable economic environment, they 
saw merit in retaining a clearly defined monetary anchor. In examining the alternatives, they took the view that inflation targeting or monetary aggregate targeting would be unworkable given the unpredictability of the monetary transmission mechanism. In contrast, limited flexibility - a narrow band — could be manageable. But this would have too small an effect on banks' and borrowers' behavior to justify the risks associated with departing from the existing well-functioning anchor.

\section{The program thus includes measures to reduce balance sheet risk and improve} the management of monetary policy under the existing peg. First, as discussed above, the peg will be buttressed by building reserves to a sufficient level. Second, the strengthened reporting on foreign exchange risks introduced under the last program will be complemented by intensified supervision (see below). And finally, with support from MFD, the NBRM has developed a program to improve the institutional framework for monetary policy, in particular, data analysis, market development, and monetary instruments.

\section{On credit growth, staff and the authorities agreed that increased bank} intermediation was a positive development, but that the transitional risks needed to be managed carefully. Since high interest rates had done little to limit credit expansion, it was agreed that attention should be focused on strengthening bank supervision in order to protect the quality of banks' loan portfolios. The NBRM already requires banks to collect data on borrowers' foreign exchange risk exposure and has made the assessment of these risks part of its regular supervision activities. As a next step, the information collected will be used to strengthen prudential regulations, especially for foreign exchange loans. New prudential regulations will be incorporated into program conditionality at the first program review, taking into account the findings of a MFD mission in October 2005.

26. Further measures to strengthen the banking sector focus on governance. The authorities will amend the Law on Banks to give the NBRM authority to enforce fit and proper requirements on existing bank shareholders (currently that authority extends only to prospective buyers) and to strengthen procedures for bank bankruptcy. The strengthening of the NBRM's legal authority is welcome but needs to be backed up by transparent procedures, clear guidelines for action, and a readiness to use the available legal tools decisively. The preparation (with World Bank support) of a development plan for risk-based supervision will be a step in this direction and is a structural benchmark under the program.

27. The development of the treasury securities market is a core priority for the program since it affects the government's capacity to borrow, the scope for central bank open market operations and the investment opportunities available for firms, households and banks. The reform has two main elements. First, the NBRM and the Ministry of Finance are helping foster the growth of a secondary market for treasuries and the creation of a repo market (MEFP, 23). More secondary trading will increase liquidity, lower the cost of borrowing and allow the government to extend maturities. Second, by the end of the year, the government and the NBRM will agree on a plan that will allow the NBRM to phase out central bank bills and use treasury securities (and repos) for open market operations (structural benchmark; MEFP, 24). This will end the segmentation into two small 
markets and consolidate all activity in a single, larger and more liquid market. It will also provide the central bank with a wider menu of options: a longer term instrument to withdraw structural excess liquidity, and shorter term instruments for day-to-day open market operations.

28. The government will bolster the NBRM's independence by increasing its capital base and establishing rules that will help it maintain a financial cushion over the medium term (MEFP, 22). The immediate need for a financial injection was created when a NBRM guarantee to a private bank was called, reducing the central bank's capital below the statutory minimum. ${ }^{4}$ In addition, the NBRM's profitability has been reduced by high interest payments on bills issued to absorb excess liquidity (see $\$ 8$ above). The undercapitalization has on occasion made central bank profitability a factor in interest rate decisions, at the expense of monetary policy objectives. The authorities have therefore agreed to a package that reestablishes the NBRM's capital base and improves its profitability.

\section{Structural Reforms}

\section{Labor market reform}

29. The centerpiece of the authorities' reform program is the recently-adopted Law on Labor Relations (LOLR) which marks a turning point in labor relations in FYR Macedonia. The old law supported a framework which awarded generous entitlements to the employee and placed a web of heavy financial and administrative obligations on the employer. Firms were bound by collective agreements signed by weak employers' associations with mandatory universal membership; the dismissal of employees was subject to complex procedures and vulnerable to court challenge; the legal framework for short term employment contracts, part time contracts and overtime was restrictive; and mandated allowances were burdensome. These features swelled the cost of production and fostered the growth of informal labor markets where workers' rights were not protected, wage arrears were endemic, and taxes were not paid.

30. The new law, which eliminates the most burdensome features of the old law, is expected, over time, to have a large impact on growth, foreign investment and the size of the grey economy. The law, which incorporates advice from both Bank and Fund staff, will make it possible to reduce non-wage labor costs and will allocate labor more efficiently. The more business-friendly environment will also encourage increased investment, particularly by domestic and foreign SMEs. And, by striking a better balance between workers' rights and employer's needs, this law, combined with the greater capacity and increased enforcement powers of the tax authorities, will create an opportunity to make a significant dent in the grey economy. This, in turn, should make it possible to reduce the tax

\footnotetext{
${ }^{4}$ The guarantee was issued in 1998 at the request of the government. The practice of providing central bank guarantees was ended in 2003.
} 
wedge on labor, bringing further improvements in competitiveness and growth. Consequently, the enactment of the LOLR was a prior action for Board consideration of the arrangement.

\section{Judicial Reform}

31. The authorities have launched an ambitious and comprehensive judicial reform program, which aims to strengthen the rule of law by conforming the court system to European standards. The strategy, which will take several years to implement, was designed with extensive technical assistance by donors (including EU institutions and member countries, NATO, and USAID), and following wide consultation among domestic stakeholders. ${ }^{5}$ The reform aims to increase the independence and professionalism of judges, eliminate court delays, remove misdemeanors and administrative cases from the regular courts, and strengthen enforcement (MEFP, 15-19). The first round of reforms consists of legislative actions to create a legal and constitutional basis for the new court system. The second round consists of administrative reforms: training for judges, improved court administration and budgeting, and the creation of a new enforcement agency.

\section{Staff and the authorities agreed that the judicial reform will have a major} impact on the business climate. Indeed, lengthy court delays, capricious and politicized

\begin{tabular}{|lccc|}
\hline \multicolumn{4}{|c|}{ Text Table 2. Contract Enforcement Efficiency, 2004 } \\
\hline & FYR Macedonia & Regional average & OECD average \\
\hline & & & \\
Number of procedures & 27 & 29 & 19 \\
Time (in days) & 509 & 412 & 229 \\
Cost & 32.8 & 17.6 & 10.7 \\
(in percent of debt) & & & \\
\hline Source: World Bank & & & \\
\hline
\end{tabular}

judgments and poor enforcement, have been major deterrents to formal commercial activity and investment (Text Table 2). In addition to eliminating these deterrents, the reform will strengthen public institutions such as the

NBRM and the PRO by giving them the authority to levy administrative sanctions without prior court approval (structural benchmark).

\section{The legislative component of the judicial reform program is on track, but} implementation could falter for lack of attention to institution-building. The mission emphasized that it would be important that the reform go beyond procedural issues and address also institutional aspects; the specialization of judges being an important example. In particular, the mission urged the authorities to prepare an analysis of the financial and human resource requirements of the reform - and of the proposed new court system - and to ensure that the court budget office is provided with appropriately qualified staff. More broadly, and in concert with other donors, the mission recommended to appoint a project manager with

${ }^{5}$ The Fund mission did not play an active role in designing the reform (which falls outside of the expertise of Fund staff) but did discuss the impact of the reform on the business climate. 
experience in implementing administrative reforms. The authorities agreed to address the staff's concerns but they did not take on board the recommendations regarding court budgeting and they have not appointed an appropriately qualified project manager. The implementation of the judicial reform will therefore need to be monitored closely in the context of program reviews.

\section{Other}

\section{The labor market and judicial reforms will be complemented by a number of measures to strengthen the framework for business activity (MEFP, 21).}

- $\quad$ A consolidated procedure for company registration will be put in place by end-2005. This long delayed reform will shorten the time needed to open a business, which currently far exceeds the regional average (Text Table 3).

- $\quad$ The Word Bank supported FIAS project will address the excessive number of licensing requirements.

- $\quad$ Also with support from the World Bank, the government plans a project to improve legislative procedures and the institutional framework for corporate governance.

- $\quad$ Other reforms include measures to strengthen the institutional framework for bankruptcy and measures to improve land registration in order to make land useable as collateral.

These projects are expected to take several years to come to fruition.

\begin{tabular}{|lccc|}
\hline \multicolumn{4}{|c|}{ Text Table 3. Procedures for Starting a Business, 2004 } \\
\hline & FYR Macedonia & Regional average & OECD average \\
\hline Number of procedures & 13 & 9 & 6 \\
Time (in days) & 48 & 42 & 25 \\
Cost & 11.6 & 15.5 & 8.0 \\
(in percent of income per capita) & & \\
\hline Source: World Bank & & \\
\hline
\end{tabular}




\section{Program Modalities}

\section{A. Type of Program and Proposed Access}

\section{The authorities have requested a three-year SBA in the amount of} SDR 51.675 million (75 percent of the quota or 25 percent annually), with some frontloading of purchases. Purchases are to be phased in 13 tranches, including an initial SDR 10.5 million to be drawn on approval and 12 equal quarterly tranches of SDR 3.4 million each. The front-loading is justified by strong prior actions.

36. After making the initial purchase, the authorities intend to treat the program as precautionary. The initial purchase is needed to boost the level of gross reserves. Thereafter, privatization proceeds, foreign direct investment, and debt financing from official and market sources should be sufficient to build up, and maintain an adequate reserve cover (Table 5). Nevertheless, the undrawn amounts under the Fund arrangement will constitute a precautionary line of credit for possible balance of payments shocks.

37. The authorities have requested an extension of repurchase expectations totaling SDR 5.4 million falling due before end-September 2006. The basis of this request is external financing needs during the next twelve months and the deterioration of the external position relative to the outlook in March 2003, when the last SBA was approved. In particular, reserve cover at end-2004 was 3 months of imports, compared with 4 months projected when the arrangement was approved. Given this weaker position, the authorities' commitment to restore reserve cover to 4 months of imports has required an ambitious privatization effort and a significantly tighter fiscal stance than was originally anticipated.

38. A three-year SBA, is an appropriate instrument to support the proposed structural reforms, which will be implemented over a three year horizon. The longerterm commitment will also reduce market uncertainty about the macroeconomic framework, which has in the past put pressure on the balance of payments during periods between IMF arrangements.

\section{B. Program Conditionality and Monitoring}

39. In view of the structural character of the program, conditionality includes structural prior actions, performance criteria and benchmarks (MEFP, Table 2). The structural prior actions include passage by Parliament of a new Law on Labor Relations ( $\mid 29)$; the establishment of an interministerial steering committee for payroll tax reform ( $\mid 20)$; and government submission to Parliament of legislation related to HIF reforms ( $\$ 22)$. Structural conditionality for the first and second program reviews includes follow-up measures for the prior actions and further conditionality on tax administration, the financial sector and the health sector.

40. In addition, program implementation will be monitored using quarterly quantitative performance criteria and indicative targets (MEFP, Table 1) and 
6 semiannual reviews. The quantitative performance criteria of the program-notably the ceilings on the budget deficit and borrowing and the target on NIR accumulation - have been designed to ensure fiscal and external sustainability during and beyond the program. These performance criteria are complemented by selective indicative targets, including a ceiling on the central governments' wage bill and arrears. The quarterly quantitative targets have been set until end-2006 with the understanding that targets for latter periods will be set during subsequent program reviews.

\section{Capacity to Repay the Fund and Program Risks}

41. Should the need arise to draw on the resources available under the proposed SBA, staff is confident that FYR Macedonia could meet its obligations to the Fund. Under the proposed purchase schedule (assuming full purchases under the proposed SBA), FYR Macedonia's annual debt service to the Fund would peak in 2010 at 24.9 percent of quota (Table 9 ), equivalent to 0.7 percent of exports. Against this moderate exposure and the good track record of repayments, FYR Macedonia is not expected to encounter difficulties in meeting its obligations to the Fund.

42. Progress has been made in implementing the recommendations of the Fund's Safeguards Assessment and a follow-up assessment is planned to identify remaining weaknesses. A full Safeguards Assessment was completed on April 24, 2003 and the majority of its recommendations have been implemented by the NBRM. In line with the Fund's Safeguards Assessment policy, a follow up assessment will be performed in the context of the proposed arrangement.

43. Areas of program risk include privatization, political pressures related to the fall 2006 election, and the current account. While the authorities appear committed to the privatization of ESM and the sale of residual shares in Macedonia Telecom, the timeframe is tight since privatizations are not permitted during the 6 months prior to an election. Spending pressures, especially on wages, could increase in the run-up to the election. In addition, current account developments are subject to considerable risks regarding the size and timing of the effects of structural reforms. Finally, the narrow export base (mainly steel, textiles and agricultural products) makes the current account vulnerable to changes in these sectors.

\section{Staff Appraisal}

44. The main economic challenge facing FYR Macedonia today is to turn around a weakening business climate through structural reforms. Structural rigiditiesburdensome labor regulations, poorly functioning courts, impediments to entrepreneurial activity - have combined with political uncertainties to produce years of weak output and employment growth. They have also contributed to rising current account deficits and declining international reserve cover despite tight fiscal policies and high interest rates. Until addressed, these structural rigidities will remain the Achilles' heel of an otherwise impressive macroeconomic stabilization. 
45. The authorities' emphasis on structural reforms is therefore on the mark. The reforms are well focused on institutions - the labor market and the judiciary — which are foundations of a well-functioning market economy. Since these reforms are critical to the macroeconomic objectives of the program - increased supply-driven growth, higher levels of foreign direct investment, improved competitiveness, and a reduction of the balance of payments disequilibrium - they have been incorporated into program conditionality.

46. The labor market reform represents an important break with the past. The staff welcomes the new Law on Labor Relations, which strikes a good balance between employer's needs and workers' rights. Its provisions will increase FYR Macedonia's attractiveness to foreign investors and help draw employment out of the grey market. The result will be improved employment conditions, better tax collection, and heightened respect for the law. To ensure that these benefits flow as anticipated it will be important to strengthen, with World Bank support, the supporting institutions for enforcement and conflict resolution.

\section{A reduction of payroll taxes would be a powerful complement to labor market}

reform. While the authorities were not ready for tax policy reform at this stage the staff encourages them to keep this option under consideration. Another opportunity for reducing payroll taxes will arise when the new labor code and the strengthening of tax administration start increasing the tax base. This would also be a good occasion to widen the tax base by including non-wage compensation into taxable income.

\section{The judicial reform strategy is well designed and comprehensive; if fully} implemented it will have a transformative impact on the business climate. Efficient, professional courts with well trained judges will provide, for the first time, the legal supports needed for a functioning market economy. While some benefits of the reform should be evident immediately, its full effectiveness will require that legislative and constitutional changes be converted into changes in behavior on the ground. This will require further attention to institution building.

\section{Fiscal and monetary policy will face significant challenges during the period} before structural reforms take effect. Declining official inflows and the government's still small capacity to borrow will make it difficult to finance the deficit while increasing international reserves. The decision to keep a strict limit on the fiscal deficit is therefore critical. Two large asset sales - the ESM privatization and the sale of residual telecoms shares - will close the remaining gap. In addition, the ESM privatization will put electricity generation on a commercial basis while the sale of telecoms shares will eliminate a conflict of interest arising from the government's dual role as shareholder and regulator.

\section{The authorities' efforts to increase their borrowing capacity are also welcome.}

The planned first foray into the international sovereign debt market, which is being mounted on an appropriately cautious scale, should set the stage for future international borrowing. However, access to international markets should not reduce efforts to raise finance domestically. 
51. The three year old credit surge and high euroization suggest that balance sheet risks, though still modest, are rising and need to be addressed in a timely way. The authorities have decided to do so through intensified bank supervision rather than exchange rate flexibility. Since full flexibility could dislodge the exchange rate anchor, while limited flexibility would not have the desired effect on behavior, staff supports this decision.

Measures to strengthen bank supervision will be incorporated in the program at the first review.

52. Finally, the proposed health sector reform represents good progress on a longstalled agenda. The focus on improving governance at the HIF and financial controls at the HCIs is appropriate and will make the health sector more accountable in its use of public funds. The change will also allow the government to set objectives for health care institutions that are in line with broader national priorities.

53. In view of FYR Macedonia's external financing needs during the next twelve months, the strong program and the good record in implementing the previous SBA, the staff supports the authorities' request for a new SBA and for an extension of repurchase expectations. If fully implemented, the program will create a sound basis for access to international credit markets, set FYR Macedonia on the path to EU accession and lead to an orderly exit from IMF support. 


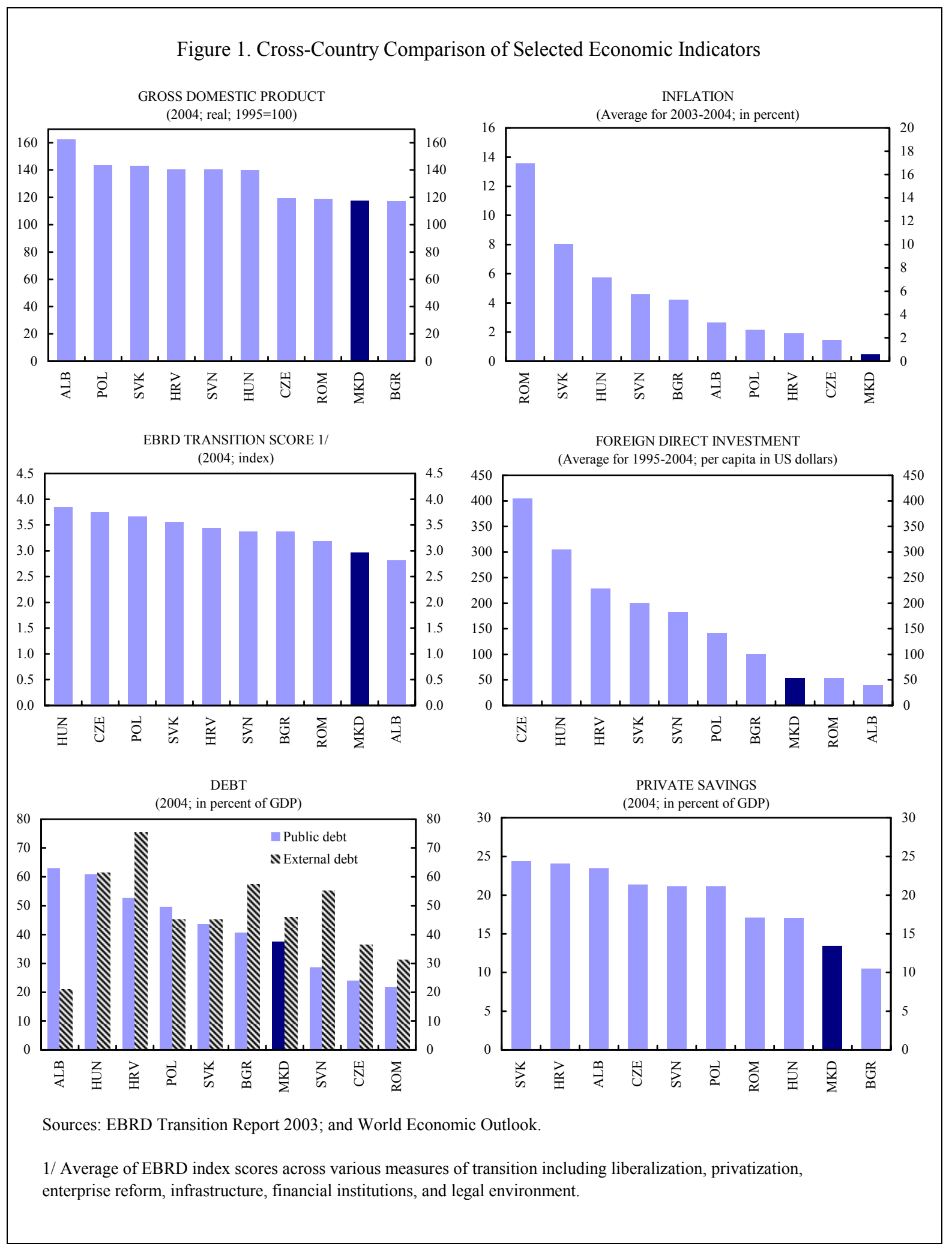


Figure 2. FYR Macedonia: Selected Economic Indicators, 2000-05 1/
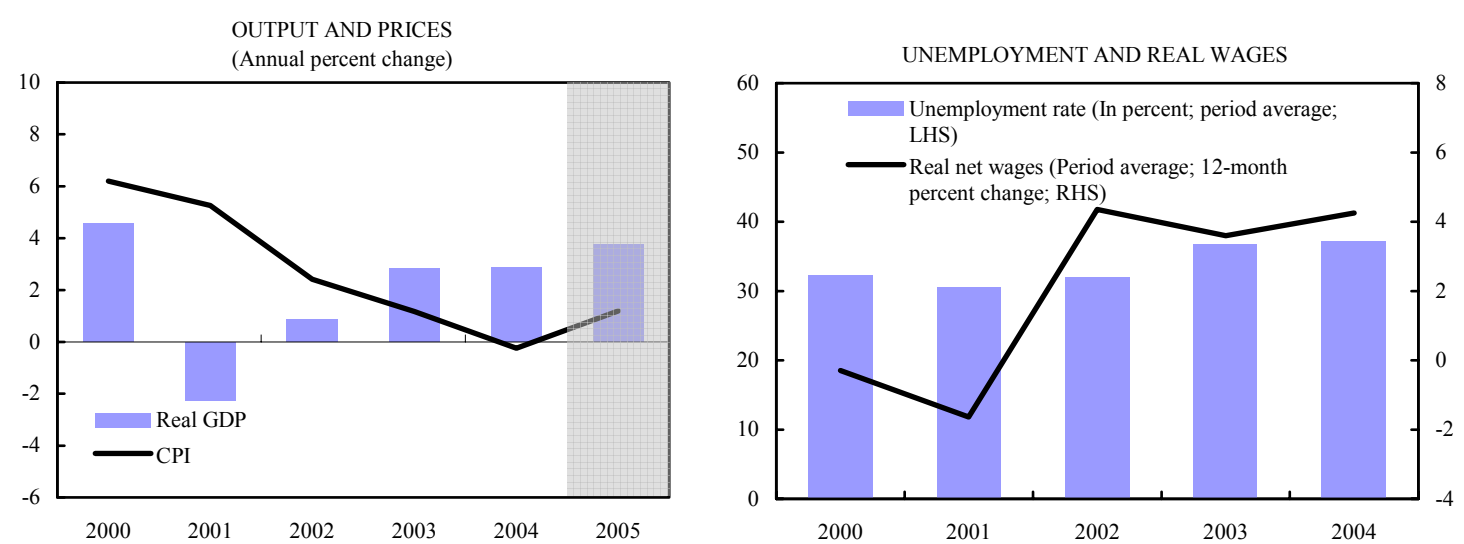

CURRENT ACCOUNT BALANCE

(In percent of GDP)
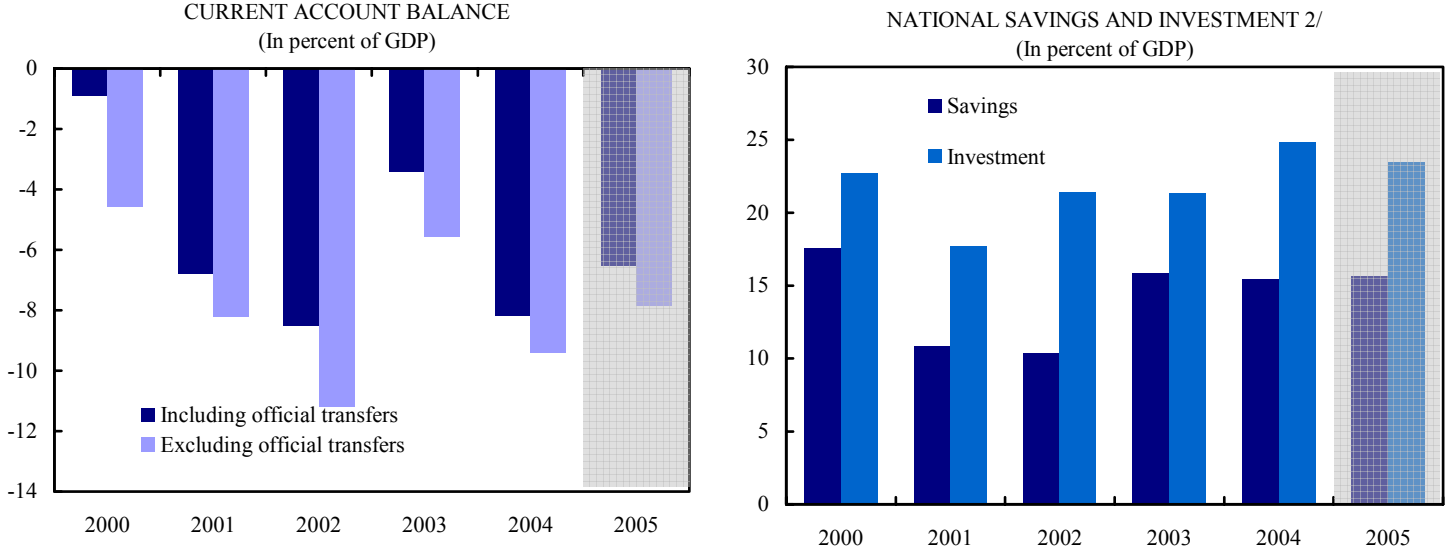

CENTRAL GOVERNMENT BUDGET

(In percent of GDP)

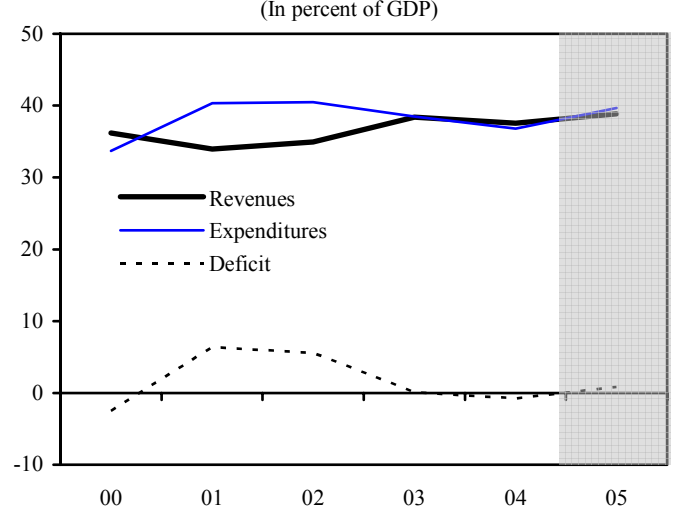

TRADE DEFICIT

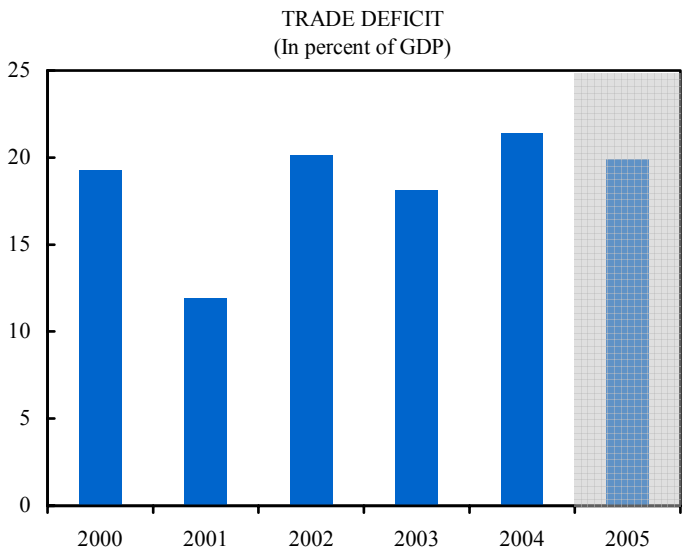

Sources: Macedonian authorities; and IMF staff estimates.

1/ 2005: Fund staff projections.

2/ 2004: Fund staff estimates. 
Figure 3. FYR Macedonia: Monetary and Financial Indicators, 2000-05

Broad money growth continued to be strong,....

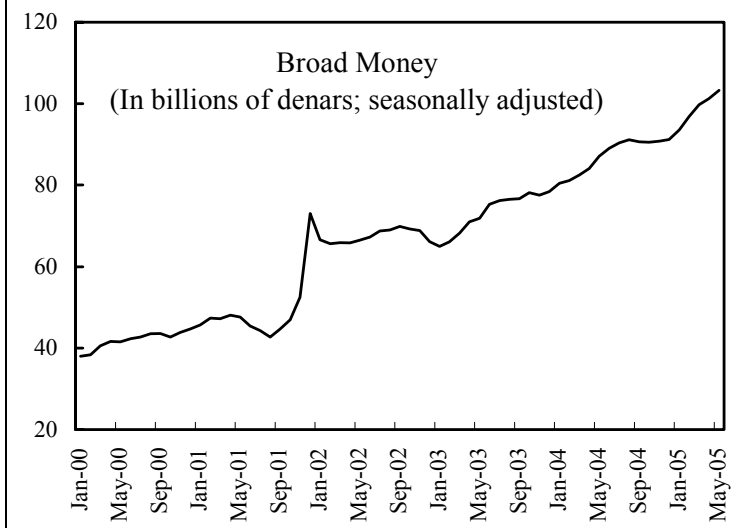

As banks invested less foreign currency deposits abroad,....

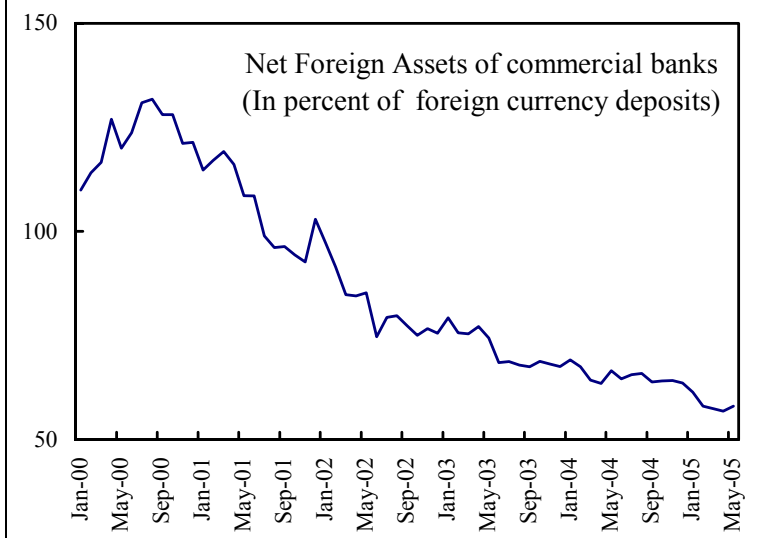

...which is also reflected in declining lending rates...

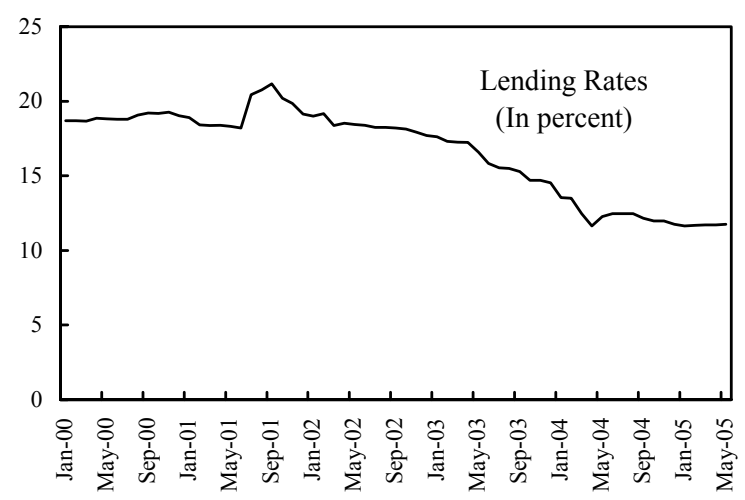

...far exceeding GDP growth, as reflected in velocity.

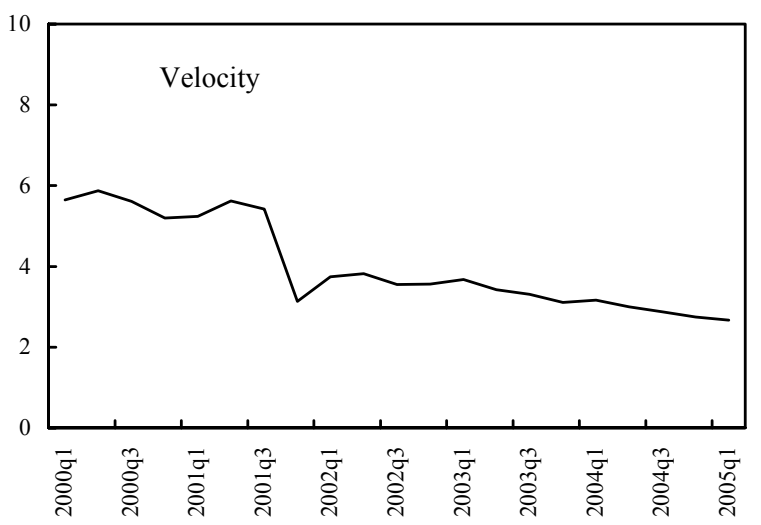

.... funding was available to fuel private credit growth...

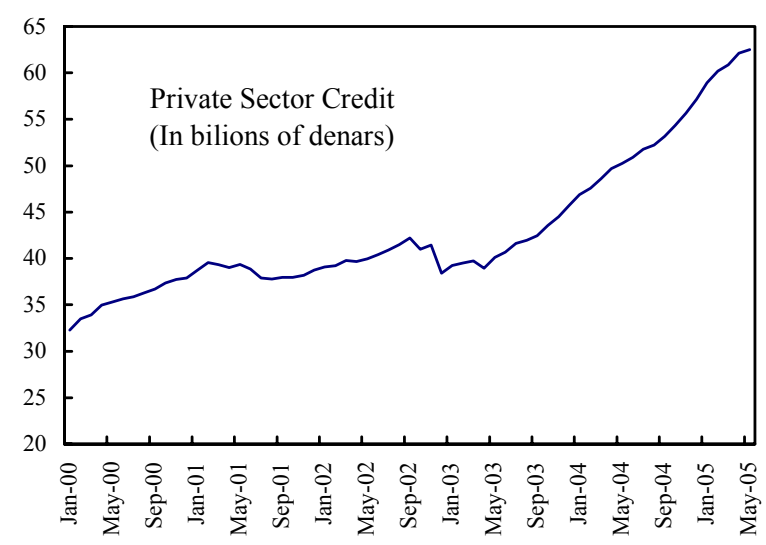

...despite the 2004 increase in the NBRM interest rate.

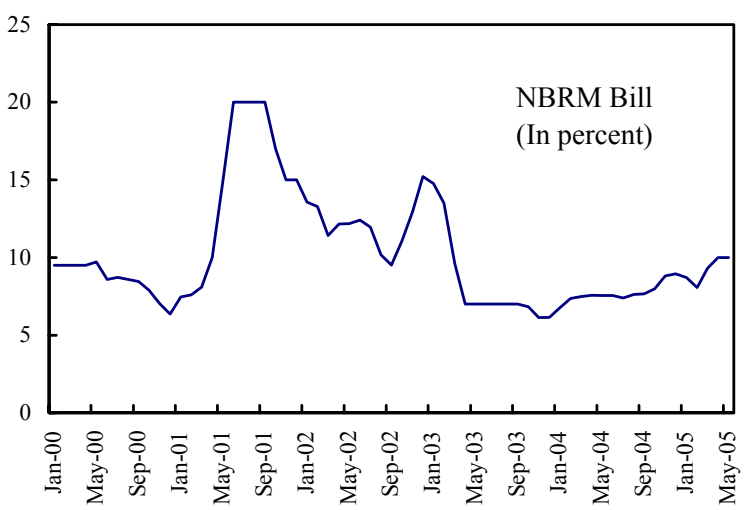

Sources: FYR Macedonian authorities; and IMF staff estimates. 
Figure 4. FYR Macedonia: External Sector Developments and Competitiveness, 2000-05

Current Account

(In percent of GDP; seasonally adjusted)

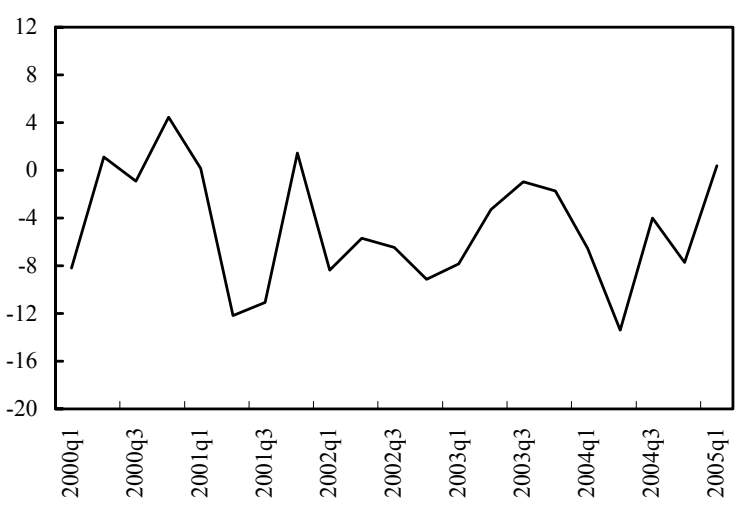

Exports 1/

(year-on-year change)

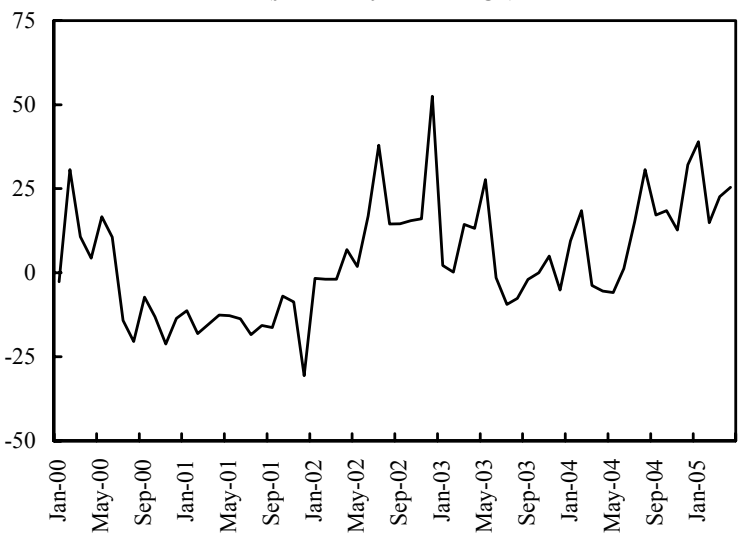

Reserve Stock

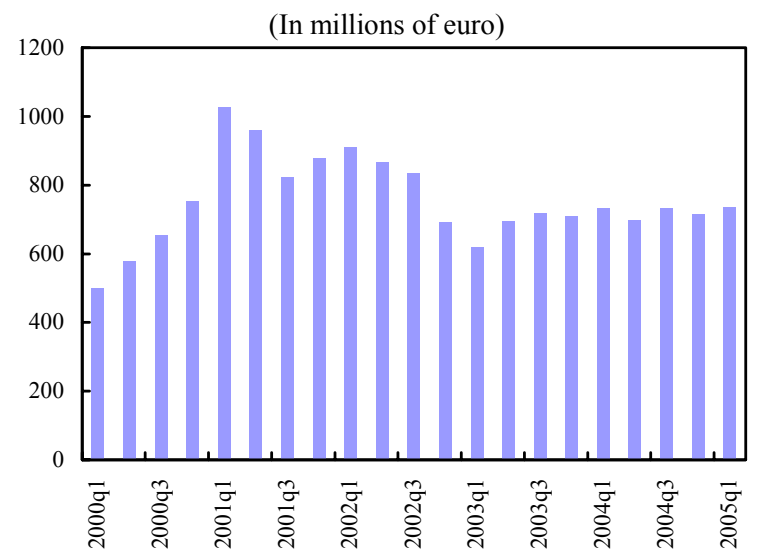

Imports, excluding oil 1/2/

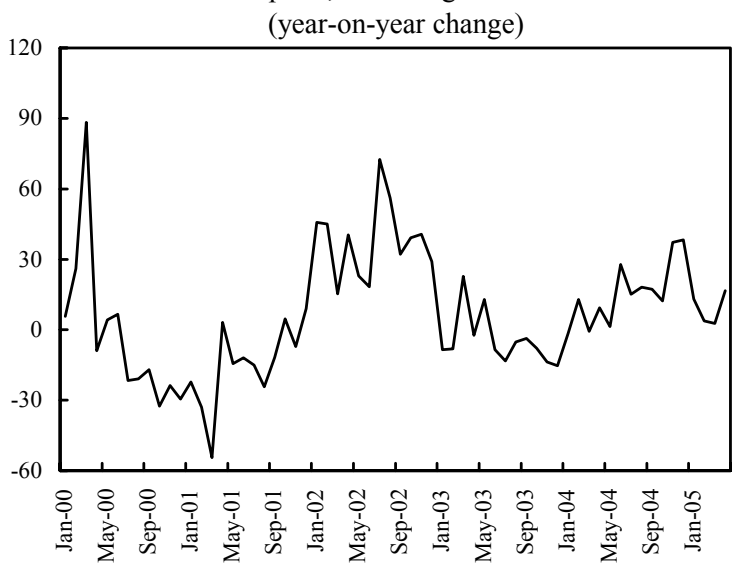

Transfers

(In percent of GDP)

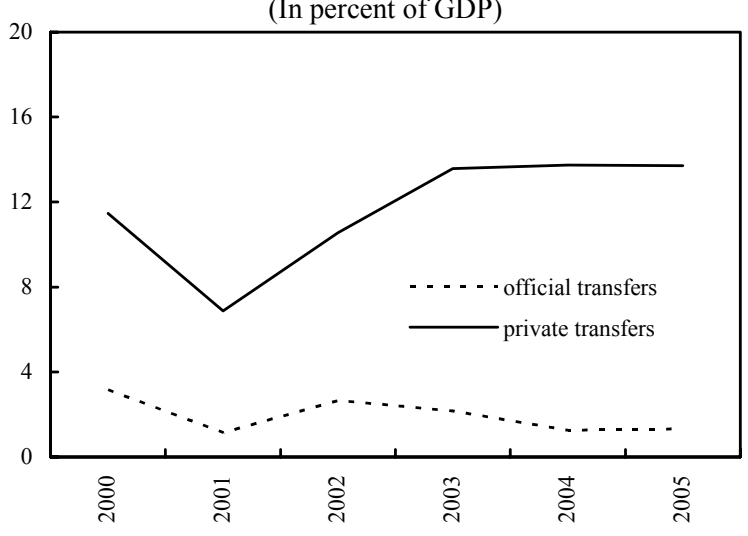

Reserve Cover

(In months of following year's imports)

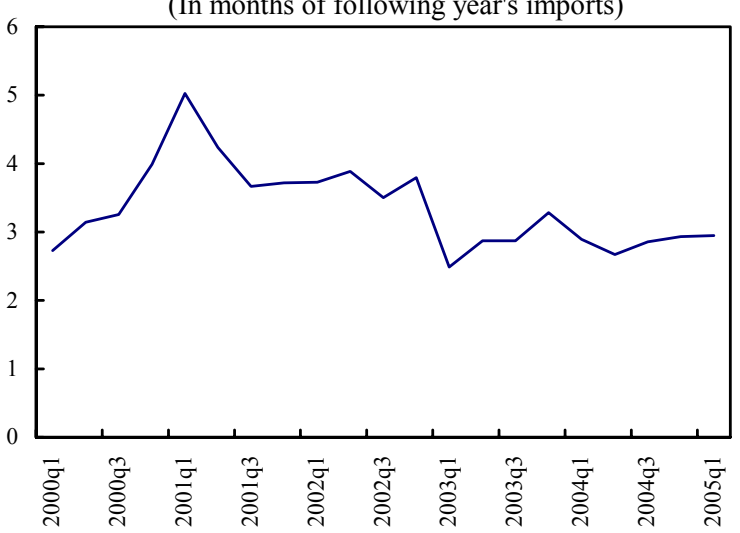

Sources: FYR Macedonian authorities; and IMF staff estimates.

$1 /$ In euros, seasonally adjusted.

2/ A sharp increase in imports took place prior to the introduction of the VAT in March 2000. 
Figure 5. FYR Macedonia: Recent Developments and Medium-Term Projections, 2000-09 1/
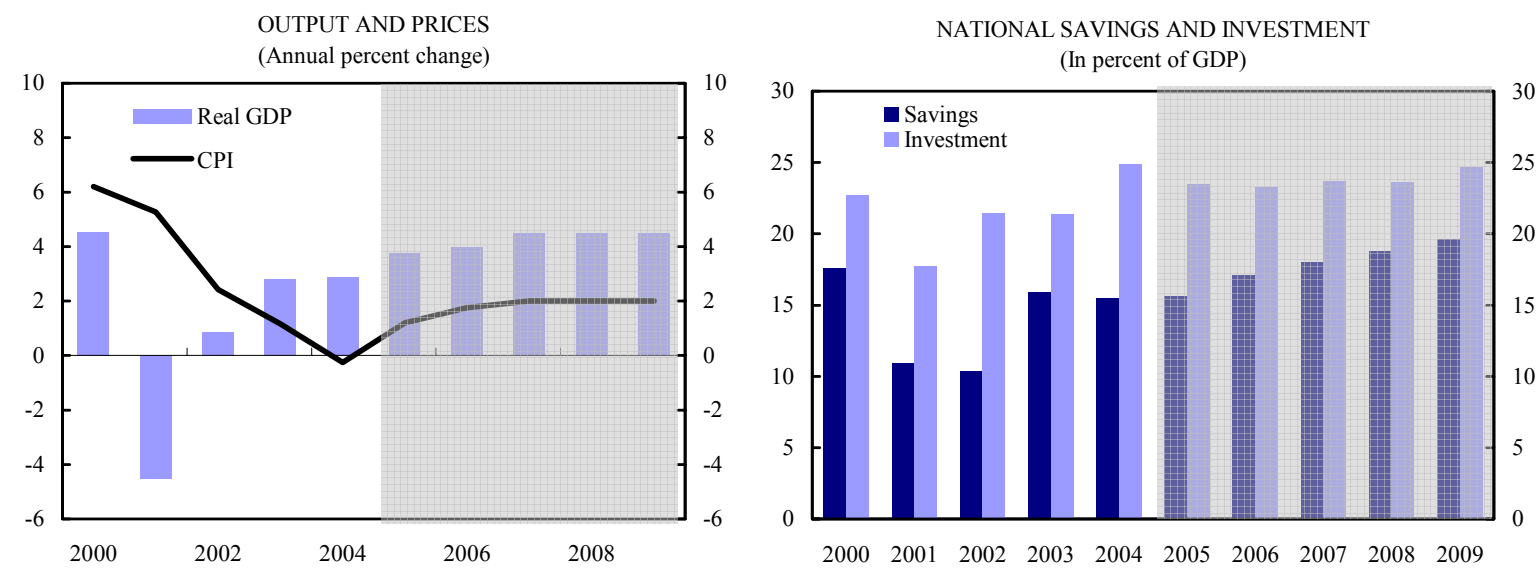

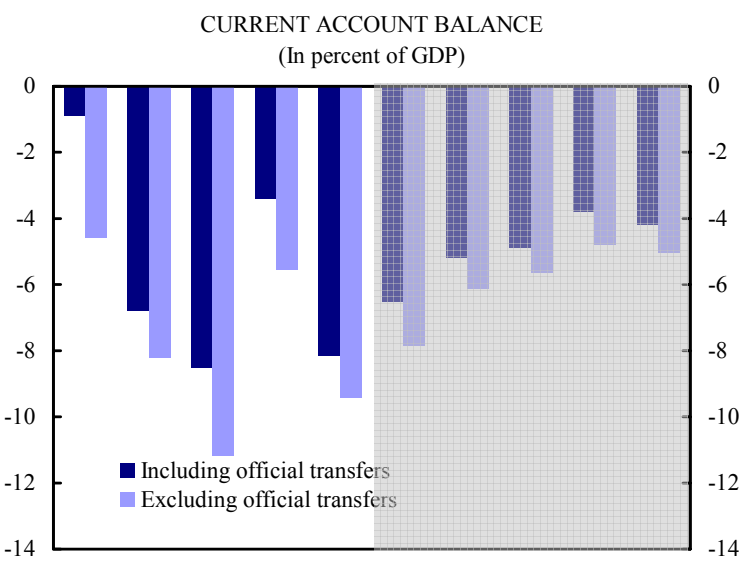

2000200120022003200420052006200720082009

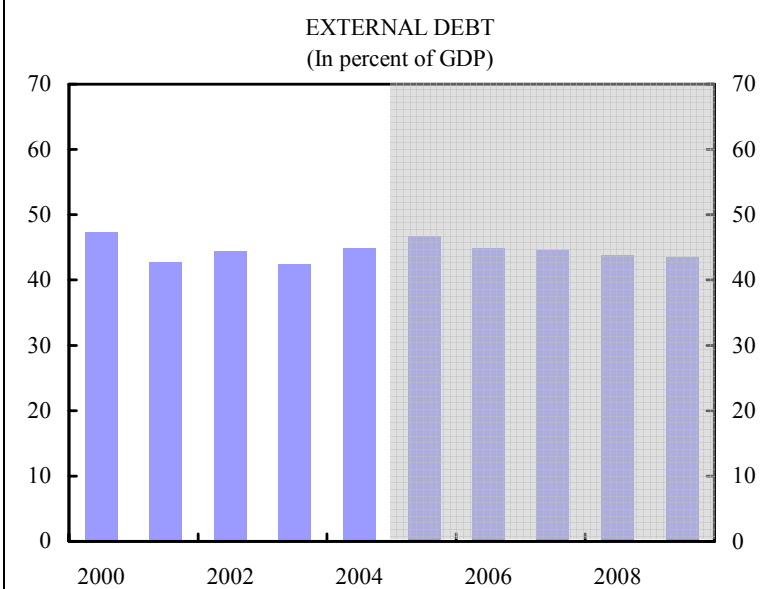

CENTRAL GOVERNMENT BALANCE

(In percent of GDP)

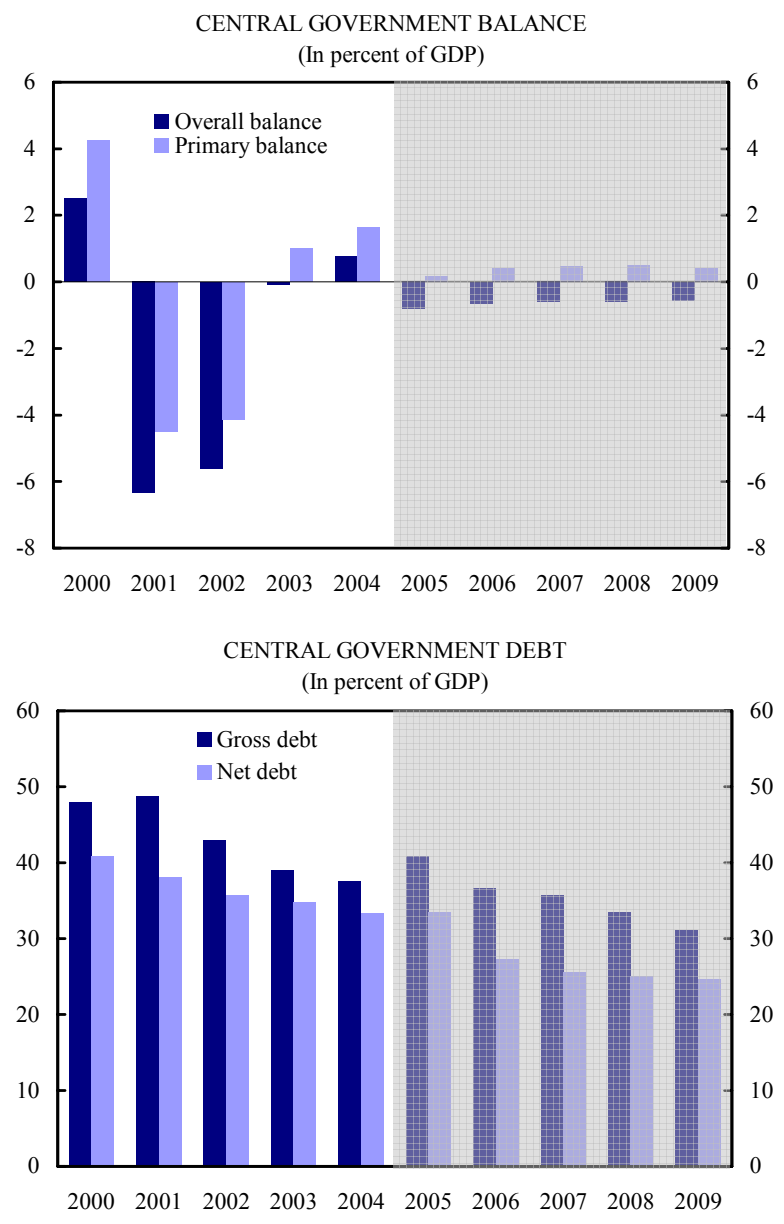

$2000 \quad 2001 \quad 2002 \quad 2003 \quad 2004 \quad 2005 \quad 2006 \quad 2007 \quad 2008 \quad 2009$

CENTRAL GOVERNMENT DEBT

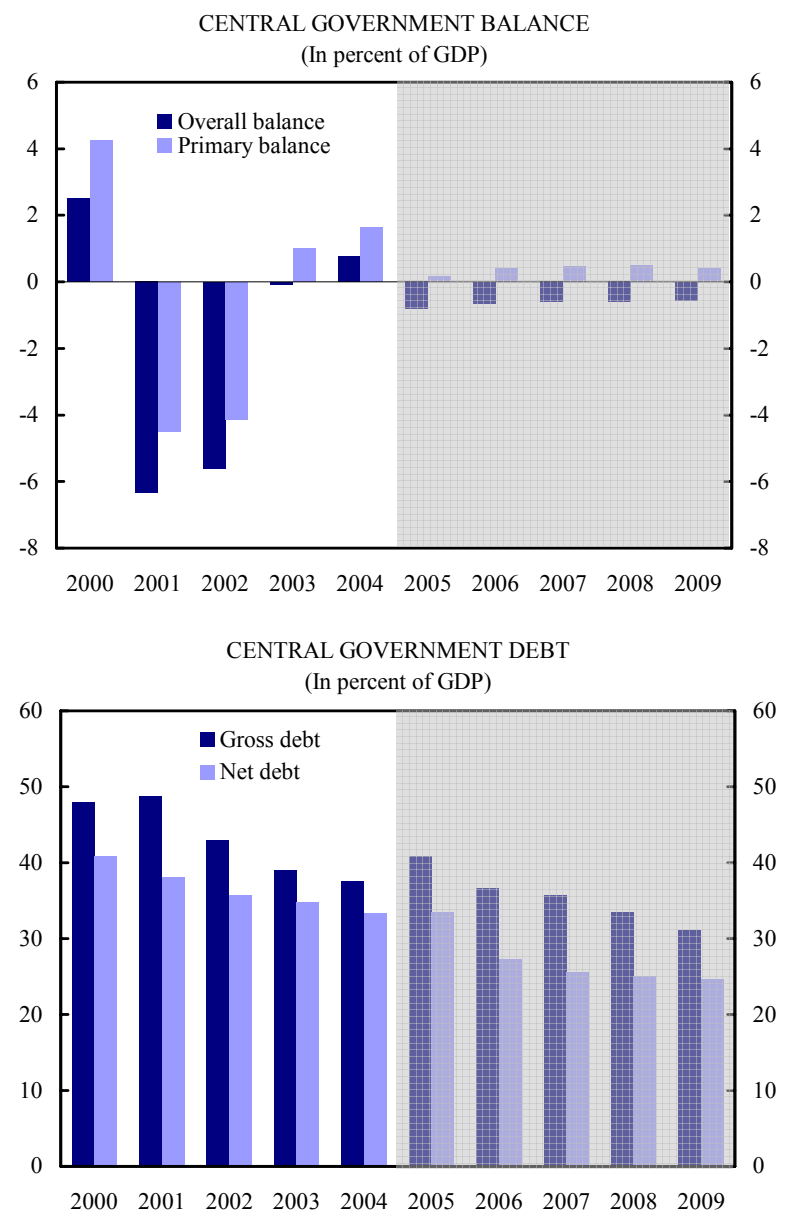

Sources: Macedonian authorities; and Fund staff estimates.

1/ 2005 onward: Projections. 
Table 1. FYR Macedonia: Selected Economic Indicators, 2000-08

\begin{tabular}{|c|c|c|c|c|c|c|c|c|c|}
\hline & 2000 & 2001 & 2002 & 2003 & $\begin{array}{l}2004 \\
\text { Prel. }\end{array}$ & 2005 & 2006 & $\frac{2007}{\text { Proj }}$ & 2008 \\
\hline Real economy & \multicolumn{9}{|c|}{ (Percent change) } \\
\hline Real GDP & 4.5 & -4.5 & 0.9 & 2.8 & 2.9 & 3.7 & 4.0 & 4.5 & 4.5 \\
\hline $\begin{array}{l}\text { Consumer prices } 1 / \\
\text { period average }\end{array}$ & & & & & & & & & \\
\hline $\begin{array}{l}\text { period average } \\
\text { end of period }\end{array}$ & 5.8 & 5.3 & 2.4 & 1.2 & -0.3 & 1.2 & 1.8 & 2.0 & 2.0 \\
\hline end of period & 6.1 & 3.7 & 1.0 & 2.6 & -2.0 & $\ldots$ & $\ldots$ & $\ldots$ & $\ldots$ \\
\hline Real wages, period average & -0.3 & -2.0 & 5.1 & 3.6 & 4.4 & $\ldots$ & $\ldots$ & $\ldots$ & $\ldots$ \\
\hline Unemployment rate (average) & 32.2 & 30.5 & 31.9 & 36.7 & 35.6 & $\ldots$ & $\ldots$ & $\ldots$ & $\ldots$ \\
\hline Government finances & \multicolumn{9}{|c|}{ (In percent of nominal GDP) } \\
\hline Central government balance 2/ 3/ & 2.5 & -6.3 & -5.6 & -0.1 & 0.7 & -0.8 & -0.6 & -0.6 & -0.6 \\
\hline Revenues (including grants) & 36.2 & 34.0 & 34.9 & 38.4 & 37.5 & 38.9 & 35.4 & 34.1 & 33.2 \\
\hline Expenditures & 33.7 & 40.3 & 40.5 & 38.5 & 36.8 & 39.7 & 36.1 & 34.7 & 33.8 \\
\hline Public sector balance & 1.8 & -7.2 & -5.6 & -0.7 & 0.3 & -1.5 & -1.3 & -1.1 & 0.4 \\
\hline \multicolumn{10}{|l|}{ Central Government debt } \\
\hline Gross & 48.0 & 48.8 & 42.9 & 39.0 & 37.6 & 40.9 & 36.6 & 35.8 & 33.4 \\
\hline Net $4 /$ & 40.8 & 38.0 & 35.8 & 34.9 & 33.3 & 33.5 & 27.3 & 25.6 & 25.0 \\
\hline Money and credit & \multicolumn{9}{|c|}{ (Percent change, end of period) } \\
\hline Broad money (M3) 5/ & 21.2 & 62.6 & -10.0 & 18.0 & 16.1 & 17.7 & 18.4 & $\ldots$ & $\ldots$ \\
\hline Total credit to private sector $6 /$ & 18.7 & 2.1 & -0.9 & 19.0 & 25.0 & 22.7 & 19.1 & $\ldots$ & $\ldots$ \\
\hline Short-term lending rate (percent) & 19.0 & 19.2 & 17.7 & 14.5 & 11.8 & $\ldots$ & $\ldots$ & $\ldots$ & $\ldots$ \\
\hline Interbank money market rate (percent) & 7.2 & 11.9 & 14.4 & 6.8 & 8.3 & $\ldots$ & $\ldots$ & $\ldots$ & $\ldots$ \\
\hline Balance of payments & \multicolumn{9}{|c|}{ (In millions of Euro) } \\
\hline Exports & 1,220 & 1,035 & 1,178 & 1,207 & 1,344 & 1,640 & 1,755 & 1,893 & 2,043 \\
\hline Imports & 1,858 & 1,502 & 1,989 & 1,961 & 2,244 & 2,517 & 2,636 & 2,792 & 2,958 \\
\hline Trade balance & -638 & -467 & -811 & -754 & -900 & -877 & -881 & -899 & -915 \\
\hline \multicolumn{10}{|l|}{ Current account balance } \\
\hline excluding grants & -198 & -262 & -443 & -231 & -396 & -345 & -287 & -281 & -255 \\
\hline (in percent of GDP) & -5.1 & -6.8 & -11.1 & -5.6 & -9.4 & -7.8 & -6.1 & -5.6 & -4.8 \\
\hline including grants & -76 & -218 & -337 & -142 & -343 & -288 & -242 & -244 & -202 \\
\hline (in percent of GDP) & -2.0 & -5.7 & -8.4 & -3.5 & -8.2 & -6.5 & -5.2 & -4.9 & -3.8 \\
\hline Overall balance & 209 & 106 & -138 & 20 & -25 & 212 & 169 & 125 & 101 \\
\hline $\begin{array}{l}\text { Official gross reserves } \\
\text { (in months of following year's imports }\end{array}$ & 752 & 857 & 692 & 715 & 717 & 916 & 1,078 & 1,190 & 1,277 \\
\hline of goods and services) & 5.2 & 4.5 & 3.8 & 3.3 & 2.9 & 3.6 & 4.0 & 4.1 & 4.1 \\
\hline External debt service ratio $7 /$ & 14.9 & 18.5 & 22.0 & 19.3 & 13.9 & 15.6 & 24.7 & 17.5 & 16.9 \\
\hline External debt to GDP ratio (percent) 8/ & 47.3 & 42.7 & 44.4 & 42.4 & 44.8 & 46.7 & 44.9 & 44.6 & 43.9 \\
\hline Exchange rates & \multicolumn{9}{|c|}{ (Percent change, period average) } \\
\hline Real effective exchange rate (CPI-based) & -3.9 & -4.3 & -1.7 & 2.7 & -1.8 & $\ldots$ & $\ldots$ & $\ldots$ & $\ldots$ \\
\hline Real effective exchange rate (ULC-based) & 10.8 & -6.3 & 4.6 & -1.1 & 1.1 & $\ldots$ & $\ldots$ & $\ldots$ & $\ldots$ \\
\hline
\end{tabular}

Sources: Data provided by the authorities; and IMF staff projections.

1/ Recent revisions by the State Statistics Office have not been incorporate in this document.

2/ Revised definitions are used starting in 2003.

3/ 0.4 percent of GDP of the 2005 fiscal account deficit is caused by the NBRM recapitalization.

4/ Gross debt minus government's deposits with the NBRM.

5/ The sharp increase of Broad Money in 2001 was caused by the Euro introduction.

6/ 2001-02 credit growth affected by loan loss provisions.

$7 /$ Debt service due, including IMF, as a percentage of exports of goods and services. Includes for 2006 a major debt restructuring operation. Excludes the roll-over of trade credits.

8/ Total external debt, including trade credit. Includes for 2005 an $€ 150$ million Eurobond issuance. 
Table 2. FYR Macedonia: Central Government Operations, 2000-08 1/

\begin{tabular}{|c|c|c|c|c|c|c|c|c|c|}
\hline & 2000 & 2001 & 2002 & 2003 & 2004 & $\begin{array}{r}2005 \\
\text { Revised } \\
\text { Budget }\end{array}$ & $\begin{array}{l}2006 \\
\text { Proj. }\end{array}$ & $\begin{array}{l}2007 \\
\text { Proj. }\end{array}$ & $\begin{array}{l}2008 \\
\text { Proj. }\end{array}$ \\
\hline & \multicolumn{9}{|c|}{ (in millions of denars) } \\
\hline Total revenue and grants & 85,533 & 79,453 & 85,204 & 96,514 & 96,872 & 105,268 & 101,615 & 104,296 & 108,098 \\
\hline Tax revenue & 77,009 & 73,644 & 80,569 & 77,056 & 81,579 & 83,847 & 85,918 & 88,492 & 92,292 \\
\hline Taxes on income and profits & 13,586 & 10,253 & 10,140 & 10,773 & 10,068 & 10,802 & 11,087 & 11,428 & 11,831 \\
\hline Domestic taxes on goods \& services & 30,537 & 28,529 & 31,923 & 32,415 & 36,753 & 37,782 & 39,605 & 41,538 & 43,534 \\
\hline VAT & 17,452 & 17,133 & 20,521 & 21,175 & 25,757 & 26,325 & 27,865 & 29,268 & 30,660 \\
\hline Excises & 13,085 & 11,397 & 11,402 & 11,240 & 10,996 & 11,457 & 11,740 & 12,270 & 12,874 \\
\hline Import duties & 7,733 & 6,111 & 6,335 & 6,140 & 5,815 & 4,867 & 4,395 & 4,033 & 3,803 \\
\hline Other taxes & 63 & 3,387 & 6,677 & 511 & 843 & 823 & 756 & 786 & 786 \\
\hline Non-tax and capital revenue & 5,284 & 5,307 & 4,600 & 16,079 & 13,577 & 17,185 & 13,197 & 13,304 & 13,306 \\
\hline Non-tax revenue & 4,807 & 4,802 & 4,005 & 15,494 & 12,956 & 13,275 & 12,372 & 12,502 & 12,656 \\
\hline Capital revenue 3 / & 477 & 505 & 595 & 584 & 621 & 3,911 & 825 & 802 & 650 \\
\hline Total expenditures & 79,628 & 94,299 & $\mathbf{9 8 , 7 5 8}$ & 96,708 & 94,944 & 107,503 & 103,466 & 106,173 & 110,055 \\
\hline Total expenditures, w/o errors \& omissions & 79,412 & 93,988 & 98,351 & 95,858 & 95,049 & 107,504 & 103,466 & 106,176 & 110,055 \\
\hline Current expenditure & 73,085 & 85,863 & 89,513 & 85,549 & 86,716 & 92,759 & 90,846 & 93,295 & $\mathbf{9 7 , 5 1 2}$ \\
\hline Goods and services & 24,207 & 37,907 & $\mathbf{3 4 , 5 5 7}$ & 33,998 & 34,232 & 37,442 & 35,301 & 28,775 & 29,219 \\
\hline Wages and salaries 4/ & 16,863 & 16,946 & 19,012 & 21,485 & 22,032 & 23,179 & 23,767 & 16,807 & 16,813 \\
\hline Goods and nonlabor services & 7,191 & 20,795 & 15,358 & 12,307 & 12,063 & 14,074 & 11,384 & 11,778 & 12,216 \\
\hline Reserves & 154 & 166 & 187 & 206 & 138 & 190 & 150 & 190 & 190 \\
\hline Transfers & 44,717 & 43,597 & 51,377 & 48,811 & 50,163 & 52,658 & 52,506 & 60,852 & 62,553 \\
\hline Interest & 4,161 & 4,359 & 3,579 & 2,740 & 2,320 & 2,659 & 3,039 & 3,270 & 3,544 \\
\hline Capital expenditure & 6,327 & 8,125 & 8,838 & 10,310 & 8,356 & 14,745 & 12,620 & 12,881 & 12,543 \\
\hline Lending minus repayments & $\ldots$ & $\ldots$ & $\ldots$ & 0 & -23 & -1 & 0 & 0 & 0 \\
\hline Statistical discrepancy/Measures & 215 & 311 & 408 & 850 & -105 & 0 & 0 & -3 & 0 \\
\hline Consolidated Central Govt Fiscal Balance & 5,905 & $-14,846$ & $-13,554$ & -194 & 1,928 & $-2,235$ & $-1,851$ & $-1,877$ & $-1,957$ \\
\hline Balance excluding NBRM recapitalization & & & & & & $-1,015$ & & & \\
\hline Central bank & $-9,367$ & $-5,320$ & 7,780 & 2,080 & $-1,025$ & $-9,085$ & $-6,692$ & $-4,358$ & 3,712 \\
\hline Commercial banking system & $-1,079$ & -226 & 593 & -454 & 364 & 845 & -26 & 224 & 474 \\
\hline $\mathrm{o} / \mathrm{w}$ T-bills issue (starting 2004) & $\ldots$ & $\ldots$ & $\ldots$ & $\ldots$ & 611 & 1,001 & 500 & 750 & 1,000 \\
\hline Other domestic financing & $-1,547$ & 0 & 1,872 & $-3,688$ & $-2,195$ & $-2,189$ & $-1,541$ & $-1,665$ & $-1,783$ \\
\hline Privatization receipts & 2,637 & 24,709 & 1,582 & 848 & 510 & 511 & 15,752 & 6,725 & 120 \\
\hline Foreign & 3,450 & $-4,317$ & 1,727 & 1,408 & 418 & 12,153 & $-5,641$ & 951 & -566 \\
\hline Unidentified financing & 0 & $\mathbf{0}$ & 0 & 0 & $\mathbf{0}$ & o & 0 & 0 & 0 \\
\hline \multicolumn{10}{|l|}{ Memorandum item: } \\
\hline Nominal GDP & 236,389 & 233,841 & 243,971 & 251,486 & 258,078 & 270,959 & 286,737 & 305,632 & 325,774 \\
\hline
\end{tabular}

\footnotetext{
Sources: Data provided by the authorities; and IMF staff projections.
}

1/ Revised definitions, incorporating special revenue accounts, foreign financial projects and grants, are used starting from 2003. (Prior to 2004, bank financing flows differ from the ones that can be derived from monetary statistics).

2/ The part of pension contributions that is expected to flow into the second pillar pension starting in October 2005 is not included.

3/ From 2005 onwards capital revenue includes dividends, interest and other property income previously reported under non-tax revenue.

4/ At the second phase of decentralization, expected to start in 2007 , wages paid by the central government decline, which is offset by higher transfers to local governments. 
Table 2. FYR Macedonia: Central Government Operations, 2000-08 1/ (continued)

\begin{tabular}{|c|c|c|c|c|c|c|c|c|c|}
\hline & 2000 & 2001 & 2002 & 2003 & 2004 & $\begin{array}{r}2005 \\
\text { Revised } \\
\text { Budget } \\
\end{array}$ & $\begin{array}{l}2006 \\
\text { Proj. }\end{array}$ & $\begin{array}{l}2007 \\
\text { Proj. }\end{array}$ & $\begin{array}{l}2008 \\
\text { Proj. }\end{array}$ \\
\hline & \multicolumn{9}{|c|}{ (in percent of GDP) } \\
\hline Total revenue and grants & 36.2 & 34.0 & 34.9 & 38.4 & 37.5 & 38.9 & 35.4 & 34.1 & 33.2 \\
\hline Tax revenue & 32.6 & 31.5 & 33.0 & 30.6 & 31.6 & 30.9 & 30.0 & 29.0 & 28.3 \\
\hline Taxes on income and profits & 5.7 & 4.4 & 4.2 & 4.3 & 3.9 & 4.0 & 3.9 & 3.7 & 3.6 \\
\hline Social insurance contributions $2 /$ & 10.6 & 10.8 & 10.4 & 10.8 & 10.9 & 10.9 & 10.5 & 10.0 & 9.9 \\
\hline Domestic taxes on goods \& services & 12.9 & 12.2 & 13.1 & 12.9 & 14.2 & 13.9 & 13.8 & 13.6 & 13.4 \\
\hline VAT & 7.4 & 7.3 & 8.4 & 8.4 & 10.0 & 9.7 & 9.7 & 9.6 & 9.4 \\
\hline Excises & 5.5 & 4.9 & 4.7 & 4.5 & 4.3 & 4.2 & 4.1 & 4.0 & 4.0 \\
\hline Import duties & 3.3 & 2.6 & 2.6 & 2.4 & 2.3 & 1.8 & 1.5 & 1.3 & 1.2 \\
\hline Other taxes & 0.0 & 1.4 & 2.7 & 0.2 & 0.3 & 0.3 & 0.3 & 0.3 & 0.2 \\
\hline Non-tax and capital revenue & 2.2 & 2.3 & 1.9 & 6.4 & 5.3 & 6.3 & 4.6 & 4.4 & 4.1 \\
\hline Non-tax revenue & 2.0 & 2.1 & 1.6 & 6.2 & 5.0 & 4.9 & 4.3 & 4.1 & 3.9 \\
\hline Capital revenue 3 / & 0.2 & 0.2 & 0.2 & 0.2 & 0.2 & 1.4 & 0.3 & 0.3 & 0.2 \\
\hline Grants & 1.4 & 0.2 & 0.0 & 1.3 & 0.7 & 1.6 & 0.9 & 0.8 & 0.8 \\
\hline Total expenditures & 33.7 & 40.3 & 40.5 & 38.5 & 36.8 & 39.7 & 36.1 & 34.7 & 33.8 \\
\hline Total expenditures, w/o errors $\&$ omissions & 33.6 & 40.2 & 40.3 & 38.1 & 36.8 & 39.7 & 36.1 & 34.7 & 33.8 \\
\hline Current expenditure & 30.9 & 36.7 & 36.7 & 34.0 & 33.6 & 34.2 & 31.7 & 30.5 & 29.9 \\
\hline Goods and services & 10.2 & 16.2 & 14.2 & 13.5 & 13.3 & 13.8 & 12.3 & 9.4 & 9.0 \\
\hline Wages and salaries $4 /$ & 7.1 & 7.2 & 7.8 & 8.5 & 8.5 & 8.6 & 8.3 & 5.5 & 5.2 \\
\hline Goods and nonlabor services & 3.0 & 8.9 & 6.3 & 4.9 & 4.7 & 5.2 & 4.0 & 3.9 & 3.7 \\
\hline Reserves & 0.1 & 0.1 & 0.1 & 0.1 & 0.1 & 0.1 & 0.1 & 0.1 & 0.1 \\
\hline Transfers & 18.9 & 18.6 & 21.1 & 19.4 & 19.4 & 19.4 & 18.3 & 19.9 & 19.2 \\
\hline Interest & 1.8 & 1.9 & 1.5 & 1.1 & 0.9 & 1.0 & 1.1 & 1.1 & 1.1 \\
\hline Capital expenditure & 2.7 & 3.5 & 3.6 & 4.1 & 3.2 & 5.4 & 4.4 & 4.2 & 3.9 \\
\hline Lending minus repayments & $\ldots$ & $\ldots$ & $\ldots$ & 0.0 & 0.0 & 0.0 & 0.0 & 0.0 & 0.0 \\
\hline Statistical discrepancy/Measures & 0.1 & 0.1 & 0.2 & 0.3 & 0.0 & 0.0 & 0.0 & 0.0 & 0.0 \\
\hline Consolidated Central Govt Fiscal Balance & 2.5 & -6.3 & -5.6 & -0.1 & 0.7 & -0.8 & -0.6 & -0.6 & -0.6 \\
\hline Balance excluding NBRM recapitalization & & & & & & -0.4 & & & \\
\hline Financing & -2.5 & 6.3 & 5.6 & 0.1 & -0.7 & 0.8 & 0.6 & 0.6 & 0.6 \\
\hline Domestic & -4.0 & 8.2 & 4.8 & -0.5 & -0.9 & -3.7 & 2.6 & 0.3 & 0.8 \\
\hline Bank financing & -4.4 & -2.4 & 3.4 & 0.6 & -0.3 & -3.0 & -2.3 & -1.4 & 1.3 \\
\hline Central bank & -4.0 & -2.3 & 3.2 & 0.8 & -0.4 & -3.4 & -2.3 & -1.4 & 1.1 \\
\hline Commercial banking system & -0.5 & -0.1 & 0.2 & -0.2 & 0.1 & 0.3 & 0.0 & 0.1 & 0.1 \\
\hline o/w T-bills issue (starting 2004) & $\ldots$ & $\ldots$ & $\ldots$ & $\ldots$ & 0.2 & 0.4 & 0.2 & 0.2 & 0.3 \\
\hline Other domestic financing & -0.7 & 0.0 & 0.8 & -1.5 & -0.9 & -0.8 & -0.5 & -0.5 & -0.5 \\
\hline Privatization receipts & 1.1 & 10.6 & 0.6 & 0.3 & 0.2 & 0.2 & 5.5 & 2.2 & 0.0 \\
\hline Foreign & 1.5 & -1.8 & 0.7 & 0.6 & 0.2 & 4.5 & -2.0 & 0.3 & -0.2 \\
\hline Unidentified financing & 0.0 & $\mathbf{0 . 0}$ & 0.0 & 0.0 & 0.0 & 0.0 & $\mathbf{0 . 0}$ & 0.0 & $\mathbf{0 . 0}$ \\
\hline
\end{tabular}

Sources: Data provided by the authorities; and IMF staff projections.

1/ Revised definitions, incorporating special revenue accounts, foreign financial projects and grants, are used starting from 2003. (Prior to 2004, bank financing flows differ from the ones that can be derived from monetary statistics).

2/ The part of pension contributions that is expected to flow into the second pillar pension strting in October 2005 is not included.

3/ From 2005 onwards capital revenue includes dividends, interest and other property income previously reported under non-tax revenue.

4/ At the second phase of decentralization, expected to start in 2007, wages paid by the central government decline, wich is offset by higher transfers to local governments. 
Table 3. FYR Macedonia: The Central Bank Accounts, 2002-06 (Millions MKD, current exchange rate)

\begin{tabular}{|c|c|c|c|c|c|c|c|c|c|c|c|}
\hline & \multirow{2}{*}{$\begin{array}{r}2002 \\
\begin{array}{r}\text { Dec. } \\
\text { Actual }\end{array}\end{array}$} & \multirow{2}{*}{$\begin{array}{r}2003 \\
\text { Dec. } \\
\text { Actual }\end{array}$} & \multirow{2}{*}{$\begin{array}{r}2004 \\
\text { Dec. } \\
\text { Actual }\end{array}$} & \multicolumn{4}{|c|}{2005} & \multicolumn{4}{|c|}{2006} \\
\hline & & & & $\begin{array}{r}\text { Mar. } \\
\text { Actual }\end{array}$ & $\begin{array}{l}\text { Jun. } \\
\text { Proj. }\end{array}$ & $\begin{array}{l}\text { Sep. } \\
\text { Proj. }\end{array}$ & $\begin{array}{l}\text { Dec. } \\
\text { Proj. }\end{array}$ & $\begin{array}{l}\text { Mar. } \\
\text { Proj. }\end{array}$ & $\begin{array}{l}\text { Jun. } \\
\text { Proj. }\end{array}$ & $\begin{array}{l}\text { Sep. } \\
\text { Proj. }\end{array}$ & $\begin{array}{l}\text { Dec. } \\
\text { Proj. }\end{array}$ \\
\hline Net foreign assets & 41,852 & 42,501 & 41,824 & 43,139 & 46,216 & 45,355 & 54,434 & 61,829 & 62,324 & 64,414 & 64,806 \\
\hline Net domestic assets & $-23,677$ & $-21,479$ & $-20,713$ & $-21,358$ & $-23,593$ & $-22,636$ & $-30,491$ & $-38,557$ & $-38,341$ & $-40,282$ & $-39,270$ \\
\hline Banks (net) & $-2,784$ & $-4,297$ & $-4,498$ & $-5,983$ & $-7,383$ & $-4,845$ & $-3,239$ & $-2,144$ & $-3,981$ & $-6,856$ & $-5,076$ \\
\hline Credits & 214 & 82 & 54 & 51 & 51 & 51 & 51 & 51 & 51 & 51 & 51 \\
\hline Instruments (NBRM bills) & $-2,998$ & $-4,379$ & $-4,552$ & $-6,034$ & $-7,434$ & $-4,896$ & $-3,290$ & $-2,195$ & $-4,032$ & $-6,907$ & $-5,127$ \\
\hline Government (net) $1 /$ & $-9,857$ & $-7,050$ & $-8,075$ & $-6,751$ & $-7,461$ & $-8,542$ & $-17,158$ & $-26,319$ & $-24,141$ & $-23,207$ & $-23,850$ \\
\hline Other items (net) & $-11,036$ & $-10,132$ & $-8,140$ & $-8,624$ & $-8,749$ & $-9,249$ & $-10,094$ & $-10,094$ & $-10,219$ & $-10,219$ & $-10,344$ \\
\hline Reserve money & 18,175 & 21,022 & 21,111 & 21,781 & 22,624 & 22,718 & 23,943 & 23,273 & 23,983 & 24,132 & 25,536 \\
\hline Currency & 14,136 & 14,177 & 14,162 & 13,207 & 13,578 & 13,362 & 14,162 & 13,320 & 13,505 & 13,280 & 14,120 \\
\hline Other & 4,039 & 6,845 & 6,949 & 8,574 & 9,046 & 9,356 & 9,781 & 9,953 & 10,478 & 10,852 & 11,416 \\
\hline Cash in vaults & 679 & 833 & 909 & 796 & 796 & 796 & 796 & 796 & 796 & 796 & 796 \\
\hline Total reserves & 3,360 & 6,012 & 6,040 & 7,778 & 8,250 & 8,560 & 8,985 & 9,157 & 9,682 & 10,056 & 10,620 \\
\hline on denar deposits & 3,360 & 3,242 & 2,674 & 3,051 & 3,579 & 3,721 & 3,945 & 3,957 & 4,237 & 4,437 & 4,711 \\
\hline \multicolumn{12}{|c|}{ Memo items: } \\
\hline \multicolumn{12}{|c|}{ Central bank accounts in constant exchange rate } \\
\hline Net foreign assets & & & 41,824 & 42,553 & 45,630 & 44,769 & 53,848 & 61,243 & 61,738 & 63,828 & 64,220 \\
\hline Net domestic assets & & & $-20,713$ & $-20,781$ & $-23,016$ & $-22,059$ & $-29,914$ & $-37,980$ & $-37,764$ & $-39,705$ & $-38,693$ \\
\hline $\mathrm{o} / \mathrm{w}$ credit to the government & & & $-8,075$ & $-6,733$ & $-7,443$ & $-8,524$ & $-17,140$ & $-26,301$ & $-24,123$ & $-23,189$ & $-23,832$ \\
\hline Reserve money & & & 21,111 & 21,772 & 22,615 & 22,709 & 23,934 & 23,264 & 23,974 & 24,123 & 25,527 \\
\hline
\end{tabular}

Sources: The National Bank of Macedonia; and IMF staff projections.

1/ Financing data in the central bank balance sheet and the fiscal table differ for 2003 when the unclaimed portion of the foreign frozen currency bond was moved from the government account to a special sub account recorded under OIN.

2 / Reserve requirements on foreign exchange deposits were introduced in the second quarter of 2003. 
Table 4. FYR Macedonia: Monetary Survey, 2002-06 (Millions MKD, current exchange rate)

\begin{tabular}{|c|c|c|c|c|c|c|c|c|c|c|c|}
\hline & \multirow{2}{*}{$\begin{array}{r}2002 \\
\begin{array}{r}\text { Dec. } \\
\text { Actual }\end{array} \\
\end{array}$} & \multirow{2}{*}{$\begin{array}{r}2003 \\
\text { Dec. } \\
\text { Actual }\end{array}$} & \multirow{2}{*}{$\begin{array}{r}2004 \\
\text { Dec. } \\
\text { Actual }\end{array}$} & \multicolumn{4}{|c|}{2005} & \multicolumn{4}{|c|}{2006} \\
\hline & & & & $\begin{array}{r}\text { Mar. } \\
\text { Actual }\end{array}$ & $\begin{array}{l}\text { Jun. } \\
\text { Proj. }\end{array}$ & $\begin{array}{l}\text { Sep. } \\
\text { Proj. }\end{array}$ & $\begin{array}{l}\text { Dec. } \\
\text { Proj. }\end{array}$ & $\begin{array}{l}\text { Mar. } \\
\text { Proj. }\end{array}$ & $\begin{array}{l}\text { Jun. } \\
\text { Proj. }\end{array}$ & $\begin{array}{l}\text { Sep. } \\
\text { Proj. }\end{array}$ & $\begin{array}{l}\text { Dec. } \\
\text { Proj. }\end{array}$ \\
\hline Net foreign assets & 62,902 & 65,792 & 68,865 & 68,421 & 71,193 & 72,256 & 83,095 & 91,143 & 92,841 & 95,525 & 97,226 \\
\hline National Bank of Macedonia (NBRM) & 41,852 & 42,501 & 41,824 & 43,139 & 46,216 & 45,355 & 54,434 & 61,829 & 62,324 & 64,414 & 64,806 \\
\hline Domestic money banks (DMB) & 21,050 & 23,291 & 27,041 & 25,282 & 24,977 & 26,902 & 28,661 & 29,314 & 30,517 & 31,111 & 32,421 \\
\hline Net domestic assets & 6,263 & 15,843 & 25,916 & 29,761 & 31,698 & 33,789 & 28,419 & 23,629 & 28,070 & 29,403 & 34,859 \\
\hline Credit to the government & $-3,089$ & -545 & $-1,206$ & 763 & 207 & -832 & $-9,442$ & $-18,610$ & $-16,438$ & $-15,511$ & $-16,160$ \\
\hline Banks & 6,768 & 6,505 & 6,869 & 7,514 & 7,668 & 7,710 & 7,716 & 7,709 & 7,703 & 7,696 & 7,690 \\
\hline NBRM (net) & $-9,857$ & $-7,050$ & $-8,075$ & $-6,751$ & $-7,461$ & $-8,542$ & $-17,158$ & $-26,319$ & $-24,141$ & $-23,207$ & $-23,850$ \\
\hline Credit to the private sector & 38,396 & 45,677 & 57,097 & 60,320 & 62,340 & 65,970 & 70,055 & 74,433 & 76,827 & 77,233 & 83,464 \\
\hline Other items (net) & $-29,044$ & $-29,289$ & $-29,975$ & $-31,322$ & $-30,849$ & $-31,349$ & $-32,194$ & $-32,194$ & $-32,319$ & $-32,319$ & $-32,444$ \\
\hline Broad money (M3) & 69,165 & 81,635 & 94,781 & 98,182 & 102,891 & 106,045 & 111,514 & 114,772 & 120,910 & 124,928 & 132,086 \\
\hline Currency in circulation & 14,136 & 14,177 & 14,162 & 13,207 & 13,578 & 13,362 & 14,162 & 13,320 & 13,505 & 13,280 & 14,120 \\
\hline Municipal and public enterprise accounts & 729 & 719 & 834 & 759 & 754 & 754 & 754 & 754 & 754 & 754 & 754 \\
\hline Private denar deposits & 26,426 & 32,264 & 37,276 & 40,188 & 41,853 & 43,540 & 46,199 & 48,699 & 52,199 & 54,699 & 58,124 \\
\hline Private foreign currency deposits & 27,874 & 34,475 & 42,509 & 44,028 & 46,706 & 48,389 & 50,399 & 51,999 & 54,452 & 56,195 & 59,087 \\
\hline \multicolumn{12}{|l|}{ Memorandum items: } \\
\hline \multicolumn{12}{|l|}{ Monetary survey at constant exchange rate: } \\
\hline Net foreign assets & $\ldots$ & $\ldots$ & 68,865 & 67,652 & 70,424 & 71,487 & 82,326 & 90,374 & 92,072 & 94,756 & 96,457 \\
\hline Net domestic assets & $\ldots$ & $\ldots$ & 25,916 & 30,154 & 32,091 & 34,182 & 28,812 & 24,022 & 28,463 & 29,796 & 35,252 \\
\hline $\mathrm{o} / \mathrm{w}$ credit to the government & $\ldots$ & $\ldots$ & $-1,206$ & 739 & 183 & -856 & $-9,466$ & $-18,634$ & $-16,462$ & $-15,535$ & $-16,184$ \\
\hline Broad money (M3) & $\ldots$ & $\ldots$ & 94,781 & 97,806 & 102,515 & 105,669 & 111,138 & 114,396 & 120,534 & 124,552 & 131,710 \\
\hline M3 private denar & 41,291 & 46,441 & 51,438 & 53,395 & 55,431 & 56,902 & 60,361 & 62,019 & 65,704 & 67,979 & 72,244 \\
\hline Percent change from end of previous year & 4.7 & 12.5 & 10.8 & 3.8 & 7.8 & 10.6 & 17.3 & 2.7 & 8.9 & 12.6 & 19.7 \\
\hline M3 & 69,165 & 81,635 & 94,781 & 98,182 & 102,891 & 106,045 & 111,514 & 114,772 & 120,910 & 124,928 & 132,086 \\
\hline Percent change from end of previous year & -10.0 & 18.0 & 16.1 & 3.6 & 8.6 & 11.9 & 17.7 & 2.9 & 8.4 & 12.0 & 18.4 \\
\hline NFA contribution & $\ldots$ & 4.2 & 3.8 & -0.5 & 2.5 & 3.6 & 15.0 & 7.2 & 8.7 & 11.1 & 12.7 \\
\hline NDA contribution & $\ldots$ & 13.9 & 12.3 & 4.1 & 6.1 & 8.3 & 2.6 & -4.3 & -0.3 & 0.9 & 5.8 \\
\hline Velocity of M3 & 3.6 & 3.1 & 2.7 & 2.6 & 2.6 & 2.5 & 2.4 & 2.4 & 2.3 & 2.3 & 2.2 \\
\hline Total private sector credit & 38,396 & 45,677 & 57,097 & 60,320 & 62,340 & 65,970 & 70,055 & 74,433 & 76,827 & 77,233 & 83,464 \\
\hline Percent change from end of previous year & -0.9 & 19.0 & 25.0 & 5.6 & 9.2 & 15.5 & 22.7 & 6.2 & 9.7 & 10.2 & 19.1 \\
\hline NFA of DMBs in percent of private FX deposits 1 / & 75.5 & 75.6 & 71.5 & 68.2 & 63.5 & 65.6 & 66.9 & 66.4 & 66.0 & 65.4 & 64.9 \\
\hline Ratio of private FX deposits to private denar deposits & 1.05 & 1.07 & 1.14 & 1.10 & 1.12 & 1.11 & 1.09 & 1.07 & 1.04 & 1.03 & 1.02 \\
\hline Share of forex deposits in BM & 0.40 & 0.42 & 0.45 & 0.45 & 0.45 & 0.46 & 0.45 & 0.45 & 0.45 & 0.45 & 0.45 \\
\hline
\end{tabular}

Sources: The National Bank of Macedonia; and IMF staff projections.

1/ Includes required reserves (RR) on FX deposits. 
Table 5. FYR Macedonia: Medium-Term Balance of Payments, 2000-09

(In millions of Euros)

\begin{tabular}{|c|c|c|c|c|c|c|c|c|c|c|}
\hline & 2000 & 2001 & 2002 & 2003 & 2004 & 2005 & 2006 & 2007 & 2008 & 2009 \\
\hline & & & & Est. & Est. & & & Proj. & & \\
\hline Current account & -76 & -218 & -337 & -142 & -343 & -288 & -242 & -244 & -202 & -236 \\
\hline Excluding official transfers & -198 & -262 & -443 & -231 & -396 & -345 & -287 & -281 & -255 & -285 \\
\hline Trade balance (fob) & -638 & -467 & -811 & -754 & -900 & -877 & -881 & -899 & -915 & -980 \\
\hline Exports & 1,220 & 1,035 & 1,178 & 1,207 & 1,344 & 1,640 & 1,755 & 1,893 & 2,043 & 2,190 \\
\hline Imports & 1,858 & 1,502 & 1,989 & 1,961 & 2,244 & 2,517 & 2,636 & 2,792 & 2,958 & 3,170 \\
\hline Services (net) & 43 & -14 & -26 & -2 & -43 & -17 & -9 & 1 & 10 & 11 \\
\hline Income (net; including net interest) & -49 & -44 & -28 & -30 & -32 & -56 & -29 & -46 & -44 & -42 \\
\hline Transfers (net) & 567 & 307 & 528 & 644 & 631 & 662 & 677 & 701 & 747 & 775 \\
\hline Official & 122 & 44 & 106 & 89 & 53 & 58 & 45 & 37 & 53 & 48 \\
\hline Private & 445 & 264 & 421 & 556 & 578 & 604 & 633 & 663 & 694 & 727 \\
\hline Capital and financial account & 107 & 225 & 223 & 176 & 313 & 500 & 411 & 369 & 302 & 312 \\
\hline Capital account (net) & 1 & 1 & 9 & -5 & -4 & -4 & -4 & -4 & -4 & -4 \\
\hline Financial account & 106 & 224 & 215 & 181 & 316 & 504 & 415 & 373 & 306 & 316 \\
\hline Disbursements & 35 & 97 & 172 & 194 & 209 & 242 & 206 & 186 & 175 & 188 \\
\hline Amortization & 128 & 169 & 183 & 146 & 154 & 125 & 275 & 173 & 197 & 195 \\
\hline Direct and portfolio investment (net) & 163 & 396 & 82 & 83 & 128 & 288 & 404 & 280 & 244 & 232 \\
\hline of which : Direct investment & 163 & 396 & 82 & 80 & 120 & 133 & 399 & 274 & 238 & 226 \\
\hline Portfolio investment & 0 & 0 & 1 & 3 & 8 & 155 & 5 & 6 & 6 & 6 \\
\hline Currency and deposits (net) & -113 & 24 & 74 & -15 & -25 & 21 & -2 & -8 & -9 & -9 \\
\hline Individuals & -27 & 337 & -144 & 35 & 59 & 59 & 59 & 59 & 59 & 59 \\
\hline Commercial banks & -86 & -313 & 218 & -50 & -84 & -39 & -62 & -67 & -68 & -68 \\
\hline Short-term loans (net) & 14 & -13 & 0 & -12 & 20 & 0 & 0 & 0 & 0 & 0 \\
\hline Trade credits (net) & 135 & -112 & 70 & 77 & 137 & 79 & 82 & 87 & 92 & 99 \\
\hline Errors and omissions & 178 & 99 & -24 & -14 & 6 & 0 & 0 & 0 & 0 & 0 \\
\hline Overall balance & 209 & 106 & -138 & 20 & -25 & 212 & 169 & 125 & 101 & 75 \\
\hline Financing & -209 & -106 & 138 & -20 & 25 & -224 & -169 & -125 & -101 & -75 \\
\hline Net foreign assets (flows) & -242 & -119 & 138 & -26 & 8 & -208 & -169 & -125 & -101 & -75 \\
\hline Valuation effects on the stock of NFA (increase: -) & -20 & -19 & 0 & 18 & 3 & 0 & 0 & 0 & 0 & 0 \\
\hline Change in the stock of NFA (increase:-) & -262 & -138 & 138 & -8 & 11 & -208 & -169 & -125 & -101 & -75 \\
\hline Change in gross foreign reserves (increase:-) & -272 & -106 & 166 & -23 & -2 & -199 & -162 & -112 & -87 & -65 \\
\hline $\operatorname{IMF}($ net) & 20 & 8 & 14 & 10 & -6 & -8 & -7 & -14 & -13 & -10 \\
\hline BIS (net) & 13 & -13 & 0 & 0 & 0 & 0 & 0 & 0 & 0 & 0 \\
\hline Other (net) & -23 & -27 & -42 & 5 & 19 & 0 & 0 & 0 & 0 & 0 \\
\hline Change in arrears $1 /$ & 14 & 13 & 0 & 6 & 17 & -17 & 0 & 0 & 0 & 0 \\
\hline Rescheduling & 18 & 0 & 0 & 0 & 0 & 0 & 0 & 0 & 0 & 0 \\
\hline Financing gap & 0 & 0 & 0 & 0 & 0 & 12 & 0 & 0 & 0 & 0 \\
\hline \multicolumn{11}{|l|}{ Memorandum items: } \\
\hline Current account (in percent of GDP) & -2.0 & -5.7 & -8.4 & -3.5 & -8.2 & -6.5 & -5.2 & -4.9 & -3.8 & -4.2 \\
\hline Excluding official transfers & -5.1 & -6.8 & -11.1 & -5.6 & -9.4 & -7.8 & -6.1 & -5.6 & -4.8 & -5.0 \\
\hline Trade balance (in percent of GDP) & -16.4 & -12.2 & -20.3 & -18.4 & -21.4 & -19.9 & -18.9 & -18.1 & -17.2 & -17.3 \\
\hline Export growth rate (percent) & -3.9 & -15.2 & 13.9 & 2.5 & 11.3 & 22.0 & 7.0 & 7.9 & 7.9 & 7.2 \\
\hline Import growth rate (percent) & 3.3 & -19.2 & 32.4 & -1.4 & 14.4 & 12.2 & 4.7 & 5.9 & 5.9 & 7.2 \\
\hline Gross reserves & 752 & 857 & 692 & 715 & 717 & 916 & 1,078 & 1,190 & 1,277 & 1,343 \\
\hline (in months of following year's imports) & 5.2 & 4.5 & 3.8 & 3.3 & 2.9 & 3.6 & 4.0 & 4.1 & 4.1 & 4.1 \\
\hline External debt service ratio (in percent) $2 /$ & 14.9 & 18.5 & 22.0 & 19.3 & 13.9 & 15.6 & 24.7 & 17.5 & 16.9 & 15.5 \\
\hline External debt to GDP ratio (in percent) $3 /$ & 41.7 & 39.6 & 39.8 & 37.2 & 36.4 & 37.4 & 34.5 & 33.1 & 31.3 & 30.0 \\
\hline Short-term external debt to GDP ratio (in percent) 4/ & 5.5 & 3.2 & 4.6 & 5.2 & 8.4 & 9.3 & 10.5 & 11.6 & 12.7 & 13.6 \\
\hline Nominal GDP (in millions of euros) & 3,877 & 3,838 & 3,991 & 4,095 & 4,207 & 4,411 & 4,668 & 4,975 & 5,303 & 5,653 \\
\hline
\end{tabular}

Sources: Data provided by the authorities; and IMF staff estimates and projections.

1/ Private sector arrears.

2/ Debt service due including IMF as percent of exports of goods and services. Excluding rollover of trade credit.

3/ Medium and long-term debt including IMF.

4/ Including trade credit. 
Table 6. FYR Macedonia: Macroeconomic Framework, 1999-2008

\begin{tabular}{|c|c|c|c|c|c|c|c|c|c|c|}
\hline & 1999 & 2000 & 2001 & 2002 & 2003 & $\begin{array}{l}2004 \\
\text { Staff } \\
\text { stimate }\end{array}$ & \multicolumn{4}{|c|}{ Projection } \\
\hline & \multicolumn{10}{|c|}{ (In percent of nominal GDP) } \\
\hline $\begin{array}{l}\text { Foreign saving (including official grants) } 1 / \\
\text { excluding official grants }\end{array}$ & $\begin{array}{l}3.1 \\
0.9\end{array}$ & $\begin{array}{l}5.1 \\
2.0\end{array}$ & $\begin{array}{l}6.8 \\
5.7\end{array}$ & $\begin{array}{r}11.1 \\
8.4\end{array}$ & $\begin{array}{l}5.6 \\
3.5\end{array}$ & $\begin{array}{l}9.4 \\
8.2\end{array}$ & $\begin{array}{l}7.8 \\
6.5\end{array}$ & $\begin{array}{l}6.1 \\
5.2\end{array}$ & $\begin{array}{l}5.6 \\
4.9\end{array}$ & $\begin{array}{l}4.8 \\
3.8\end{array}$ \\
\hline $\begin{array}{l}\text { National saving 2/ } \\
\text { Government } \\
\text { Non-government }\end{array}$ & $\begin{array}{r}20.6 \\
1.1 \\
19.5\end{array}$ & $\begin{array}{r}17.6 \\
3.8 \\
13.8\end{array}$ & $\begin{array}{r}10.9 \\
-3.1 \\
14.0\end{array}$ & $\begin{array}{r}10.3 \\
-1.9 \\
12.3\end{array}$ & $\begin{array}{r}15.8 \\
2.7 \\
13.1\end{array}$ & $\begin{array}{r}15.5 \\
3.3 \\
12.1\end{array}$ & $\begin{array}{r}15.6 \\
3.1 \\
12.6\end{array}$ & $\begin{array}{r}17.1 \\
2.9 \\
14.2\end{array}$ & $\begin{array}{r}18.0 \\
2.8 \\
15.2\end{array}$ & $\begin{array}{r}18.8 \\
2.5 \\
16.3\end{array}$ \\
\hline $\begin{array}{l}\text { Gross domestic investment } \\
\text { Government } \\
\text { Non-government }\end{array}$ & $\begin{array}{r}23.8 \\
2.3 \\
21.5\end{array}$ & $\begin{array}{r}22.7 \\
3.4 \\
19.3\end{array}$ & $\begin{array}{r}17.7 \\
4.3 \\
13.4\end{array}$ & $\begin{array}{r}21.4 \\
3.7 \\
17.8\end{array}$ & $\begin{array}{r}21.3 \\
4.7 \\
16.6\end{array}$ & $\begin{array}{r}24.9 \\
3.7 \\
21.2\end{array}$ & $\begin{array}{r}23.4 \\
6.1 \\
17.4\end{array}$ & $\begin{array}{r}23.3 \\
5.1 \\
18.2\end{array}$ & $\begin{array}{r}23.7 \\
4.7 \\
18.9\end{array}$ & $\begin{array}{r}23.6 \\
2.9 \\
20.7\end{array}$ \\
\hline $\begin{array}{l}\text { Fixed investment } \\
\text { Change in stocks }\end{array}$ & $\begin{array}{r}16.6 \\
7.2\end{array}$ & $\begin{array}{r}16.2 \\
6.5\end{array}$ & $\begin{array}{r}14.8 \\
2.9\end{array}$ & $\begin{array}{r}16.6 \\
4.9\end{array}$ & $\begin{array}{r}16.7 \\
4.6\end{array}$ & $\begin{array}{r}18.4 \\
6.5\end{array}$ & $\begin{array}{r}18.9 \\
4.5\end{array}$ & $\begin{array}{r}19.3 \\
4.0\end{array}$ & $\begin{array}{r}19.7 \\
4.0\end{array}$ & $\begin{array}{r}20.6 \\
3.0\end{array}$ \\
\hline Non-government national saving minus investment & -2.0 & -5.5 & 0.5 & -5.5 & -3.4 & -9.1 & -4.8 & -3.9 & -3.7 & -4.4 \\
\hline \multicolumn{11}{|l|}{ Fiscal indicators } \\
\hline $\begin{array}{l}\text { Central government balance } \\
\text { Public sector balance }\end{array}$ & $\begin{array}{l}0.0 \\
0.0\end{array}$ & $\begin{array}{l}2.5 \\
1.8\end{array}$ & $\begin{array}{l}-6.3 \\
-7.2\end{array}$ & $\begin{array}{l}-5.6 \\
-5.6\end{array}$ & $\begin{array}{l}-0.1 \\
-0.7\end{array}$ & $\begin{array}{l}0.7 \\
0.3\end{array}$ & $\begin{array}{l}-0.8 \\
-1.5\end{array}$ & $\begin{array}{l}-0.6 \\
-1.3\end{array}$ & $\begin{array}{l}-0.6 \\
-1.1\end{array}$ & $\begin{array}{r}-0.6 \\
0.4\end{array}$ \\
\hline $\begin{array}{l}\text { Government debt 3/ } \\
\text { Gross } \\
\text { Net } 4 /\end{array}$ & $\begin{array}{l}\cdots \\
\cdots\end{array}$ & $\begin{array}{l}48.0 \\
40.8\end{array}$ & $\begin{array}{l}48.8 \\
38.0\end{array}$ & $\begin{array}{l}42.9 \\
35.8\end{array}$ & $\begin{array}{l}39.0 \\
34.9\end{array}$ & $\begin{array}{l}37.6 \\
33.3\end{array}$ & $\begin{array}{l}40.9 \\
33.5\end{array}$ & $\begin{array}{l}36.6 \\
27.3\end{array}$ & $\begin{array}{l}35.8 \\
25.6\end{array}$ & $\begin{array}{l}33.4 \\
25.0\end{array}$ \\
\hline \multicolumn{11}{|l|}{ Memorandum items: } \\
\hline GDP & 4.4 & 4.5 & -4.5 & 0.9 & 2.8 & 2.9 & 3.7 & 4.0 & 4.5 & 4.5 \\
\hline $\begin{array}{l}\text { Foreign saving including errors and } \\
\text { omissions (in percent of nominal GDP) }\end{array}$ & -5.0 & 0.1 & 3.8 & 8.8 & 3.7 & 8.3 & 6.5 & 5.2 & 4.9 & 3.8 \\
\hline Nominal GDP (billions of denars) & 209.0 & 236.4 & 233.8 & 244.0 & 251.5 & 258.1 & 271.0 & 286.7 & 305.6 & 325.8 \\
\hline
\end{tabular}

Sources: Data provided by the authorities; and IMF staff projections.

1/ External current account deficit, excluding official grants $(+)$.

2/ Equal to gross domestic investment minus foreign saving.

3/ Includes debt of central government. Figures include bonds issued in 2001 for the frozen foreign currency deposits, as well as liabilities assumed by the government as of end-March 2000 on account of bank and enterprise restructuring, but exclude obligations for retroactive payments to pensioners. 4/ Gross debt net of government deposits in the banking system. 
Table 7. FYR Macedonia: Schedule of Purchases Under the Stand-By Arrangement 1/

\begin{tabular}{llrl}
\hline \multirow{3}{*}{ Date } & \multicolumn{2}{c}{ Amount of Purchase } & Conditions \\
\cline { 2 - 4 } & $\begin{array}{c}\text { In millions } \\
\text { of SDRs }\end{array}$ & $\begin{array}{r}\text { In percent } \\
\text { of quota }\end{array}$ \\
\hline
\end{tabular}

\begin{tabular}{|c|c|c|c|}
\hline August 31, 2005 & 10.500 & 15.24 & $\begin{array}{l}\text { Board approval of the Stand-By } \\
\text { arrangement. }\end{array}$ \\
\hline November 15, 2005 & 3.43125 & 4.98 & $\begin{array}{l}\text { Observance of end-September } \\
2005 \text { performance criteria. }\end{array}$ \\
\hline February 15, 2006 & 3.43125 & 4.98 & $\begin{array}{l}\text { Observance of end-December } \\
2005 \text { performance criteria and } \\
\text { completion of the first review. }\end{array}$ \\
\hline May 15, 2006 & 3.43125 & 4.98 & $\begin{array}{l}\text { Observance of end-March } 2006 \\
\text { performance criteria. }\end{array}$ \\
\hline August 15, 2006 & 3.43125 & 4.98 & $\begin{array}{l}\text { Observance of end-June } 2006 \\
\text { performance criteria and } \\
\text { completion of the second review. }\end{array}$ \\
\hline November 15, 2006 & 3.43125 & 4.98 & $\begin{array}{l}\text { Observance of end-September } \\
2006 \text { performance criteria. }\end{array}$ \\
\hline February 15, 2007 & 3.43125 & 4.98 & $\begin{array}{l}\text { Observance of end-December } \\
2006 \text { performance criteria and } \\
\text { completion of the third review. }\end{array}$ \\
\hline May 15, 2007 & 3.43125 & 4.98 & $\begin{array}{l}\text { Observance of end-March } 2007 \\
\text { performance criteria. }\end{array}$ \\
\hline August 15, 2007 & 3.43125 & 4.98 & $\begin{array}{l}\text { Observance of end-June } 2007 \\
\text { performance criteria and } \\
\text { completion of the fourth review. }\end{array}$ \\
\hline November 15, 2007 & 3.43125 & 4.98 & $\begin{array}{l}\text { Observance of end-September } \\
2007 \text { performance criteria. }\end{array}$ \\
\hline February 15, 2008 & 3.43125 & 4.98 & $\begin{array}{l}\text { Observance of end-December } \\
2007 \text { performance criteria and } \\
\text { completion of the fifth review. }\end{array}$ \\
\hline May 15, 2008 & 3.43125 & 4.98 & $\begin{array}{l}\text { Observance of end-March } 2008 \\
\text { performance criteria. }\end{array}$ \\
\hline August 15, 2008 & 3.43125 & 4.98 & $\begin{array}{l}\text { Observance of end-June } 2008 \\
\text { performance criteria and } \\
\text { completion of the sixth review. }\end{array}$ \\
\hline Total & 51.675 & 75.00 & \\
\hline
\end{tabular}

1/ The schedule assumes that the SBA begins on August 31, 2005 for 36 months. 
Table 8. FYR Macedonia: External Financing Requirements and Sources, 2003-08 (In millions of EUR)

\begin{tabular}{|c|c|c|c|c|c|c|}
\hline & \multirow{2}{*}{$\begin{array}{r}\text { Act. } \\
2003\end{array}$} & \multirow{2}{*}{$\begin{array}{r}\text { Est. } \\
2004 \\
\end{array}$} & \multicolumn{4}{|c|}{ IMF Staff Projection } \\
\hline & & & 2005 & 2006 & 2007 & 2008 \\
\hline 1. Gross Financing Requirement & 390 & 557 & 679 & 731 & 579 & 552 \\
\hline External current account deficit (excluding official transfers) & 231 & 396 & 345 & 287 & 281 & 255 \\
\hline Debt amortization & 146 & 154 & 125 & 275 & 173 & 197 \\
\hline Multilateral 1/ & 33 & 28 & 0 & 0 & 0 & 0 \\
\hline Bilateral & 35 & 38 & 0 & 0 & 0 & 0 \\
\hline Commercial Creditors & 78 & 87 & 125 & 275 & 173 & 197 \\
\hline Gross reserves accumulation & 23 & 2 & 199 & 162 & 112 & 87 \\
\hline IMF/BIS repurchases and repayments (net) & -10 & 6 & 8 & 7 & 14 & 13 \\
\hline 2. Identified Financing & 390 & 557 & 679 & 731 & 579 & 552 \\
\hline Official creditors & 200 & 174 & 202 & 177 & 150 & 155 \\
\hline Official transfers and grants & 89 & 53 & 58 & 45 & 37 & 53 \\
\hline Debt financing & 194 & 209 & 242 & 206 & 186 & 175 \\
\hline Multilateral & 93 & 108 & 128 & 111 & 90 & 68 \\
\hline World Bank & 52 & 46 & 53 & 37 & 43 & 42 \\
\hline Other & 8 & 14 & 75 & 74 & 47 & 25 \\
\hline Bilateral & 18 & 14 & 16 & 21 & 22 & 34 \\
\hline Commercial creditors $2 /$ & 83 & 88 & 98 & 74 & 74 & 74 \\
\hline IMF/BIS purchases and disbursements 3 / & 0 & 0 & 12 & 0 & 0 & 0 \\
\hline Foreign direct investment (net) & 80 & 120 & 133 & 399 & 274 & 238 \\
\hline Other flows 4/ & 21 & 159 & 233 & 81 & 82 & 86 \\
\hline Accumulation of arrears 5/ & 6 & 17 & -17 & 0 & 0 & 0 \\
\hline 3. Financing Gap (1 - 2) & 0 & 0 & 0 & 0 & 0 & 0 \\
\hline
\end{tabular}

Sources: Data provided by the authorities; and IMF staff estimates and projections.

1/ Excluding the IMF.

2/ Includes the capitalization of moratorium interest according to the London Club agreement of 1997.

3/ The proposed SBA.

4/ Includes all other net financial flows and errors and omissions.

5/ Private sector and public enterprise arrears. 
Table 9. FYR Macedonia: Indicators of Capacity to Repay the Fund, 2003-10

(Under Obligation Schedule)

\begin{tabular}{|c|c|c|c|c|c|c|c|c|}
\hline & 2003 & 2004 & 2005 & 2006 & 2007 & 2008 & 2009 & 2010 \\
\hline & Est. & \multicolumn{7}{|c|}{ Projections } \\
\hline \multicolumn{9}{|l|}{ Fund repurchases and charges $1 /$} \\
\hline In millions of SDRs & 16.4 & 14.3 & 9.2 & 8.8 & 14.2 & 14.0 & 15.1 & 17.2 \\
\hline In millions of Euros & 21.3 & 17.8 & 10.7 & 10.2 & 16.5 & 16.2 & 17.5 & 19.8 \\
\hline In percent of exports of goods and services & 1.5 & 1.1 & 0.5 & 0.5 & 0.7 & 0.6 & 0.6 & 0.7 \\
\hline In percent of debt service & 7.7 & 7.7 & 4.4 & 2.5 & 5.2 & 4.9 & 5.4 & $\ldots$ \\
\hline In percent of quota & 23.8 & 20.8 & 13.4 & 12.7 & 20.6 & 20.3 & 22.0 & 24.9 \\
\hline In percent of gross official reserves & 3.0 & 2.5 & 1.2 & 0.9 & 1.4 & 1.3 & 1.3 & $\ldots$ \\
\hline \multicolumn{9}{|l|}{ Fund credit outstanding (e.o.p.) 1/ } \\
\hline In millions of SDRs & 46.0 & 40.3 & 44.0 & 54.2 & 55.8 & 54.4 & 41.4 & 25.7 \\
\hline In millions of Euros & 59.7 & 49.9 & 50.9 & 62.8 & 64.6 & 63.0 & 47.8 & 29.7 \\
\hline In percent of quota & 66.8 & 58.4 & 63.9 & 78.6 & 81.0 & 79.0 & 60.0 & 37.3 \\
\hline In percent of GDP & 1.5 & 1.2 & 1.2 & 1.3 & 1.3 & 1.2 & 0.8 & 0.5 \\
\hline In percent of gross official reserves & 8.3 & 7.0 & 5.6 & 5.9 & 5.5 & 4.9 & 3.5 & $\ldots$ \\
\hline \multicolumn{9}{|l|}{ Memorandum items: } \\
\hline Exports of goods and services (millions of Euros) & 1,438 & 1,672 & 2,039 & 2,181 & 2,356 & 2,542 & 2,725 & 2,921 \\
\hline Debt service (millions of Euros) 2/ & 278 & 232 & 242 & 411 & 314 & 327 & 321 & 305 \\
\hline Quota (millions of SDRs) & 69 & 69 & 69 & 69 & 69 & 69 & 69 & 69 \\
\hline Quota (millions of Euros) & 89 & 85 & 80 & 80 & 80 & 80 & 80 & 79 \\
\hline Gross official reserves (millions of Euros) & 715 & 717 & 916 & 1,078 & 1,190 & 1,277 & 1,352 & $\ldots$ \\
\hline GDP (millions of Euros) & 4,095 & 4,207 & 4,411 & 4,668 & 4,975 & 5,303 & 5,653 & 6,026 \\
\hline Euros per SDR 3/ & 1.298 & 1.239 & 1.158 & 1.159 & 1.158 & 1.157 & 1.155 & 1.153 \\
\hline
\end{tabular}

Sources: Data provided by the authorities; and IMF staff estimates and projections.

1/ Including the purchases under the proposed Stand-By Arrangement.

2/ Excluding rollover of trade credits.

3/ WEO assumptions of April 2005. 
Table 10. FYR Macedonia: Projected Payments to the Fund as of June 30, 2005 Under Obligated Repurchase Schedule (in millions of SDRs)

\begin{tabular}{|c|c|c|c|c|c|c|c|c|}
\hline & $20052 /$ & 2006 & 2007 & 2008 & 2009 & 2010 & 2011 & 2012 \\
\hline \multicolumn{9}{|c|}{ Obligations from existing drawings } \\
\hline \multicolumn{9}{|l|}{ Principal } \\
\hline EFF & 0.1 & 0.2 & 0.2 & 0.1 & 0.2 & 0.2 & 0.0 & 0.0 \\
\hline Stand-by & 0.0 & 1.0 & 7.0 & 9.0 & 3.0 & 0.0 & 0.0 & 0.0 \\
\hline PRGF Loans & 2.7 & 5.8 & 4.9 & 1.3 & 0.3 & 0.3 & 0.0 & 0.0 \\
\hline Systemic Transformation & 0.0 & 0.0 & 0.0 & 0.0 & 0.0 & 0.0 & 0.0 & 0.0 \\
\hline \multicolumn{9}{|l|}{ Charges and Interest $1 /$} \\
\hline SDR Assesments & 0.0 & 0.0 & 0.0 & 0.0 & 0.0 & 0.0 & 0.0 & 0.0 \\
\hline GRA Charges & 0.4 & 0.8 & 0.7 & 0.3 & 0.1 & 0.0 & 0.0 & 0.0 \\
\hline PRGF Interest & 0.0 & 0.1 & 0.0 & 0.0 & 0.0 & 0.0 & 0.0 & 0.0 \\
\hline SDR charges & 0.1 & 0.2 & 0.2 & 0.2 & 0.2 & 0.2 & 0.2 & 0.2 \\
\hline Total obligations & 3.3 & 8.0 & 12.9 & 10.9 & 3.8 & 0.7 & 0.2 & 0.2 \\
\hline (percent of quota) & 0.0 & 0.1 & 0.2 & 0.2 & 0.1 & 0.0 & 0.0 & 0.0 \\
\hline \multicolumn{9}{|c|}{ Obligations from prospective drawings } \\
\hline \multicolumn{9}{|l|}{ Principal } \\
\hline Stand-by & 0.0 & 0.0 & 0.0 & 1.3 & 9.5 & 15.1 & 13.7 & 9.4 \\
\hline \multicolumn{9}{|l|}{ Charges and Interest $1 /$} \\
\hline GRA Charges & 0.1 & 0.7 & 1.2 & 1.7 & 1.8 & 1.4 & 0.8 & 0.3 \\
\hline Service Charge & 0.1 & 0.1 & 0.1 & 0.1 & 0.0 & 0.0 & 0.0 & 0.0 \\
\hline Total obligations & 0.1 & 0.8 & 1.3 & 3.1 & 11.3 & 16.4 & 14.5 & 9.7 \\
\hline (percent of quota) & 0.0 & 0.0 & 0.0 & 0.0 & 0.2 & 0.2 & 0.2 & 0.1 \\
\hline \multicolumn{9}{|c|}{ Cumulative (existing and prospective) } \\
\hline \multicolumn{9}{|l|}{ Principal } \\
\hline EFF & 0.1 & 0.2 & 0.2 & 0.1 & 0.2 & 0.2 & 0.0 & 0.0 \\
\hline Stand-by & 0.0 & 1.0 & 7.0 & 10.3 & 12.5 & 15.1 & 13.7 & 9.4 \\
\hline PRGF Loans & 2.7 & 5.8 & 4.9 & 1.3 & 0.3 & 0.3 & 0.0 & 0.0 \\
\hline Systemic Transformation & 0.0 & 0.0 & 0.0 & 0.0 & 0.0 & 0.0 & 0.0 & 0.0 \\
\hline \multicolumn{9}{|l|}{ Charges and Interest $1 /$} \\
\hline SDR Assesments & 0.0 & 0.0 & 0.0 & 0.0 & 0.0 & 0.0 & 0.0 & 0.0 \\
\hline GRA Charges & 0.4 & 1.5 & 1.9 & 2.1 & 1.9 & 1.4 & 0.8 & 0.3 \\
\hline PRGF Interest & 0.0 & 0.1 & 0.0 & 0.0 & 0.0 & 0.0 & 0.0 & 0.0 \\
\hline SDR charges & 0.1 & 0.2 & 0.2 & 0.2 & 0.2 & 0.2 & 0.2 & 0.2 \\
\hline Service Charge & 0.1 & 0.1 & 0.1 & 0.1 & 0.0 & 0.0 & 0.0 & 0.0 \\
\hline Total obligations & 3.5 & 8.8 & 14.2 & 14.0 & 15.1 & 17.2 & 14.7 & 9.9 \\
\hline (percent of quota) & 0.1 & 0.1 & 0.2 & 0.2 & 0.2 & 0.2 & 0.2 & 0.1 \\
\hline
\end{tabular}

Prepared by Finance Department.

1/ The current basic rate of charge in the GRA is $3.57 \%$ plus 12 basis points for burden sharing. This rate of charge remains constant for future projections.

2/ July to December 
Financial indicators

Broad money (end of period; percent change from end of previous year)

Private sector credit (end of period; percent change from end of previous year) 1/

Share of non-performing loans in total bank exposures (end of period, in percent)

Foreign currency deposits (end of period, in percent of broad money)

$\begin{array}{rrrrrr}21.2 & 62.6 & -10.0 & 18.0 & 16.1 & 18.6 \\ 18.7 & 2.1 & -0.9 & 19.0 & 25.0 & 24.2 \\ 34.8 & 33.7 & 15.9 & 10.2 & 8.9 & 8.5 \\ 22.9 & 45.8 & 40.3 & 42.2 & 44.8 & 45.1 \\ & & & & & \\ \ldots & \ldots & \ldots & 37.6 & 41.2 & 44.9 \\ \ldots & 0.0 & 2.0 & 0.0 & 0.7 & 0.0 \\ \ldots & 876 & 609 & 624 & 714 & 747 \\ \ldots & 771 & 555 & 627 & 743 & 784 \\ 7.2 & 11.9 & 14.4 & 6.8 & 8.3 & 8.6\end{array}$

(end of period, in percent of total credit to private sector)

Central bank short-term foreign liabilities (in millions of Euro)

Short term foreign assets of commercial banks (in millions of Euro)

Short term foreign liabilities of commercial banks (in millions of Euro)

Money market rate (end of period; in percent)

\begin{tabular}{rrrrrr}
-3.9 & -15.2 & 13.9 & 2.5 & 11.3 & 22.0 \\
3.3 & -19.2 & 32.4 & -1.4 & 14.4 & 12.2 \\
& & & & & \\
-2.0 & -5.7 & -8.4 & -3.5 & -8.2 & -6.5 \\
-5.1 & -6.8 & -11.1 & -5.6 & -9.4 & -7.8 \\
4.9 & 10.3 & 2.1 & 2.0 & 2.9 & 3.0 \\
752 & 857 & 692 & 715 & 717 & 916 \\
5.2 & 4.5 & 3.8 & 3.3 & 2.9 & 3.6 \\
& & & & & \\
47.3 & 42.7 & 44.4 & 42.4 & 44.8 & 46.7 \\
34.5 & 34.3 & 30.1 & 27.4 & 26.9 & 30.3 \\
& & & & & \\
5.5 & 3.2 & 4.6 & 5.2 & 8.4 & 9.3 \\
28.3 & 14.2 & 24.9 & 27.4 & 45.9 & 46.4 \\
14.9 & 18.5 & 22.0 & 19.3 & 13.9 & 15.6 \\
66.0 & 68.0 & 64.7 & 54.3 & 49.4 & $\ldots$ \\
& & & & & $\ldots$ \\
-3.9 & -4.3 & -1.7 & 2.7 & -1.8 & $\ldots$ \\
10.8 & -6.3 & 4.6 & -1.1 & 1.1 & $\ldots$ \\
\hline
\end{tabular}

External Indicators

Exports (percent change, in terms of EUR)

Imports (percent change, in terms of EUR)

Current account balance (in percent of GDP)

(Including official grants)

(Excluding official grants)

foreign direct investment (in percent of GDP)

Gross official reserves (in EUR)

(In months of next year's imports of goods and services)

(In percent of private denar broad money)

Total external debt (in percent of GDP)

of which: public sector (in percent of GDP) 2

Short-term debt (by remaining maturity) $3 /$

(In percent of GDP)

(In percent of official reserves)

External debt service payments (in percent of exports of goods and services) $4 /$

Exchange rate (denar per U.S. dollar, period average)

REER (average percent change; (-) depreciation)

CPI-based

ULC-based

Source: Staff calculations and estimates based on the data provided by the NBM.

$a$ Latest available 2005 data or estimate.

1/ 2001-02 credit growth affected by loan loss provisions.

2/ Central government and public enterprises, excluding the NBRM.

3 / Including estimated stock of trade credit based on cumulative balance of payments flows since 2000

4/ Excluding trade credit. 
Mr. Rodrigo de Rato

Skopje, August 16, 2005

Managing Director

International Monetary Fund

Washington, DC 20431

U.S.A.

Dear Mr. de Rato,

The attached Memorandum of Economic and Financial Policies for 2005-08 (MEFP) describes a 3-year economic program that aims to increase growth and employment, reduce external imbalances, and secure macroeconomic stability. The associated Technical Memorandum of Understanding (TMU) describes the methods to be used in assessing program performance.

In support of the program we request a 36-month Stand-By Arrangement in an amount of SDR 51.675 million, equivalent to 75 percent of quota. The prior actions for the approval of the arrangement listed in Table 2 of the MEFP have been implemented.

We intend to make the first purchase that would become available upon the approval of the new arrangement. However, should macroeconomic conditions and balance of payments financing turn out to be in line with the program's baseline assumptions, or be more favorable, we will forgo further purchases and treat the remaining access as precautionary.

To smooth our debt service profile, we also request that the repurchase expectations falling due before end-September 2006 be extended to an obligations basis.

We believe that the policies set forth in the attached MEFP are adequate to achieve the objectives of our program but we will take any further measures that may become appropriate for this purpose. We will consult with the Fund on the adoption of such measures and in advance of any revisions to the policies contained in the MEFP, in accordance with the Fund's policies on such consultations.

Sincerely,

\section{Vlado Bučkovski \\ Prime Minister}

$/ \mathrm{s} /$

Nikola Popovski

Minister of Finance
$/ \mathrm{s} /$

$/ \mathrm{s} /$
Petar Gošev
Governor
National Bank of the Republic of Macedonia

Attachments (2) 


\section{FYR Macedonia \\ Memorandum on Economic and Financial Policies}

\section{INTRODUCTION}

1. This memorandum describes the 2005-08 economic program which we are asking the International Monetary Fund (IMF) to support under a three-year Stand-By Arrangement (SBA).

\section{A number of accomplishments during the last few years set the stage for a new}

arrangement. On the macroeconomic front, the main objective of the previous SBA-a return to a sustainable fiscal position after the security crisis in 2001-has been attained. Macroeconomic stability is now firmly entrenched; inflationary pressures remain subdued, and growth, while still below potential, has begun to pick up. There has also been progress on the political agenda. Key components of the Framework Agreement - territorial reorganization and fiscal decentralization - are moving ahead and the representation of nonmajority communities in state organs has increased. Furthermore, Macedonia has applied for candidacy to the European Union. For 2006, we expect an invitation for membership in NATO. These developments have contributed to positive reviews (BB+/B local currency, $\mathrm{BB} / \mathrm{B}$ foreign currency) by a major international credit rating agency (Standard and Poor's).

3. At the same time, relatively slow growth and a persistently wide current account deficit underline the need for structural reforms to complement our already prudent macroeconomic posture. As noted in the IMF's ex-post assessment (EPA), Macedonia's growth and employment have been weaker than in other transition economies-including countries that also experienced conflict-related turbulence. And the current account deficit is still at a level that raises concerns regarding competitiveness. Business climate surveys consistently indicate that deficiencies in the public sector, limited results in the fight against corruption, an inefficient and non-transparent judicial system, and limited policy predictability have impeded private sector development. Moreover, outside a few large privatizations, foreign direct investment has been slow to materialize. Against this background, a main objective of our program is to improve the business climate by increasing the flexibility of the labor market, raising the efficiency of the judicial system, and improving public sector governance and efficiency. These measures will be supported by continued prudent fiscal and monetary policies.

4. We anticipate that structural reforms and macroeconomic stability will enhance external competitiveness and accelerate growth. We expect that the growth rate of real GDP will rise to about 4.5 percent per year on average during 2005-08, with inflation rising from its current exceptionally low level to about 2 percent. The external current account deficit (excluding official grants), which one-time factors raised to an exceptional 9.4 percent of GDP in 2004, is expected to fall by $1 \frac{1}{2}$ percentage points in 2005 as these temporary factors unwind, and further to about 6 percent of GDP as structural reforms strengthen competitiveness. 


\section{EConomic ANd Financial Policies}

\section{A. Medium Term Issues}

\section{Our financial program (see Table 1) aims to manage the passage from} dependence on official balance of payments support to reliance on market financing while maintaining the fixed exchange rate that has anchored inflation in the past. This task is made more difficult by a number of factors. First, in view of our dependence on a narrow and volatile export base and the prospect of increased exposure to capital markets, a key program objective is to increase gross international reserves from three months to four months of import cover. Second, budget financing is made more difficult by declining balance of payments support at a time when our capacity to raise domestic finance is still not well developed. And, third, we need to amortize large volumes of foreign exchange indexed debt to residents - debt that compensates for expropriations that took place prior to independence.

6. Our financial strategy has three main elements. First, to keep the financing need manageable, we will keep the fiscal deficit at levels ( 0.8 percent of GDP in 2005 and 0.6 percent thereafter - approval of a 2006 budget in line with this ceiling is a performance criterion under the program) that are well below the threshold of debt sustainability. Second, to make it possible to increase international reserves, we will accelerate our sales of state assets, including through major privatizations. And, third, we are developing our capacity to borrow on commercial terms both domestically and abroad. The IMF arrangement that we are seeking will provide an additional cushion. However, if privatization inflows are in line with our (conservative) projections, we will not need to draw on the IMF after the initial purchase.

\section{In the fiscal area, our medium term strategy includes strengthening tax} collection, and improving public resource management. In the tax area we intend to broaden the tax base and we will harmonize the bases and unify the collection of the personal income tax and social contributions ( $\$ 36)$. In the expenditure area, we plan to keep wages and hiring under strict control in order to ensure that the share of salaries in tax revenues does not rise above the level in the 2005 supplementary budget. ${ }^{1}$ Additional hiring needs, including those arising from reforms and from our policy of ensuring equitable representation of ethnic communities, will be accommodated through natural attrition and improved allocation of existing human resources in line with the medium term strategy of human resource management defined below ( $(41)$.

8. Our privatization program is ambitious, extending to the electricity sector, telecommunications, banks and rental property among others. On electricity, the details

\footnotetext{
${ }^{1}$ Once fiscal decentralization moves to its second phase, transfers earmarked for municipal salaries will be included in this ratio.
} 
of a partial privatization of Electricity power company of Macedonia (ESM) are still being finalized with support from the EBRD, which is participating in the pre-privatization process, and the World Bank, which is providing an assessment of the regulatory implications. We intend to sell the distribution in the first quarter of 2006 (structural performance criterion) and part or all of our generating capacity in 2007, following further study of the regulatory implications of privatizing hydroelectric capacity. We will sell our residual shares in the telecommunication sector by mid-2006 (structural performance criterion). In addition, we will start tendering our equity in commercial banks by end-2005 (except Postal Bank and the Macedonian Bank for Development Promotion)(structural benchmark) and will sell the public company holding the state's rental property in 2007.

\section{In developing our capacity to borrow commercially we will focus on the} medium-term task of strengthening the infant treasury market while relying on external borrowing. The positive reviews of credit rating agencies have put Macedonia on the map for institutional investors. With the issuance of our first Eurobond we will generate resources to lengthen the maturity of our foreign debt and cushion the decline in official financing. At the same time, we will press ahead with the development of the domestic treasury market in order to limit our dependence on international capital markets. ( $\mathbf{9} 23$ ).

\section{B. Fiscal and Monetary Policies for 2005}

\section{The 2005 supplementary budget tightens our fiscal stance in order to contain a} large fiscal impulse and limit the need for central bank finance. The government has adopted a supplementary budget (prior action) with a central government deficit of 0.8 percent of GDP including transfers to recapitalize the NBRM and 0.4 percent excluding these transfers, which create no fiscal impulse. This represents an adjustment of 0.8 percent of GDP compared with the original 2005 budget. Stronger than anticipated tax and non-tax revenues account for around $2 / 3$ of the adjustment; the rest will be achieved by net expenditure savings and cuts. At the same time, some spending pressures (notably the need to bring the wages of appointed and elected officials in line with those of senior civil servants) will be accommodated through reallocations of expenditures.

\section{The NBRM will adopt a monetary stance that, combined with the tight} supplementary budget and expected inflows from abroad, will allow for a modest increase in the underlying reserve cover in 2005. Gross reserves are expected to reach 3.6 months of import cover (up from 3 months at end-2004) but most of the increase is made up of the proceeds of the Eurobond issue planned for late 2005, which will be used to restructure foreign debt in early 2006. (Excluding the debt restructuring, reserves will rise to only 3.1 months of import cover in 2005 but will reach 4 months in 2006, once the projected privatization proceeds have been received). Broad money is expected to grow in line with recent trends in velocity while the degree of euroization is expected to remain unchanged, reflecting the stabilization of currency preferences in 2004. Credit to the private sector is expected to increase by 23 percent, boosted by banks' lending some foreign currency deposits rather than investing them abroad. We project that these trends, combined with limits on credit to the government, should allow us to meet the reserve target without raising 
the interest rate. However, if necessary, the NBRM stands ready to use all available monetary instruments, including a further increase in the interest rate.

\section{BUSINESS ENVIRONMENT}

\section{An important goal of our program is to strengthen the business environment,} allowing Macedonian enterprises to compete more successfully in increasingly global markets. The main weaknesses in the business climate are a rigid labor market, the judicial sector's limited expertise in commercial law, a limited ability to attract strategic investors for state-owned enterprises, and the failure of newly privatized firms to restructure or innovate. The measures outlined in this MEFP will be complemented by a new World Bank Business Environment Reform and Institutional Strengthening Project. They will stimulate economic growth based on increased domestic and foreign investment by strengthening the public sector capacity to improve selected areas of the business environment and increase competitiveness of the business sector.

\section{A. Labor Market Reform}

\section{The high rate of measured unemployment is a reflection of rigid labor market} institutions and legislation. High hiring and firing costs, restrictive work place regulations, generous mandated benefits, and ineffective mechanisms for settling disputes, have combined to limit opportunities for investment and job creation. At the same time, workers are frequently left unprotected in a system where labor contracts are not respected or enforced. This environment has eroded confidence in labor contracts and led to wage and contribution arrears, recurrent employment violations, and a large informal economy.

\section{Therefore, as a prior action under the program we have enacted new labor} legislation which strikes a better balance between the protection of workers' rights and the needs of employers. The new Law on Labor Relations, which was enacted in July this year, is a broad based and extensive overhaul of previous legislation and the system of collective agreements. In particular:

- Under the new law, collective agreements, including at the general and branch level, will be binding only for signatories and members of signatory associations and trade unions, and will be effective for a fixed term, set at 2 years;

- $\quad$ Barriers to fixed-term, temporary, and part-time contracts have been removed;

- The rules for overtime have been clarified and liberalized (under the new law, only a weekly overtime limit of 10 hours and an annual limit of 190 hours applies);

- $\quad$ Redundancy procedures have been streamlined and the scope for legal challenge reduced by eliminating the need to establish the existence of a "technological surplus" and by abolishing the requirement to revise the organizational chart of a company; 
- Maximum severance payments have been reduced from 8 to 6 monthly salaries.

- The employers' obligation to pay a sick leave allowance has been reduced from 60 to 30 days. Additional costs arising from this measure for the HIF are covered by tightening conditions for the allowance and reducing its size which will be reflected in amendments in the Law on Health Insurance by September 2005.

In order to ensure that provisions of the new Law on Labor Relations become effective rapidly we will amend the Law on the Chamber of Commerce by end-2005 to allow employers to review their membership of the Chamber prior to the negotiation of new collective agreements (structural benchmark).

\section{B. Judicial Reform}

\section{Judicial reform will play a key role in facilitating investment and accelerating} private sector-led growth. In November 2004 the Government adopted a comprehensive strategy for legal and judicial reform and an action plan for its implementation. The goal of the strategy is to put in place an efficient justice system, based on European legal standards, that can support a market economy. While judicial reform will take several years to complete, we have begun to move forcefully: a number of key measures will be implemented within the next twelve months.

\section{An immediate objective is to reduce delays and strengthen the enforcement of} court decisions. Since delays are one of the main problems of Macedonian courts, we have recently (June 2005) adopted a new Law on Civil Procedures (or Law on Litigation) which should accelerate the resolution of cases by imposing stricter deadlines and sanctions for delays. And, to improve enforcement of court decisions, we have recently adopted a new Law on Enforcement that moves the enforcement of court decisions from the courts to independent specialized agents. We will use the 9-month transitional period before this law becomes effective to develop and adopt the necessary regulations and licensing procedures, train new enforcement agents, and educate the general public. We expect that the new enforcement system will start functioning in April 2006.

\section{Another overarching objective of judicial reform is to strengthen the} independence and professionalism of the judiciary. We plan to reform the selection, appointment and promotion of judges and prosecutors and make career development meritbased. By end-March 2006, we will amend key laws - notably the Law on Courts and the Law on the Judicial Council - to make the new system of judicial appointments operational. These legislative changes require Constitutional amendments, which will be completed by end-2005.

\section{We will also establish a training institution for judges and prosecutors.}

Completion of a training course in the school will be a requirement for all new judicial appointments. We will adopt the law on the establishment of the school by end-November 
2005 and will make the school operational in January 2006. The curriculum will include business economics and commercial law.

19. The court reforms will also allow more efficient handling of administrative and misdemeanor cases (structural benchmark). By end-July 2006 we will amend the Law on Misdemeanors to allow public institutions, such as the NBRM, the PRO, customs administration and others to impose sanctions for misdemeanors without prior court approval.

20. Finally, as the reform moves ahead, we will improve the allocation of human and financial resources to the judicial sector. In 2003, we increased the independence of the judiciary by passing the Law on Court Budget, which created a separate court budget and Court Budget Council to administer the budgetary process. By mid-2005, we will provide additional staff to the administrative body of the Council. With the help of USAID, we will prepare a preliminary report on the financial costs of the judicial reform and complete a human resource needs assessment for the court administration and police for the 2006 budget (structural benchmark). We will also, by end-2006, complete - with the help of the European Agency for Reconstruction (EAR) - the process of introducing a new IT system in the judicial system.

\section{Other Measures to Improve the Business Climate}

\section{We will take the following measures to reduce obstacles to investment and} improve corporate governance:

- $\quad$ In order to ease the entry of new companies we will lighten procedures by concentrating all aspects of company registration at the Central Registry. Company registration will be moved from courts to the Central Registry by September 2005 and documentation requirements will be streamlined. By end-2005, the Central Registry and the PRO will agree on a procedure for the issuance of registration numbers that meets the administrative requirements of the PRO but also integrates the issuance of registration numbers at the Central Registry (structural benchmark).

- $\quad$ We will limit the number of licenses and streamline licensing procedures (thus reducing delays and the scope for corruption) in line with the WB's FIAS project.

- We will press ahead with a project (supported by the World Bank and USAID) to set up a reliable cadastre which will be finalized no later than 2009 .

- In order to improve corporate governance we will adopt by September 2005 a new Law on Audit that defines conditions for the audit of entities that perform activities in Macedonia. We have also made International Audit Standards binding and have published these standards in Macedonian. The establishment (by March-2006) of a self-governing institute for the qualification and certification of auditors will complete the public institution building process in this area. 
- $\quad$ Finally, exit procedures will be clarified and streamlined. By end-2005, bankruptcy procedures will be improved and accelerated with a planned revision of the Bankruptcy Law that strengthens creditors' rights and clarifies the role and the required qualifications of bankruptcy trustees (structural benchmark).

We intend to use the findings of the June 2005 corporate ROSC as a basis for developing and implementing further measures to improve the business environment.

\section{Financial Sector Strengthening}

\section{A. Central Bank Independence}

\section{The government will safeguard the independence of the NBRM by bolstering its} capital base and establishing a framework that will allow it to maintain a sufficient financial cushion over the medium term. The 2005 supplementary budget will restore the NBRM's capital to the level required by the central bank law through the issuance of marketable fixed income treasury securities paying interest on market-related terms. In addition, to make an up-front injection of reserve capital, the government will provide the NBRM with another 500 million denars of fixed income marketable government securities or cash. Later in 2005, in order to bolster the NBRM's ability to maintain a financial cushion, we will submit to parliament amendments to the central bank law to allow the NBRM to retain a large share of profits when general reserves are below a defined threshold and a smaller share when they are above the threshold. Passage of these amendments by parliament is a structural benchmark for the second review.

\section{B. Developing Financial Markets}

\section{In order to develop the market for treasury securities - which started operations} in 2004 - we are promoting secondary trading and the introduction of repurchase agreements. As a startup measure, the central securities depository and the NBRM have provided settlement and clearing services free of charge since April 2005. And, to enhance competition and increase transparency, information on securities prices was integrated with an electronic trading platform available to commercial banks in July 2005. Looking ahead, the NBRM and the Ministry of Finance will set the criteria for choosing market makers and sign a market maker agreement with major participants by end March 2006. The Ministry of Finance has carried out an educational campaign for banks and large non-bank treasury market participants on primary issuance as well as secondary treasury bill trading. Finally, to support the switch to open market operations in treasury bills, the NBRM has finalized all legal and accounting requirements for introducing repurchase agreements for banks and will start using repos for monetary interventions by end-September 2005 (structural benchmark). To this end, we are looking forward to IMF technical assistance (in July) on issues concerning repo transactions. 
24. The range of instruments for implementing monetary policy will be widened. We will take steps to reduce, and eventually end, the NBRM's reliance on central bank bills to absorb structural excess liquidity in the banking system. To that end, in the fall of 2005 the government and the NBRM will jointly present a plan to allow for the use of treasury bills for monetary policy purposes without creating an additional interest burden for the government budget (structural benchmark). Before that, and as a first step, the NBRM will start repo transactions with treasury securities obtained through the recapitalization operation. In addition, the NBRM will consider introducing a low-interest deposit facility, which will complete the interest rate corridor, thus providing a guide for market expectations of interest rates.

\section{The NBRM has also taken an active role in developing an inter-bank foreign} exchange market. The lack of real time information on transactions has limited the development of the market, resulting in large bid-ask spreads and an over-reliance on NBRM intervention. To address this problem, the NBRM has recently (June 2005) implemented an electronic system that provides electronic trading services and real time information on trades in foreign exchange. The NBRM has also signed a market maker agreement with the major participants in the foreign exchange market. The agreement sets limits on quote spreads and stipulates that the NBRM will deal only with these market makers.

\section{The NBRM will continue to improve the management of foreign exchange}

reserves. Since most of the NBRM's assets are in foreign reserves, its profitability depends heavily on income from these assets. In December 2004, the NBRM adopted new rules and investment guidelines - including the creation of an investment committee - on foreign exchange reserve management. Drawing on recent IMF technical assistance, the NBRM plans to improve the guidelines on the currency composition of reserves, the management of the benchmark portfolio and its intended maturities. The revised guidelines will come into effect in October 2005.

\section{Banking and Financial Sector Reforms}

\section{The NBRM and the government will take steps to improve the soundness of the} banking system. By end-2005, the Banking Law will be amended to: (i) improve governance by empowering the NBRM to revoke its approvals of bank shareholders no longer fulfilling the fit and proper criteria; (ii) improve the efficiency of the receivership process and tighten the criteria for bankruptcy trustees; (iii) Increase the minimum capital requirement for establishing a bank from Euro 3.5 million to Euro 5 million; (iv) harmonize the Banking Law with the Law on Trade Companies; (v) improve the provisions on banks' governance in line with the upcoming World Bank Corporate Governance Report; and (vi) introduce provisions for consumer protection. In order to strengthen credibility and accountability of the NBRM's supervision activities, we will prepare by end-2005 a Supervisory Development Plan for risk-based supervision based on a World Bank diagnostic mission in July 2005. Since strengthening of the financial sector is a core element of the program, the amendment of the Banking Law is a prior action for the second review, the development of the Supervisory Development Plan a structural benchmark. 
28. The NBRM will strengthen its capacity to assess and supervise credit, particularly foreign currency credit, and will provide appropriate credit-related information to the public on a regular basis. The supervision department has recently amended its manuals and regulations in order to ensure that banks' credit files contain the information needed to assess foreign exchange-induced credit risk. Prudential regulations will be revised and strengthened in order to limit exchange rate risk by end-2005. If necessary, follow up conditionality could become part of the second review. The revisions will draw on the recommendations of an upcoming IMF technical assistance mission on credit growth. In addition, from September 1, 2005 the NBRM will collect and publish more detailed interest rate and credit information in order to better follow credit developments. The publication will include an analysis of developments in loans indexed to or denominated in foreign exchange (in line with the publication in the 2004 annual report). Also by September 1, 2005, the NBRM will publish quarterly information on banks' soundness indicators.

\section{While credit growth has picked up, financial intermediation is still inefficient.}

The disincentives to bank lending include the high level of non-performing loans, weak contract enforcement, poor accounting, and limited information on credit performance. To strengthen bank intermediation, the NBRM and the government will: (i) enhance the information content of the NBRM's credit register, make financial and other data accessible for banks, and link the register with the central registry (by end-2006); (ii) issue accounting guidelines, a chart of accounts and formats for financial statements of banks in line with IAS based on the findings of the World Bank's REPARIS program and the work of the national steering committee on accounting and auditing by mid-2006; (iii) implement the law on data in Electronic Form, and E-Signature by end-2005.

\section{Also, by end-2005, the Law on Payment Operations and the law on the NBRM} will be amended in order to remove anomalies and clarify the NBRM's competencies. In line with the recommendations of the FSAP mission and IMF Technical Assistance mission, the Law on Payment Operations will be amended to: (i) remove provisions that allow holders of guarantees and bills of exchange to issue payment orders against the issuer's bank account; (ii) move procedural and operational issues from the law to manuals. In addition, by end-2005 we will submit to Parliament amendments to the Law on the NBRM to define more precisely its competences and actions, streamline the process for resolving appeals against the decisions and corrective measures that the NBRM imposes on banks.

\section{Public Sector Reforms}

\section{A. Budget Procedures and Transparency}

\section{We have made important strides in strengthening the transparency of the} budget process. Our recent introduction of program budgeting has clarified expenditure plans and laid the basis for better informed parliamentary debate. For the 2005 budget cycle, a number of reforms were enacted: (i) appropriations were incorporated according to budget 
programs, (ii) the content of the government's fiscal strategy paper was improved, (iii) line ministries were required to identify budget managers at each ministry, and (iv) expenditure limits were set early in the budgetary process. A side effect of the new budgetary process has been an increased emphasis on training.

\section{We now plan to take additional steps to improve budget planning and execution,}

as well as fiscal transparency. We will implement the following key measures:

- In July 2005 we will pass a new Budget Law that clarifies the procedures for preparation, adoption and execution of the budget. The new law will increase transparency by requiring the Ministry of Finance to prepare a mid-year budget report on both an economic and functional basis - with updated forecasts for the rest of the year. This report will be published on the Ministry of Finance's website.

- $\quad$ Starting with the 2006 budget, budget documents presented to Parliament will include a medium term fiscal framework (strategy) which will be used to assess medium-term policies, including public sector wages, employment, taxation, and spending priorities.

- $\quad$ During the 2006 budget process, budget management will be strengthened in key ministries - e.g., Agriculture, Education, Health, Justice and Transportation - through added training of the ministry staffs.

- The Ministry of Finance will continue to move toward GFS-consistent definitions in fiscal planning and reporting. Starting with the 2006 budget, the Ministry of Finance will use new GFS-consistent definitions, including local government budgets. Once the reporting framework for local governments has been established, the program will set fiscal targets at the general government level. In order to enhance transparency in the government's on-lending activities, the Ministry of Finance, with support from the NBRM's APEX unit database, will prepare, by June-2006, a report on on-lending by the central government to other government units and to the private sector (structural benchmark). Based on the findings of this report, steps will be taken to incorporate on-lending in the budget.

33. Public debt management and reporting will be strengthened. A fully staffed Public Debt Management Department has been created within the Ministry of Finance and has begun preparing a medium-term debt management strategy. Debt management software that meets specifications recommended by EAR and the IMF, will be selected by mid-2006. A public debt law was passed in July 2005 and a medium-term debt strategy will be submitted to parliament with the 2006 budget, including information on public debt sustainability, fiscal risks and the government's contingent liabilities. Fiscal transparency will be further enhanced through regular publication and analysis of statistics on public debt that will commence before end-2006. In line with program conditionality, we will limit nonconcessional debt to the limits spelled out in Table 1 and will not accumulate any public external arrears. 


\section{B. Tax Policy and Administration}

\section{In order to offset revenue losses caused by the ongoing trade liberalization} required by the WTO agreement, we plan to broaden the revenue base, including by cutting back tax incentive schemes. The loss of revenues caused by corporate tax incentives is estimated at around 1 billion denar per annum. The current revision of the corporate income tax law aims at eliminating incentives accounting for at least half of these losses by end-2005, but due to grandfathering rules revenues will only react starting in 2007 . In addition, no new free economic zone privileges will be granted and the existing ones will not be renewed. We will also broaden the personal income tax base by further measures to be defined in more detail at the time of the first review. A new tax will be levied in order to protect the environment, but with little impact on revenues.

\section{We plan to implement a comprehensive reform of tax administration in line with}

FAD recommendations. A new Law on Tax Administration Procedures was approved by government in July 2005 and will be passed by Parliament by end-2005 to expand taxpayers' protections and broaden the PRO's authorities. This will allow the PRO to impose penalties and seize assets without requiring prior court action. Organizational changes, rights and responsibilities of the PRO will be reflected in changes in the law on the PRO which will be sent to parliament together with the law on tax administration procedures. The PRO will also further strengthen its headquarters capacity in the areas of program development, operational planning, and support services by designing a new organization chart and filling new positions by end-2005 (structural performance criterion). Comprehensive programs will be developed for identifying and registering taxpayers, securing tax returns, recovering arrears, and detecting unreported taxes. Performance will be monitored by a comprehensive set of indicators for each tax administration function, which will be in place by January 2006. We will also conduct a national survey on taxpayer satisfaction to measure progress in taxpayer services. In order to meet the needs of different types of taxpayers, the PRO will establish a large taxpayer office (LTO) by July 2006 and a pilot office for administering small and medium taxpayers by July 2007. The PRO will develop a new computer system that is to be piloted at the LTO and compatible with the systems being developed by the social insurance funds by June 2006 (structural benchmark).

\section{To improve the collection of social insurance contributions, we will gradually} consolidate the collection activities of the pension, health, and unemployment funds and the PRO. By July 2006, we will create a new Large Contributor Office (LCO) comprising separate units for the PDF and the HIF and collecting contributions from the 100 largest employers (structural benchmark). By mid-2006 the government will approve the harmonization of the base across personal income tax and social contributions (structural performance criterion). By July 2008, the LCO will be merged with the LTO under the PRO to collect taxes and social contributions. At the same time we will decide on a time frame for merging fully the collection of PIT and social contributions. Donors have indicated their support for this ambitious reform project; the government will ensure that adequate budgetary resources are provided. In order to ensure speedy implementation of the planned 
reform which requires coordination of several government agencies and Extrabudgetary Funds, we have established an inter-ministerial steering committee to oversee and direct the reform (prior action).

\section{Governance in the Health Sector}

\section{We have taken steps to increase the efficiency, transparency, and fiscal}

soundness of the health care system. The government has approved legislative amendments in the Law on Health Insurance, the Law on Health Care Law, and changes in by-laws (including the HIF statute) that will strengthen the HIF Board and require transparent budget preparation, reporting and auditing by the HIF and Public Health Institutions (PHIs) (prior action). The revised amendments have been submitted to Parliament and are expected to be enacted by end-September, 2005. Specific measures, which have been prepared with technical assistance from the World Bank include the following:

- $\quad$ The amendments to the HIF statute reduce the number of HIF Board members to 7 and tighten the qualifications for membership. The new qualifications rule out conflict of interest and ensure that Board members have sufficient financial or accounting training to analyze the financial operations of the HIF. The amended law stipulates that representatives of the Ministries of Finance and Health will hold alternating chairmanship and vice-chairmanship of the Board and have a veto over Board decisions.

- $\quad$ The approved amendments to the Laws on Health Insurance and Health Care (and associated by-laws) create a legal framework for transparent budget preparation (in line with the HIF's action plan) and for reporting by the HIF and PHIs in a format mandated by $\mathrm{MOH}$ and MOF. Specific features include binding spending ceilings for PHIs and the abolition of the HIF's compulsory reserve.

- $\quad$ Starting in May 2005, monthly HIF budget execution reports have been published on the HIF website. The reports provide details of HIF transfers to PHIs (by institution) and of PHIs' expenditures in economic classification (on both cash and commitment bases). They also include monthly financial reports in functional classification for the largest 7 PHIs (on both cash and a commitment bases). Starting with the 2006 budget, the HIF will report on budget execution, including transfers to PHI's, in functional classification, on a cash and commitment basis (structural benchmark). By mid-2006, reporting in economic classification will be added (structural benchmark). Reports on functional classification (on both cash and commitment bases) will be included for all PHIs starting in mid-2006.

- The 7 largest PHIs will prepare budgets for the 2006 fiscal year, for approval by the $\mathrm{MOH}$ in consultation with the HIF and the MOF. Once approved, the PHI budgets, which need to be in line with the HIF's transfers, will impose binding spending ceilings (structural performance criterion). For the 2007 fiscal year and thereafter budgets will be prepared for all PHIs. 
- We will publish external audits (including reactions and comments) of the financial statements of the 7 largest health care institutions by mid-2007. Audits of the remaining health institutions will be published by mid-2008.

\section{There will be no further accumulation of arrears by the HIF (performance} criterion) and the PHIs, and steps will be taken to address the existing stock of arrears. HIF arrears will be inventoried and audited by mid-2006 and HIF budgets will make allowance for the repayment of arrears over 2007-08. All future purchase contracts will include a clause that prohibits factoring of the resulting receivables. The Ministry of Finance's Central Internal Audit Department will ensure that, in line with the HIF law, no further financial operations which loosen the HIF's budget constraint, are undertaken.

\section{Other Public Sector Reforms}

\section{The newly constituted municipalities — a key element of the Framework} Agreement-begun operations in June 2005, posing significant challenges in the areas of monitoring local government finances and clearing inherited municipal arrears. Monitoring will be facilitated by a unified treasury: in June all municipalities closed their commercial bank accounts and moved their funds into the government's treasury single account (TSA). Resolving municipal arrears, which were inherited from local governments that no longer exist, poses complex problems. The government will adopt (by September 2005) a debt resolution plan that balances creditors' rights, municipalities' ability to pay, and moral hazard considerations. The terms of the debt resolution plan will be decided through government-facilitated negotiations between municipalities and creditors. The government will cover a limited part of the debt (in addition to claims arising from expropriation) in order to help mobilize burden-sharing by donors and creditors. To prevent new arrears while ensuring high quality local services, budget preparation and execution procedures, including commitment control, will be introduced in all municipalities by end-2005. Borrowing by the municipalities will be limited during the first two years in line with the Law on Local Government Finance and the government will not approve loans unless they are on concessional terms and the municipality is financially sound.

40. In January 2006 we will introduce a second pillar pension program which is intended to help put the public pension system on a sustainable path. The reform, which is the culmination of several years' planning, has benefited from technical support by USAID and the World Bank. Since this reform will require major administrative changes, the PDF plans to implement a 3 month pilot phase before bringing the system into operation.

\section{E. Public Sector Management and Governance}

41. Key ministries will be rationalized and reorganized in line with the advice of the World Bank, EAR, DFID. By end-December we will complete the functional analyses of ministries that is being undertaken with support from DFID (structural benchmark). The findings of these analyses will be presented by individual ministries in their 2007 budget 
submissions to the MOF by mid-2006 (structural benchmark). The management of line ministries will be enhanced by institutional reforms that emphasize improved budgeting, merit-based selection and promotion policies, and changes in the organizational structure to improve the linkage between resources and responsibilities. Given the scale of future changes in the composition and capacity of the public sector, we also intend to draw up a medium term plan for public employment.

\section{We will sell the state's minority shares in private enterprises and work to} improve transparency in the management of the remaining state assets. In addition to the large privatization projects (see $\$ 8$ above), the Government will sell the equity shares held by the PDF in private companies. In order to enhance transparency in the management and privatization of state assets, the Government will report to Parliament on an annual basis on the remaining public companies and on the state's equity shares in private companies (structural benchmark). The reports will provide basic financial information, list the government's representatives in these companies and describe plans for privatization. In addition, the Law on Public Enterprises will be amended to enhance efficiency and transparency, including by (i) introducing IAS for financial statements of public enterprises, (ii) moving the external audit from the state audit office to a private auditor, and (iii) making the management of public enterprises compliant with EU directives.

\section{By mid-2005, we will strengthen the assessment and audit of income disclosure} reports of state officials. While the law on corruption includes a comprehensive reporting requirement for government officials, these reports are not yet consistently analyzed and audited. The anti-corruption commission will be empowered to audit randomly selected disclosure reports of senior elected and appointed officials and civil servants (structural benchmark).

\section{Statistics}

44. We will undertake measures to improve national statistics. As statistical data is essential for effective economic surveillance, policy management, and FDI, the State Statistic Office (SSO) will further improve its work. With the help of IMF technical assistance, the SSO has produced a work plan to develop quarterly national accounts. Quarterly national account series should be ready by end-2005. Moreover, we will request additional technical assistance from the EAR and ILO in the area of price, employment, and national accounts statistics.

\section{Program Monitoring}

45. The program will be reviewed by the IMF semiannually, starting with a first review based on end-December data. The first, second and third review will be completed by February 15, 2006, August 15, 2006 and February 15, 2007.

46. Prior actions as well as performance criteria and indicative targets have been set for the first 12 months of the program. Quantitative performance criteria and indicative 
targets, consistent with our intention to maintain contain government borrowing and increase international reserves, are set out in Table 1. Structural conditionality, which focuses on our reforms in the areas of tax administration, public expenditure management (notably in the health sector), and the business climate (labor market and court reforms), are set out in Table 2 .

47. Conditionality for the second and third year of the arrangement will be set in the context of program reviews. Macroeconomic conditionality will be anchored by the need to achieve the fiscal deficit and gross reserves targets set out in Section II and in the Annex of this MEFP. Structural conditionality will focus on bringing the reform of payroll tax collection to conclusion ( $\$ 36$ ), further improvements in health sector efficiency ( $\$ 37$ and 38), institutional changes needed for the judicial reform ( $\mid 15-20)$, improvements in bank supervision and prudential regulation ( ( 27-30) and in public sector management (\$ 41-43). 


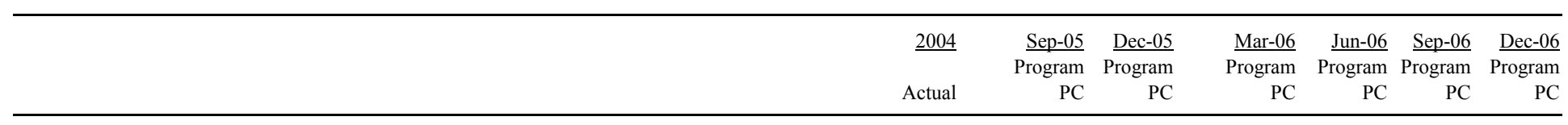

Floor for net international reserves of the NBRM

Reserve assets *

Reserve liabilities **

End-year stocks

613
717
104

Ceiling on new nonconcessional medium- and long-term external debt contracted or guaranteed by the general government or the NBRM with original maturities of more than 1 year

Ceiling on short-term external debt of the central government or the NBRM with maturities of up to 1 year (stock); including guarantees for such debt

Accumulation of external payments arrears

\section{Ceiling on net domestic assets of the NBRM}

Ceiling on net domestic assets of the banking system (indicative target)

Ceiling on net domestic credit to the central government from the NBRM

Ceiling on Health Insurance Fund's arrears

Ceiling on central government's total domestic arrears (indicative target) Memo item: Non HIF domestic arrears

Central government wages (indicative target)

Floor for central government fiscal balance (in millions of euros)

Cumulative changes since end-Dec. 2004

$\begin{array}{rrrrrr}28 & 176 & 296 & 305 & 339 & 345 \\ 55 & 199 & 320 & 325 & 359 & 361\end{array}$

$\begin{array}{lllllll}\cdots & 0 & 0 & 0 & 0 & 0 & 0 \\ 0 & 0 & 0 & 0 & 0 & 0 & 0\end{array}$

Sources: Data provided by the authorities; and IMF staff estimates.

* Quarterly flows for 2005 revised to reflect that the Telecom dividend to foreigners will be paid in the 3rd quarter of 2005 instead of in the 2nd

** Actuals for Q1-05 and subsequently increase by the increase of net liabilities to the IMF. In line with the NBRM information, the NIR PCs

assume no change in pledged foreign assets during the program period.
End-year stocks

$-20,713$

25,916

$-8,075$

1,710

2,019

Annual flows

22,032 (in millions of denars)

Cumulative changes since end-Dec. 2004

(in millions of denars)

Cumulative flows within year 


\section{Prior Actions}

1. Government approval of the 2005 supplementary budget (MEFP, 10)

2. Passage by Parliament of a new Law on Labor Relations (MEFP, 14).

3. Government submission to Parliament of amendments to the Health Insurance Law and Law on Health Care and adoption of

the necessary by-laws to tighten selection of HIF Board and implement transparent budget procedures (MEFP, 37).

4. Government to establish an interministerial steering committee with authority to implement the planned reform of payroll tax collection (MEFP, 36).

\section{First Review}

Performance Criteria

1. The 7 largest PHI's prepare 2006 budgets, approved by the MOH, that are in line with the HIF transfers, and become binding ceilings (MEFP, 37).

2. PRO designs new organizational chart and fills identified positions required to implement payroll tax reform (MEFP, 35).

3. Parliament to enact a 2006 budget that is in line with the program (MEFP, 6).

4. Government to submit new Banking Law to Parliament (MEFP, 27)

Benchmarks

1. Judicial Budget Council to prepare 2006 court budget, including an analysis of the fiscal implications of judicial reform (MEFP, 20).

2. Amend the Law on the Chamber of Commerce to allow existing members of employers' associations to terminate their membership prior to the negotiation of the next collective agreements (MEFP, 14).

3. Remove company registration from courts and establish an integrated company numbering system at the Central Registry (MEFP, 21).

4. Parliament approves amendments to the Bankruptcy Law (MEFP, 21).

5. MOF will start tendering for the sale of residual state shares in commercial banks (MEFP, 8).

6. Government and NBRM to agree on a plan for the use of treasury bills for monetary policy purposes (MEFP, 24).

7. NBRM will start using repo transactions for monetary interventions (MEFP, 23).

8. NBRM to prepare a Supervisory Development Plan for risk-based supervision (MEFP, 27).

9. HIF to prepare a 2006 budget and to start monthly reporting on budget execution, including transfers to the PHI's in functional classification, on a cash and commitment basis (MEFP, 37).

10. The government to finalize the functional analysis of line ministries and to prepare a plan for its implementation (MEFP, 41).

By end-2005

By end-2005

By end-2005

By end-2005

By end-2005

By end-2005

By end-2005

By end-2005

By end-2005

By end-2005

By end-September 2005

By end-2005

By end-2005

By end-200

By July 2006

By March/June 2006

By June 2006

By June 2006

By June 2006

By June 2006

By June 2006

By July 2006

By June 2006

By June 2006

By June 2006

3. Prepare a report on the government's economic activities (MEFP, 42)

4. Anti-corruption commission to audit the financial disclosure reports of randomly chosen senior elected and appointed officials and civil servants (MEFP, 43) sanctions on misdemeanor cases without prior court involvement (MEFP, 19).

6. HIF to report on its spending in economic classification, on a cash and commitment basis, including a breakdown of the PHI's spending, based on the Q1 2006 outcome (MEFP, 37).

7. Pass amendments to the NBRM law that revise provisions on the retention/distribution of NBRM profits (MEFP, 22).

8. The systematization for all line ministries will be revised based on the findings of a functional analysis and will be reflected in the 2007 budget (MEFP, 41 ). 
Table 3. Summary and Timetable for Structural Measures

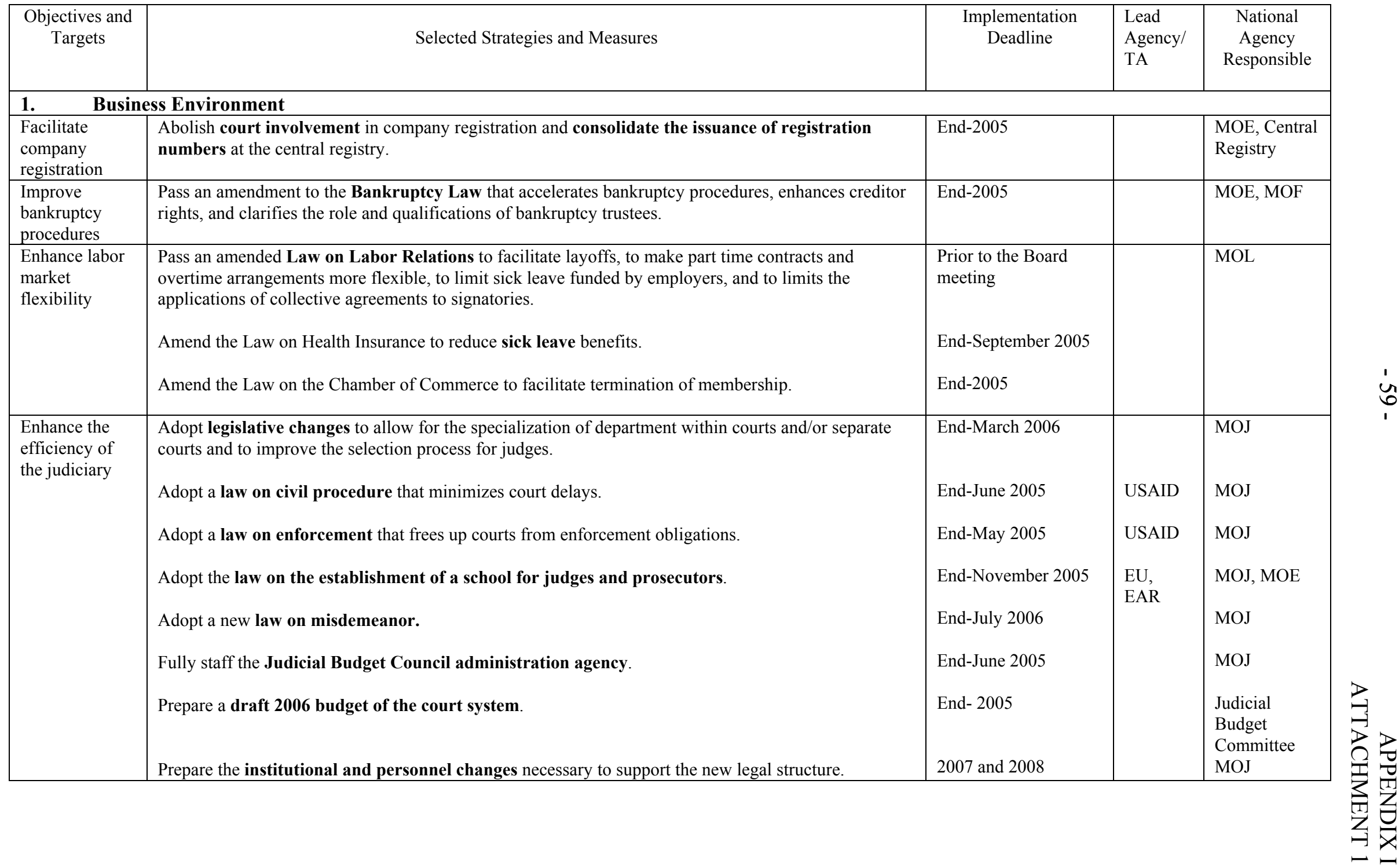




\begin{tabular}{|c|c|c|c|c|}
\hline \multicolumn{5}{|c|}{ 2. Financial Sector Reform } \\
\hline $\begin{array}{l}\text { Improve the } \\
\text { soundness of } \\
\text { the financial } \\
\text { system }\end{array}$ & $\begin{array}{l}\text { Submit to parliament an amendment to the Banking Law, including enhanced regulations on fit and } \\
\text { proper criteria, withdrawal of licenses, and on receivership. } \\
\text { Enhance the payment system by amending the Law on Payment Operations. Amend the Law on the } \\
\text { NBRM to clarify the NBRM's competencies. } \\
\text { Enhance banking supervision, including by } \\
\text { (i) reassessing prudential regulations related to the exchange rate risk in bank's credit portfolio based on } \\
\text { the findings of the upcoming IMF TA on credit growth, } \\
\text { (ii) strengthening the analysis and publication of trends in banks' lending and interest activities, } \\
\text { (iii) preparing a Supervisory Development Plan for risk-based supervision. }\end{array}$ & $\begin{array}{l}\text { End-2005 } \\
\text { End-September } 2005 \\
\text { End-2005 }\end{array}$ & $\begin{array}{l}\text { IMF } \\
\text { FSAP }\end{array}$ & NBRM, MOF \\
\hline $\begin{array}{l}\text { Strengthen } \\
\text { financial } \\
\text { intermediation }\end{array}$ & Render the upgraded NBRM credit register fully operational and linked with the central registry. & End-2006 & & $\begin{array}{l}\text { NBRM, } \\
\text { Central } \\
\text { Registry }\end{array}$ \\
\hline $\begin{array}{l}\text { International } \\
\text { reserves } \\
\text { management }\end{array}$ & $\begin{array}{l}\text { Improve the guidelines on currency composition and maturity of reserves, and on the management of } \\
\text { benchmark portfolios in line with MFD recommendations. }\end{array}$ & End-October 2005 & IMF & NBRM \\
\hline $\begin{array}{l}\text { Develop the } \\
\text { secondary } \\
\text { market for } \\
\text { treasury bills }\end{array}$ & $\begin{array}{l}\text { Establish a framework for secondary trading of treasury bills by setting up appropriate trading and } \\
\text { information systems. } \\
\text { Launch repo transactions for market participants. } \\
\text { Start monetary interventions through repo transactions. } \\
\text { Develop a plan for the use of treasury bills for monetary policy purposes. }\end{array}$ & $\begin{array}{l}\text { End-July } 2005 \\
\text { End-July } 2005 \\
\text { End-September } 2005 \\
\text { End-2005 }\end{array}$ & $\begin{array}{l}\text { IMF } \\
\text { IMF }\end{array}$ & $\begin{array}{l}\text { NBRM and } \\
\text { MOF }\end{array}$ \\
\hline $\begin{array}{l}\text { Recapitalize } \\
\text { the NBRM }\end{array}$ & $\begin{array}{l}\text { Include the recapitalization of the NBRM in the supplementary budget. } \\
\text { Amend the NBRM Law to increase the NBRM's share in the profit allocation. }\end{array}$ & $\begin{array}{l}\text { End-September } 2005 \\
\text { End-June } 2006\end{array}$ & & MOF \\
\hline 3. $\quad$ Fisca & Sector Efficiency & & & \\
\hline $\begin{array}{l}\text { Enhance } \\
\text { budget } \\
\text { planning and } \\
\text { execution; } \\
\text { improve fiscal } \\
\text { transparency }\end{array}$ & $\begin{array}{l}\text { Pass the new Budget Law. } \\
\text { Strengthen budget preparation and execution in the municipalities; enhance monitoring of } \\
\text { municipal financing. } \\
\text { Move toward fully GFS-consistent definitions in fiscal planning and reporting. } \\
\text { Develop a rolling medium-term fiscal framework (fiscal strategy). }\end{array}$ & $\begin{array}{l}\text { End-July } 2005 \\
\text { End-2005 } \\
\text { For the } 2006 \text { budget } \\
\text { process }\end{array}$ & $\begin{array}{l}\text { US } \\
\text { Treasury }\end{array}$ & MOF \\
\hline
\end{tabular}




\begin{tabular}{|c|c|c|c|c|}
\hline & Publish data on the government's on-lending activities. & End-June 2006 & & \\
\hline $\begin{array}{l}\text { Broaden the tax } \\
\text { base }\end{array}$ & $\begin{array}{l}\text { Abolish CIT incentive schemes accounting for at least half of the fiscal costs. } \\
\text { Stop granting new free economic zone privileges. }\end{array}$ & $\begin{array}{l}\text { End-2005 } \\
\text { August } 2005\end{array}$ & & MOF, PR \\
\hline $\begin{array}{l}\text { Enhance the } \\
\text { effectiveness of } \\
\text { the PRO }\end{array}$ & $\begin{array}{l}\text { Submit a Law on Tax Administration and a Law on the PRO to parliament. } \\
\text { Improve tax administration in line with FAD recommendations: } \\
\text { (i) Strengthen PRO headquarters by developing a new organizational chart and by filling } \\
\text { identified vacant positions. } \\
\text { (ii) } \\
\text { Formulate a comprehensive set of performance measures for tax administration functions. } \\
\text { (iii) Establishing a large taxpayer office. } \\
\text { (iv) Set up a pilot office for administering small and medium taxpayers. } \\
\text { (v) Test a new computer system compatible with social funds' systems. }\end{array}$ & $\begin{array}{l}\text { End-July } 2005 \\
\text { End-December } 2005 \\
\text { June } 2006 \\
\text { End-July } 2006 \\
\text { July } 2007 / \text { End-July } \\
2006\end{array}$ & IMF & MOF, PDF \\
\hline $\begin{array}{l}\text { Coordinate and } \\
\text { merge the } \\
\text { collection of } \\
\text { taxes and social } \\
\text { contributions }\end{array}$ & $\begin{array}{l}\text { Establish an interministerial committee with the authority to consolidate social contribution collection } \\
\text { and PIT. } \\
\text { Establish a large contributor office comprising the PDF and the HIF. } \\
\text { Adopt harmonized bases for PIT and social insurance contribution. } \\
\text { Merge LCO with LTO and decide on a timeframe for the full merger of PIT and social contribution } \\
\text { collection. }\end{array}$ & $\begin{array}{l}\text { Prior to the Board } \\
\text { Meeting } \\
\text { End-July } 2006 \\
\text { End-July } 2006 \\
\text { July } 2008\end{array}$ & IMF & $\begin{array}{l}\text { MOF, MOL, } \\
\text { MOH }\end{array}$ \\
\hline $\begin{array}{l}\text { Strengthen debt } \\
\text { management } \\
\text { capacity }\end{array}$ & $\begin{array}{l}\text { Pass a Public Debt Law. } \\
\text { Present a medium-term debt strategy and report regularly on debt developments. } \\
\text { Prohibit borrowing by local governments for the first } 2 \text { years after the law on local government } \\
\text { finances becomes effective. }\end{array}$ & $\begin{array}{l}\text { End-July } 2005 \\
\text { For the } 2006 \text { budget } \\
\text { Continuous }\end{array}$ & EAR & MOF \\
\hline $\begin{array}{l}\text { Resolve the } \\
\text { municipal } \\
\text { arrears }\end{array}$ & Adopt a plan to clear or reschedule municipal arrears. & September 2005 & $\begin{array}{l}\text { USAID, } \\
\text { EU }\end{array}$ & MOF \\
\hline $4 . \quad$ Healt & Sector Governance & & & \\
\hline $\begin{array}{l}\text { Improve } \\
\text { governance in } \\
\text { the health } \\
\text { sector }\end{array}$ & $\begin{array}{l}\text { Government approval on amendments in the Health Insurance Law and the Law on Health Care to } \\
\text { tighten the selection criteria for HIF board members and to enhance budget preparation and } \\
\text { reporting in Public Health Institutions (PHIs) } \\
\text { HIF to publish monthly information on budget execution, including an analysis of transfers to the } \\
\text { PHIs. } \\
\text { Report on the HIF website for at least two months on revenues and expenditure of the largest } 7 \text { PHIs. }\end{array}$ & $\begin{array}{l}\text { Prior to the IMF } \\
\text { Board meeting } \\
\text { End-June } 2005 \\
\text { End-June } 2005\end{array}$ & $\begin{array}{l}\text { IMF and } \\
\text { World } \\
\text { Bank }\end{array}$ & $\mathrm{MOH}, \mathrm{MOF}$ \\
\hline
\end{tabular}




\begin{tabular}{|c|c|c|c|c|}
\hline & $\begin{array}{l}\text { Prepare budgets for } 2006 \text { for the } 7 \text { largest PHIs. } \\
\text { All remaining PHIs to follow the reporting and budgeting procedures described above. } \\
\text { HIF to report on budget execution in economic classification, including reporting on PHI's. } \\
\text { Undertake audits for the } 7 \text { largest PHIs and publish the results. } \\
\text { Undertake audits of the remaining PHIs. }\end{array}$ & $\begin{array}{l}\text { For the } 2006 \text { budget } \\
\text { End-June } 2006 / \\
\text { For the } 2007 \text { budget } \\
\text { End-June } 2006 \\
\text { Mid-2007 } \\
\text { Mid-2008 }\end{array}$ & & \\
\hline \multicolumn{5}{|c|}{ Public Sector Management and Governance } \\
\hline $\begin{array}{l}\text { Reduce the risk } \\
\text { of conflict of } \\
\text { interest }\end{array}$ & $\begin{array}{l}\text { Strengthen the anti-corruption commission's capacity to assess and audit randomly state official's } \\
\text { income disclosures statements. }\end{array}$ & End-June 2006 & & $\mathrm{MOL}$ \\
\hline $\begin{array}{l}\text { Enhance } \\
\text { transparency of } \\
\text { state owned } \\
\text { enterprises }\end{array}$ & $\begin{array}{l}\text { Amend the law on public enterprises. } \\
\text { Prepare annual reports on the state's economic activities. }\end{array}$ & $\begin{array}{l}\text { End-2005 } \\
\text { End-2006 }\end{array}$ & IMF & MOF \\
\hline $\begin{array}{l}\text { Improve the } \\
\text { efficiency of } \\
\text { line ministries }\end{array}$ & $\begin{array}{l}\text { Reorganize key ministries in line with the findings of the functional analysis. } \\
\text { Develop a plan for public employment. }\end{array}$ & For the 2007 budget & & \\
\hline
\end{tabular}

Abbreviations used in the text:

$\begin{array}{llll}\text { CIT } & \text { Corporate Income Tax } & \text { MOJ } & \text { Ministry of Justice } \\ \text { DFID } & \text { Department for International Development } & \text { MOL } & \text { Ministry of Labor } \\ \text { EAR } & \text { European Agency for Reconstruction } & \text { NBRM } & \text { National Bank of the Republic of Macedonia } \\ \text { EU } & \text { European Union } & \text { PDF } & \text { Pension and Disability Fund } \\ \text { EF } & \text { Employment Fund } & \text { PHI } & \text { Public Health Institution } \\ \text { FAD } & \text { IMF Fiscal Affairs Department } & \text { PIT } & \text { Personal Income Tax } \\ \text { HIF } & \text { Health Insurance Fund } & \text { PRO } & \text { Public Revenue Office } \\ \text { IAS } & \text { International Accounting Standards } & \text { TA } & \text { Technical Assistance } \\ \text { MOF } & \text { Ministry of Finance } & \text { USAID } & \text { US Agency for International Development } \\ \text { MOE } & \text { Ministry of Economy } & \text { VAT } & \text { Value Added Tax } \\ \text { MOH } & \text { Ministry of Health } & \text { WB } & \text { World Bank } \\ \text { MOT } & \text { Ministry of Transportation } & & \end{array}$




\section{TeChNical Memorandum of Understanding}

This Technical Memorandum of Understanding (TMU) defines the variables subject to quantitative targets (performance criteria and indicative benchmarks), established in the Memorandum of Economic and Financial Policies (MEFP) and describes the methods to be used in assessing the program performance with respect to these targets.

\section{A. Government and Public Sector}

1. For the purpose of this TMU, the term "central government" covers: central government as defined in the Annual Budget Document, (including Special Revenue Accounts), Employment Fund, Health Insurance Fund, Pension Insurance Fund, Road Fund, and agencies and institutions that are currently treated by the Ministry of Finance as part of government and which correspond to the classification followed by the National Bank of the Republic of Macedonia (NBRM) in its monthly submissions to the Fund of balance sheets of the central bank and the consolidated accounts of the commercial banks. The authorities will inform the Fund staff of any new funds, or other special budgetary and extrabudgetary programs that may be created during the program period to carry out operations of a fiscal nature as defined in the IMF's Manual on Government Financial Statistics, and will ensure that these will be incorporated within the definition of consolidated central government.

2. The term "general government" covers the central government as defined in $\llbracket 1$ and the municipalities which are classified as part of general government according the budget documents and which are included by the National Bank of the Republic of Macedonia (NBRM) in its monthly submissions to the Fund of balance sheets of the central bank and the consolidated accounts of the commercial banks.

3. The term "public sector" covers the general government as defined in $₫ 2$ and foreign financed projects of public enterprises.

\section{B. Net International Reserves of the NBRM}

4. Net international reserves (NIR) of the NBRM are defined as the difference between NBRM's reserve assets and its reserve liabilities.

5. Reserve assets are defined as liquid and usable foreign convertible currency claims on nonresidents plus monetary gold. Reserve assets of the NBRM thus include monetary gold, SDRs, foreign currency cash, securities, deposits abroad, and the reserve position at the Fund. Excluded from reserve assets are any assets that are frozen, pledged, used as collateral, or otherwise encumbered, claims in foreign exchange arising from transactions in derivative assets (futures, forwards, swaps, and options), and precious metals other than gold.

6. Reserve liabilities are defined as all foreign exchange liabilities of the NBRM to nonresidents and residents, including all credit outstanding from the Fund, arrears on 
principal or interest payments to commercial banks, suppliers, or official export credit agencies, and future and contingent commitments to sell foreign exchange arising from transactions in derivative assets (futures, forwards, swaps, and options). Central government's foreign exchange deposits at the NBRM are excluded from reserve liabilities.

7. For program purposes, all foreign currency-related assets and liabilities are valued at program exchange rates as defined in Section $\mathrm{J}$.

8. At end-December 2004, reserve assets so defined stood at EUR 717.0 million; reserve liabilities so defined stood at EUR 104.0 million; and NIR so defined stood at EUR 613.0 million (Table 1).

9. Semi-annual floors (NIR floors) have been established for the cumulative changes in the NIR of the NBRM from the level at end-December 2004 (Table 1 of the MEFP). The changes in the NIR will be measured in euros excluding valuation effects calculated according to the methodology described in Section $\mathrm{J}$.

\section{Adjustors}

10. The NIR floors are set based on the assumption that balance of payments financing will amount on a cumulative basis, from end-December 2004 to:

End-September 2005

End-December 2005

End-March 2006

End-June 2006

End-September 2006

End-December 2006
EUR 32.3 million;

EUR 231.3 million;

EUR 372.5million;

EUR 372.5 million;

EUR 372.5 million;

EUR 394.5 million.

11. Balance of payments financing is defined as the sum of: i) gross disbursement of foreign loans or grants to the central government or the NBRM for balance of payments financing, including the planned issuance of Eurobonds, minus the planned prepayment of foreign loans; ii) privatization proceeds and lump sum proceeds from concession fees in foreign currency; and, iii) proceeds from the restitution of foreign assets of the former SFRY. Project loans and grants, and purchases from the IMF are not considered balance of payments financing for this purpose.

12. If balance of payments financing deviates from the baseline path shown above and in Table 2, the NIR floors of the NBRM will be adjusted as follows:

- $\quad$ The NIR floor will be adjusted upward (downward) to the same extent as any upward (downward) deviation in grants and loan disbursements from bilateral and multilateral donors, with the provision that the downward adjustment to the floor will not exceed the equivalent of EUR 30 million on a cumulative basis. 
- In the event that the proceeds from the Eurobond exceed EUR 150 million, the NIR floors will be adjusted upward by the amount of the excess. In the event that the proceeds from the Eurobond issue fall short of EUR 150 million but exceed EUR 130 million, the NIR floors will remain unchanged. In the event that the proceeds from the Eurobond issue fall short of EUR 130 million, the NIR floors will be adjusted downward by the amount of the shortfall.

- $\quad$ The NIR floors will be adjusted upward (downward) by the amount of any prepayment of external debt falling short of (exceeding) the baseline defined in Table 2.

- $\quad$ The NIR floors will be adjusted upward (downward) for any privatization proceeds or lump sum proceeds from concession fees in foreign currency exceeding (falling short of) the baseline defined in Table 2, with the provision that the downward adjustment to the floor will not exceed the equivalent of EUR 50 million on a cumulative basis.

- $\quad$ The NIR floors will be adjusted upward (downward) for the restitution of foreign assets of the former SFRY as a result of succession proceeds exceeding (falling short) of the baseline defined in Table 2 .

- $\quad$ The sum of upward and downward adjustments to NIR floor in response to deviations from the baseline balance of payment financing projections will not result in a net downward adjustment exceeding the following limits:

End-September 2005

End-December 2005

End-March 2006

End-June 2006

End-September 2006

End-December 2006
EUR 30.0 million;

EUR 166.5 million;

EUR 81.5 million;

EUR 76.5 million;

EUR 71.5 million;

EUR 66.5 million.

\section{Net Domestic Assets of the NBRM}

13. Net domestic assets (NDA) of the NBRM are defined as reserve money minus the net foreign assets (NFA) of the NBRM.

14. Reserve money is defined as currency in circulation (outside banks), vault cash of banks, and required and excess reserve deposits of banks in denars and in foreign currency held at the NBRM or at the NBRM accounts abroad.

15. Net foreign assets (NFA) of the NBRM are defined as reserve assets plus those foreign assets of the NBRM that are excluded from reserve assets under the definition in 15 of this TMU, minus foreign exchange liabilities of the NBRM to nonresidents. 
16. At end-December 2004, reserve money so defined stood at denar 21,111 million; NFA so defined stood at EUR 682.2 million or denar 41,824 million (converted using the exchange rate methodology described in Section J); and NDA so defined stood at denar 20,713 million.

17. Semi-annual ceilings (ceilings for NDA) have been established for the cumulative changes in the NDA of the NBRM from the level at end-December 2004 (Table 1 of the MEFP).

\section{Adjustors}

18. If balance of payments financing deviates from the baseline path shown in Table 2, the ceilings for the NDA of the NBRM will be adjusted as follows:

- $\quad$ The NDA ceilings will be adjusted downward (upward) to the same extent as any upward (downward) deviation in grants and loan disbursements from bilateral and multilateral donors, with the provision that the upward adjustment to the floor will not exceed the equivalent of EUR 30 million on a cumulative basis.

- In the event that the proceeds from the Eurobond exceed EUR 150 million, the NDA ceilings will be adjusted downward by the amount of the excess. In the event that the proceeds from the Eurobond issue fall short of EUR 150 million but exceed EUR 130 million, the NDA ceilings will remain unchanged. In the event that the proceeds from the Eurobond issue fall short of EUR 130 million, the NDA ceilings will be adjusted upward by the shortfall.

- $\quad$ The NDA ceiling will be adjusted downward (upward) by the amount of any prepayment of external debt falling short of (exceeding) the baseline defined in Table 2.

- $\quad$ The ceilings for the NDA of the NBRM will be adjusted downward (upward) for any privatization proceeds or lump sum proceeds from concession fees in foreign currency exceeding (falling short of) the baseline defined in Table 2, with the provision that the upward adjustment to the ceiling will not exceed the equivalent of EUR 50 million on a cumulative basis.

- The ceilings for the NDA of the NBRM will be adjusted downward (upward) for the restitution of foreign assets of the former SFRY as a result of succession proceeds exceeding (falling short) of the baseline defined in Table 2. 


\section{Net Domestic Assets of the Banking System}

19. Net domestic assets (NDA) of the banking system, which includes the NBRM and the deposit money banks, are defined as broad money (M3) minus the net foreign assets (NFA) of the banking system.

20. Broad money (M3) includes currency in circulation, demand deposits, quasideposits, and non-monetary deposits (time deposits over 12 months and restricted deposits) of the non-central government denominated in denar and in foreign currency.

21. NFA of the banking system are defined as the banking system's foreign assets minus foreign liabilities.

22. At end-December 2004, broad money so defined stood at denar 94,781 million; NFA of the banking system so defined stood at denar 68,865 million; and NDA of the banking system so defined stood at denar 25,916 million.

23. Semi-annual indicative ceilings have been established for the cumulative changes in the NDA of the banking system from the level at end-December 2004 (Table 1 of the MEFP).

\section{Adjustors}

24. The ceilings on the NDA of the banking system will be subject to the same adjustors as the ceilings on the NDA of the NBRM.

\section{E. Net Credit to the Central Government from the NBRM}

25. Net credit to the central government from the NBRM system is defined as credit in denar and foreign currency to the central government from the NBRM minus total central government deposits in denar and foreign currency with the NBRM. For the purpose of this program, accounts of the central government include all accounts recorded as central government accounts in the monetary statistics reported by the NBRM in accordance with the definition of central government $\$ 1$. Excluded from this definition are any T-bills issued for monetary policy purposes and corresponding government deposits at the NBRM, and the unclaimed portion of the payment of principal and interest on frozen foreign currency deposits.

26. At end-December 2004, the amount of outstanding credit from the NBRM to the central government in denar and foreign currency stood at denar 2,881 million; the amount of central government deposits held at the NBRM was equal to denar 10,956 million (excluding denar 1743 million corresponding to the unclaimed portion of the payment of principal and interest on frozen foreign currency deposits which is recorded as Other Items Net). In sum, net credit of the NBRM to the central government so defined stood at denar $-8,075$ million. 
27. Semi-annual ceilings have been established for the cumulative changes in net credit from the NBRM to the central government from the level at end-December 2004 (Table 1 of the MEFP).

\section{Adjustors}

28. The ceilings on net credit to the central government from the NBRM will be subject to the same adjustors as the ceilings for the NDA of the NBRM. With the exception that if the proceeds from the Eurobond issue fall short of EUR 150 million but exceed EUR 130 million, the ceiling for the Net Credit to the Central Government from the NBRM will be adjusted upward by one half of the amount of the shortfall. (Given that the NIR floor would remain unchanged in the event that the proceeds from the Eurobond issue fall short of EUR 150 million but exceed EUR 130 million, this adjustor would ensure that the adjustment needed to meet the NIR target would be shared equally between the NIRM and the government.) In the event that the proceeds from the Eurobond issue fall short of EUR 130 million, the ceiling for Net Credit to the Central Government from the NBRM will be adjusted upward by the amount that the proceeds fall short of EUR130 million plus EUR 10 million.

\section{F. Government Fiscal Balances}

29. Semi-annual floors for the cumulative changes in central government fiscal balances will be determined and monitored from the financing side beginning end-December 2004 (Table 1 of the MEFP). The financing flows will be measured as a sum of domestic financing, foreign financing, and privatization proceeds.

30. Domestic financing for the central government includes net credit from the domestic banking system (excluding any T-bills issued for monetary policy purposes and corresponding government deposits at the NBRM, and the unclaimed portion of the repayment of frozen foreign currency deposits), net placement of securities outside the domestic banking system and other net credit from the domestic non-banking sector, and net variation in domestic arrears. Foreign financing for the central government (converted using the valuation methodology described in Section J) includes disbursements of external loans received by the central government, including disbursements received for foreign financed projects of budget users and extrabudgetary funds, and restitution of foreign assets of the former SFRY as a result of succession proceedings minus amortization due or pre-paid, and rescheduled debt service payments programmed to be paid out. Privatization proceeds for the central government include privatization proceeds and lump sum proceeds from concession fees in denar and foreign currency.

31. The general government fiscal balance includes, in addition to the central government fiscal balance, the financing position of the municipalities included in the definition of general government in $\$ 2$. It is expected that, quantitative targets for the general government fiscal balance will be set on the occasion of the $1^{\text {st }}$ semi-annual program review, 
provided that sufficiently comprehensive and reliable data on municipalities' finances will have become available.

32. The public sector fiscal balance includes, in addition to the general government fiscal balance, the financing position of the institutions included in the definition of public sector in $\$ 3$. Monitoring will also be done from the financing side.

\section{Adjustors}

33. The floors on central government fiscal balance will be adjusted upward by any shortfalls of gross external financing for central government's foreign financed projects spending (including the Road Fund) with respect to the following cumulative baseline:

End-September 2005

End-December 2005

End-March 2006

End-June 2006

End-September 2006

End-December 2006
EUR 26.7 million;

EUR 31.4 million;

EUR 43.0 million;

EUR 58.7 million;

EUR 65.2 million;

EUR 65.8 million.

\section{G. Central Government Wage Bill}

34. The ceiling on the central government wage bill includes central government wages and salaries, including allowances.

35. Annual ceilings have been established for the central government wage bill (Table 1 of the MEFP).

\section{H. Debt}

36. For assessing fiscal sustainability for program purposes, the standard analysis of the consolidated central government's debt sustainability will be complemented with an analysis that will include debt contracted and guaranteed by central and general governments and available information on government's contingent liabilities, such as external debt contracted by public and state owned enterprises without explicit government guarantees.

37. The limit on medium and long-term debt (Table 1 of the MEFP) applies to the contracting or guaranteeing by any branch of the government or the NBRM of new nonconcessional external debt with an original maturity of more than one year. This performance criterion applies not only to debt as defined in point No. 9 of the Guidelines on Performance Criteria with Respect to Foreign Debt adopted on August 24, 2000 by the 
Executive Board of the $\mathrm{IMF}^{1}$, but also to commitments contracted or guaranteed for which value has not been received. Excluded from this performance criterion are changes in indebtedness resulting from refinancing credits and rescheduling operations (including the deferral of interest on commercial debt), credits extended by the IMF and the BIS, and credits on concessional terms, defined as those with a grant element of 35 percent or more calculated using the OECD Commercial Interest Reference Rates (CIRRs) applicable for the program period. Specifically, the discount rates for debts with maturities less than 15 years will be based on the average CIRR of the previous 6 months, and for debts with maturities of 15 years and more the average CIRR of the previous 10 years. Debt falling within the limit shall be valued in euro at the exchange rate prevailing at the time the contract or guarantee becomes effective.

38. The limit on short-term debt (Table 1 of the MEFP) applies to the outstanding stock of short-term government and government-guaranteed external debt and the NBRM with an original maturity of up to and including one year. The term "debt" has the meaning set forth in point No. 9 of the Guidelines on Performance Criteria with Respect to Foreign Debt adopted on August 24, 2000 by the Executive Board of the IMF. Excluded from this performance criterion are changes in indebtedness resulting from rescheduling operations (including the deferral of interest on commercial debt), and normal import-related credits. Debt falling within the limit shall be valued in euro at the program exchange rates. There was no official short-term debt or guarantees on outstanding short-term debt as of endDecember 2004.

\section{External and Domestic Payments Arrears}

39. External payments arrears consist of the total past-due amounts of debt service obligations (interest and principal) on government, government-guaranteed, and the NBRM external debt, excluding arrears on external debt service obligations pending the conclusion of debt rescheduling agreements. ${ }^{2}$ Under the program, the nonaccumulation of external payments arrears is a continuous performance criterion. As of December 31, 2004 there were no outstanding external payment arrears as defined above.

40. Central government domestic arrears, excluding those to suppliers, are defined to include all payment delays to: (i) banks for bond payments (including for the repayment of frozen foreign currency deposits); (ii) individuals for Social Assistance Program payments;

\footnotetext{
${ }^{1}$ Decision No. 6230-(79/140) August 3, 1979, as amended by Decision Nos. 11096-(95/100), October 25, 1995, and 12274-(00/85), August 24, 2000. Under the Guidelines on Performance Criteria with Respect to Foreign Debt adopted on August 24, 2000 by the Executive Board of the IMF the definition of "debt" has been broadened with respect to the conventional definition to include, among other things, such instruments as financial leases.

${ }^{2}$ Amounts are only considered past-due after the contractual grace period expired.
} 
(iii) central government employees including for wages and salaries, and food and travel allowances; (iv) benefit recipients of the Child Care Program; (v) local governments. The definition excludes the customary lag in paying wages, social assistance and child allowance payments, and transfers to the extra-budgetary funds (in the following month after they accrue). According to the definition here, and as reported to IMF staff, central government domestic arrears, excluding those to suppliers, were denar 0 million as of endDecember 2004. Under the program, the outstanding stock of domestic arrears, as defined above, will not exceed at any time the amount outstanding as of end-December 2004.

41. Central government domestic arrears to suppliers are defined as obligations to suppliers which are due but not paid by more than 60 days and are non-disputed. As defined here, and as reported to Fund staff, the stock of arrears to suppliers stood at denar 2,019 million as of end-December 2004. Under the program, the outstanding stock of domestic arrears, as defined above, will not exceed the amount outstanding as of endDecember 2004.

42. A separate sub-ceiling is set for the Health Fund arrears, as defined above in $\uparrow 41$ and as reported to Fund staff. Under the program the aggregate outstanding stock of arrears will not exceed the amount outstanding as of end-2004. (The aggregate stock of these arrears stood at denar 1,710 million as of end-December 2004.)

\section{J. Valuation}

\section{Valuation of the NBRM balance sheet and the monetary survey}

43. For the programmed foreign exchange projections, the program exchange rates were applied.

44. For program purposes, all foreign currency-related assets and liabilities will be evaluated at program exchange rates. For 2005 and 2006, the program exchange rates are those that prevailed on December 31, 2004. In particular, EUR1 $=61.3100$ denars, US $\$ 1=$ 45.0676 denars; SDR $1=69.9903$ denars, and EUR1=1.3604 U.S. dollars.

45. Performance will be measured in constant exchange rates. For this methodology, assets and liabilities of the banking system will be valued as follows: The stocks of assets and liabilities denominated in foreign currencies outstanding at December 31, 2004 are valued at the program exchange rates defined above in $\$ 44$. Gold is valued at the price fixed in the London market at end-December 2004 (US\$ 438.00 per ounce). Changes in assets and liabilities will also be valued at these exchange rates. The exchange rate effects on the foreign currency denominated assets and liabilities of commercial banks will be estimated on the basis of their currency composition, as provided by the NBRM banking supervision department. 


\section{Valuation of NIR}

46. For the programmed foreign exchange projections, the program exchange rates were applied.

47. For program monitoring, the NBRM estimates the valuation effects on the NIR of the NBRM as follows. On a daily basis all foreign currency denominated balances are converted into Euros using the middle rates from the NBRM official exchange rate list for the same day. These balances are compared to the balances in Euros at the end of the previous day calculated in the same way (i.e., using the middle rates from the NBRM official exchange rate list for that day). The change in the daily Euro denominated balances, so calculated, is compared to the recorded daily transaction flows converted in Euros using the same methodology. Any difference between the two values is attributed to valuation effects.

\section{Valuation of the fiscal deficit}

48. For the programmed foreign exchange projections, the program exchange rates were applied.

49. For fiscal deficit measuring purposes, the foreign currency component of deficit financing will also be converted into denars at constant program exchange rates.

\section{K. Monitoring and Reporting Requirements}

50. Performance under the program will be monitored from information provided to the IMF by the NBRM and the Ministry of Finance. All data will be monthly, unless otherwise specified, and should be submitted by the authorities to the IMF staff within 30 days of the end of each month, for the minimum of three consecutive months prior to the IMF Board discussion on the arrangement, unless otherwise specified. In addition, data on performance at the program test dates will be submitted with a cover letter signed by an authorized official.

51. The following information will be supplied to the IMF by the Ministry of Finance: (i) fiscal table for the consolidated central government (i.e., including special revenue accounts and the extra-budgetary funds) (40 days after the end of each month); (ii) monthly information on privatization receipts (including detailed description of cash payments in local and foreign currency and payments with government bonds); (ii) data on workers registered as unemployed with the unemployment fund; (iv) information on guarantees given on new debt, on new debt contracted by the government, government agencies, and public enterprises and on debt stock in gross and net terms; (v) information on domestic arrears, including to suppliers and distinguishing between court disputed and non-disputed arrears; (vi) data on outlays on structural reforms and public administration reforms; (vii) data on the claimed and unclaimed portion of the repayment of frozen foreign currency deposits; and (ix) information on the local government fiscal accounts (starting August 2005). In addition, the MOF, in coordination with the NBRM, will provide quarterly reports on the reconciliation of 
monetary and fiscal data on net government position vis-à-vis the banking system, and data on the holders of government securities broken down between banks and non-bank entities.

52. The NBRM will supply: (i) balance sheets of the NBRM and the consolidated accounts of the commercial banks - both should include details of the credit and deposits position of funds and other government entities as listed in $₫ 1$ (20 days after the end of the month); (ii) the monetary survey; (iii) data on components of NIR of the NBRM as defined in section B, valued in euros adjusted for valuation changes; (iv) statement from the Road Fund indicating its balances (in denar and foreign currency) at the NBRM and at the commercial banks separately; (v) the foreign exchange cash flow of the NBRM, including the level of official reserves, if necessary, reconciled with the NBRM balance sheet data for NFA; (vi) daily and monthly closing and average exchange rates; (vii) detailed data on exports and imports; (viii) information on all overdue payments on short-term external debt and on medium- and long-term external debt; (ix) data on foreign borrowing including gross disbursements, amortization, and interest payments by debtors (central government, agencies and public enterprises); (x) information on lending by domestic money banks according to credit ratings of borrowers; (xi) data on off-balance sheet activity of domestic money banks; and (xii) data on each domestic money banks' compliance with prudential regulations will be provided on a quarterly basis within 30 days after the end of the quarter; (xiv) Detailed reporting on commercial banks assets and liabilities, including breakdowns by currency, currency indexation, maturity and sector, on a quarterly basis; (xv) Quarterly information on commercial banks lending and deposits indexed to foreign currencies; (xvi) information on deposit and lending interest rates monthly. Monthly data on all components of balance of payments will be submitted within $2 \frac{1}{2}$ months of the end of each month. By the end of the following months, the NBRM will provide preliminary trade data based on customs information. Data on stock of external debt will be provided on a quarterly basis, within 30 days of the end of the quarter.

53. The State Statistics Office (SSO) office will supply monthly updates on CPI, PPI, industrial production, wages, employment, and import and export data. The SSO will also prepare quarterly GDP data three months after the end of the quarter. In case of changes in the statistical methodology, the SSO will provide data in the new and old methodology for at least one reporting point. The SSO will also provide updates when historic time series get corrected. All submissions will be in electronic form. 
Table 1. Net International Reserves of the NBRM

(In millions of euros) 1/

End-Dec. 2004

Net international reserves [1 - 2]

613.0

1. Reserve assets [1.1 - 1.2]

717.0

1.1. Foreign assets

728.9

Gold

63.6

Demand deposits in foreign banks

183.2

Time deposits in foreign banks

460.6

Foreign currency at the NBM

Foreign securities

18.2

Letters of credits

0.0

Guarantees

0.0

Checks

0.0

1.2. Foreign assets excluded from reserve assets $\quad 11.9$

$\begin{array}{ll}\text { Subordinated loan (AY Bank) } & 3.7\end{array}$

Booked funds (formerly AY Bank) $\quad 7.6$

$\begin{array}{ll}\text { Other pledged assets } & 0.0\end{array}$

Other FA excluded from gross reserves $\quad 0.6$

2. Reserve liabilities [2.1 +2.2] 104.0

$\begin{array}{ll}\text { 2.1. To nonresidents } & 46.7\end{array}$

$\begin{array}{ll}\text { IMF } & 46.0\end{array}$

BIS $\quad 0.0$

Other 0.7

2.2. To residents $2 / \quad 57.3$

Foreign currency deposits of banks $\quad 2.4$

Required reserves $\quad 54.9$

$\begin{array}{ll}\text { Other } & 0.0\end{array}$

Memorandum items:

Net foreign assets [1.1 - 2.1] $\quad 682.2$

Foreign currency deposits of central government 2/ $\quad 113.6$

Valuation changes to NIR (cumulative from end-2002

NIR excluding valuation changes

613.0

Source: NBRM

1/ Valued at program exchange rates (denar/U.S. dollar: 45.0676, denar/euro: 61.3100;

U.S. dollar/euro;1.3604).

2/ Foreign currency deposits of central government are excluded from reserve liabilities. 
Table 2. Balance of Payments Financing Assumptions, from end-December 2004 to end December 2006 1/

(quarterly flows in millions of euros, unless otherwise indicated)

\begin{tabular}{|c|c|c|c|c|c|c|c|c|}
\hline & Mar-05 & Jun-05 & Sep-05 & Dec-05 & Mar-06 & Jun-06 & Sep-06 & Dec-06 \\
\hline Grants & 0.0 & 6.3 & 0.0 & 7.0 & 0.0 & 0.0 & 0.0 & 0.0 \\
\hline The Netherlands & 0.0 & 6.3 & 0.0 & 7.0 & 0.0 & 0.0 & 0.0 & 0.0 \\
\hline Loans & 0.0 & 19.2 & 6.8 & 185.6 & 11.2 & 0.0 & 0.0 & 22.1 \\
\hline IBRD/IDA & 0.0 & 14.7 & 0.0 & 22.1 & 0.0 & 0.0 & 0.0 & 22.1 \\
\hline Euro Bond & 0.0 & 0.0 & 0.0 & 150.0 & 0.0 & 0.0 & 0.0 & 0.0 \\
\hline EBRD (ESM pre-privatization loan proceeds) & 0.0 & 4.5 & 6.8 & 13.5 & 11.2 & 0.0 & 0.0 & 0.0 \\
\hline Privatization proceeds in foreign currency & 0.0 & 0.0 & 0.0 & 0.0 & 250.0 & 0.0 & 0.0 & 0.0 \\
\hline Lump-sum proceeds from concession fees & 0.0 & 0.0 & 0.0 & 0.0 & 0.0 & 0.0 & 0.0 & 0.0 \\
\hline Restitution of foreign assets of the former SFRY & 0.0 & 0.0 & 0.0 & 6.5 & 0.0 & 0.0 & 0.0 & 0.0 \\
\hline Prepayment of foreign loans (minus) & 0.0 & 0.0 & 0.0 & 0.0 & -120.1 & 0.0 & 0.0 & 0.0 \\
\hline \multicolumn{9}{|l|}{ Total } \\
\hline BOP financing assumptions for TMU (quarterly flows) & 0.0 & 25.5 & 6.8 & 199.0 & 141.2 & 0.0 & 0.0 & 22.1 \\
\hline BOP financing assumptions for TMU (cumulative from end-December 2002) & 0.0 & 25.5 & 32.3 & 231.3 & 372.5 & 372.5 & 372.5 & 394.5 \\
\hline
\end{tabular}

1/ At program exchange rates. 


\section{Debt Sustainability Analysis}

\section{A. Fiscal Sustainability}

\section{Under baseline assumptions, the central government gross debt-to-GDP ratio} declines substantially over the medium term. The baseline scenario assumes average GDP growth of 4.3 percent, an inflation rate of 1.9 percent and a primary fiscal surplus of 0.6 percent. In this scenario, the debt ratio rises sharply in 2005, owing to a planned Eurobond issue and snaps back in 2006 when the proceeds of that issue are used to retire external debt (Table 1). Thereafter it declines steadily, reaching 28 percent in 2010 . The decline is explained mainly by a tight fiscal stance and favorably automatic debt dynamics, notably the robust growth of real GDP. The authorities' intend to deposit the proceeds from large privatizations at the NBRM in order to support a significant increase in foreign exchange reserves. These deposits will provide an additional financial cushion which is not taken into account in the debt sustainability analysis owing to its focus on gross debt.

\section{Available data on broader measures of public debt also point to declining debt}

ratios. For the time being, the lack of reliable and comprehensive financial data on municipalities and public enterprises precludes a full-fledged debt sustainability analysis of the consolidated general government or the public sector. ${ }^{1}$ However, municipal debt (mostly arrears) is only about one percent of GDP, which suggests that broadening the coverage to general government would not fundamentally alter the debt sustainability outlook, assuming that municipalities maintain prudent fiscal policies during the projection period. ${ }^{2}$ Similarly the external debt ratio of public enterprises as of end-2005 amounts to about 3.3 percent of GDP (mostly covered by government guarantees) and is projected to remain broadly stable. Consequently, broadening the analysis to cover public enterprises is unlikely to alter the conclusions drawn from the analysis of the central government debt.

\section{Fiscal debt dynamics remain sustainable also under the alternative scenarios.} When key variables are set at their historical average values (Scenario A1), the central government gross debt-to-GDP ratio declines during the projection period (albeit less than in

\footnotetext{
${ }^{1}$ More comprehensive and reliable data on municipalities' finances will become available during 2006.

${ }^{2}$ Until mid-2007, local governments are constrained in their borrowing by a provision in the Law on Local Government Financing that requires central government approval for local government borrowing. After that, the framework for local government borrowing will need to be reconsidered taking into account their track record in clearing arrears. In addition, limits should be established for local government debt.
} 
the baseline) reaching 35 percent by $2010 .^{3}$ The no-policy-change scenario (A2) yields results that are similar to the baseline. A country-specific scenario (A3) shows how a twoyear shock to real growth (real GDP growth in 2006 and 2007 is at historical average minus one standard deviation) increases the end-2007 debt ratio by slightly over 4 percent relative to baseline. ${ }^{4}$ However, as the growth reverts to baseline, the debt ratio declines during the remainder of the projection period.

\section{The standard bound tests suggest that sustainability is robust under all the} shocks except the GDP shock which, in this case, is implausibly large. Isolated shocks on the interest rate (B1), the primary balance (B3), the realization of larger than expected contingent liabilities (B6), and a combination of shocks on real growth, interest rate and primary balance (B4) would all result in moderate one-time increases in the debt ratio. However, these effects decay so that, by the end of the projection period, the debt ratio in all scenarios is below or close to the end-2005 level. A one-off exchange rate shock (B5) increases the debt ratio by a larger amount, but in this case too, the ratio soon returns to a declining path. The standard shock to real growth (B2: GDP growth at historical average minus two standard deviations), undermines debt sustainability. However, owing to Macedonia's low and volatile growth during the period that is used in calibrating this shock, i.e., the period that covers both the 1999 Kosovo crisis and the 2001 security crisis, this shock implies a highly unlikely 10 percent cumulative decline in GDP over two years.

\section{While the preceding analysis suggests that FYR Macedonia is in a relatively} comfortable fiscal position, there is no room for complacency. While the projections assume that the fiscal stance is kept tight throughout the projection horizon there are risks of fiscal slippage. Also, the maturity profile of domestic debt is becoming less favorable as the authorities amortize long-term bonds by issuing securities with much shorter maturities. This increases gross financing needs and underscores the need for a debt strategy that pays due regard to the liquidity risks.

\section{B. External Sustainability}

\section{In the baseline scenario, FYR Macedonia's gross external debt ratio rises in} 2005 and subsequently declines throughout the projection period. ${ }^{5}$ In 2005 , the debt ratio increases by some 2 percentage points, reflecting primarily the build up of gross reserves

\footnotetext{
${ }^{3}$ Owing to the lack of reliable and comprehensive debt data for the period prior to 1999 , only 6 years of data was used in calculating historical averages and in calibrating standard alternative shocks and bound tests.

${ }^{4}$ This scenario assumes that the revenue-to-GDP ratio is identical with that of the baseline.

${ }^{5}$ The projections include an estimate of trade credits. Consequently the debt stock is higher than in earlier DSA tables for FYR Macedonia, which excluded trade credit.
} 
through higher government deposits at the NBRM, financed mainly through a planned Eurobond issuance (Table 2). In 2006, the debt ratio declines by close to 2 percentage points as the bulk of the proceeds from the Eurobond are used for retiring external debt. Thereafter, the debt ratio declines gradually as net FDI inflows and automatic debt dynamics (in particular the robust real GDP growth) more than offset the still sizable current account deficits. By 2010, the debt ratio reaches 43 percent of GDP. Because part of the external financing inflows - most notably privatization receipts - is used to build up gross reserve coverage, the decline in net external debt (i.e., gross debt minus gross foreign reserves) would be more pronounced than for the gross debt figures discussed above. Consequently, the very modest decline in the external gross debt ratio during the projection period masks part of the underlying improvement in the external debt position. Another notable feature during the projection period is the increasing share of trade credits in the total external debt. While these credits are normally rolled over and are therefore "self-financing", their increase does underscore the need to increase gross reserves in parallel with growing imports to ensure that reserves remain sufficient to accommodate disruptions in normal import financing practices (i.e., the risk and trade credit lines would suddenly be cut during exceptional circumstances).

\section{Under the standard alternative scenario (A1), which is based on key variables} reverting to historical levels, the external debt dynamic could become unsustainable. The debt-to-GDP ratio increases steadily, reaching 68 percent in 2010 (Table 2). Of course, given FYR Macedonia's history of slow growth and wide current account deficits, this scenario represents a particularly weak performance. Also, the level of external debt at the end of the projection horizon, while some 25 percentage points higher than in the baseline, would remain manageable if the economic performance of the country were to improve after 2010. In an alternative country specific scenario (A2), in which real GDP growth in 2006 and 2007 is at 6-year historical average minus one standard deviation, the debt ratio increases by about 5.5 percentage relative to the baseline by 2007 and declines gradually thereafter as growth returns to the baseline path.

\section{The bound tests also point to some vulnerabilities although in all cases the debt} ratio would return to a declining path. Temporary two-year shocks to interest rate (B1) and real GDP growth (B2) result in higher debt ratios in 2007 but the impact decays thereafter and by 2010 the debt ratios would be close to the 2005 levels. A one-time 30 percent nominal depreciation (B6) and a two-year shock to the non-interest current account (B4) would result in similar dynamics, though with debt ratios remaining somewhat more above the starting level. The other two bound tests (B3 and B5) result in significantly higher initial increases in debt ratios but in all cases the ratios return to declining paths immediately after the shocks are over. Bound test B5, which represents a combination of smaller shocks, gives rise to the largest increase in the debt ratio. In this scenario, the end-2010 debt ratio is 72 percent, 29 percentage points higher than in the baseline scenario.

9. The external debt sustainability analysis underscores the need for a major reform program, as is being supported under the proposed SBA. It is striking that the 
scenario based on historical averages results in the most worrying debt dynamics. This highlights the disappointing economic performance during the past decade and underscores the need to reduce the current account deficit and implement structural reforms that will improve business climate and help in attracting sufficient FDI inflows. Indeed, the clear message is that "business as usual" would not ensure external sustainability going forward. 


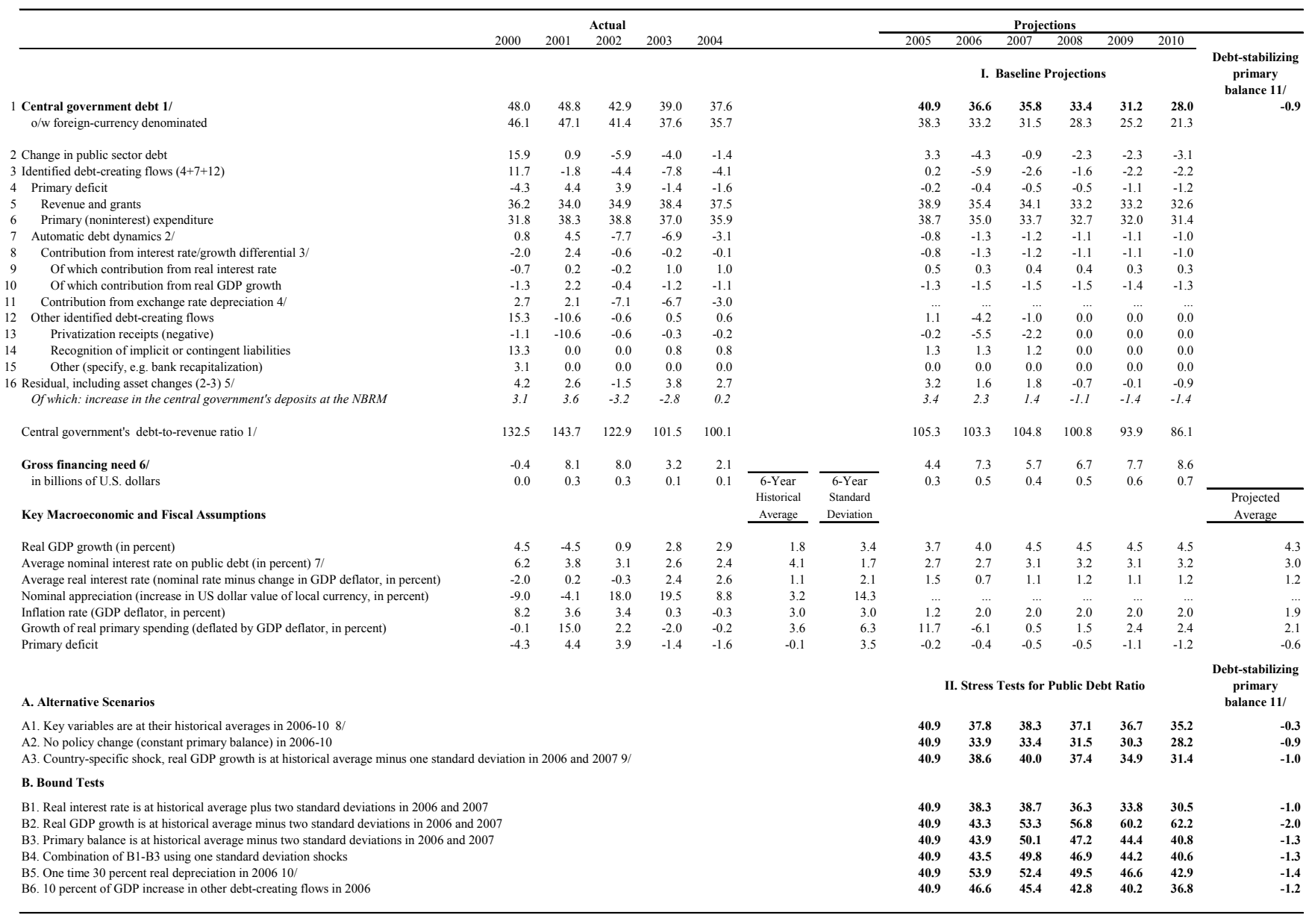

1/Consolidated central government gross debt.

2/ Derived as $[(\mathrm{r}-\pi(1+\mathrm{g})-\mathrm{g}+\alpha \varepsilon(1+\mathrm{r})](1+\mathrm{g}+\pi+\mathrm{g} \pi))$ times previous period debt ratio, with $\mathrm{r}=$ interest rate; $\pi=$ growth rate of GDP deflator; $\mathrm{g}=$ real GDP growth rate; $\alpha=$ share of foreign-currency

denominated debt; and $\varepsilon=$ nominal exchange rate depreciation (measured by increase in local currency value of U.S. dollar).

$3 /$ The real interest rate contribution is derived from the denominator in footnote $2 /$ as $r-\pi(1+\mathrm{g})$ and the real growth contribution as $-\mathrm{g}$.

Tefined as consolidat central government deficit, plus

7/ Derived as nominal interest expenditure divided by previous period debt stock.

$8 /$ The key variables include real GDP growth; real interest rate; and primary balance in percent of GDP.

9/ For more detalis about this scenario, see the text.

(based on GDP deflator) -

11 / Assumes that key variables (real GDP growth, real interest rate, and other identified debt-creating flows) remain at the level of the last projection year. 


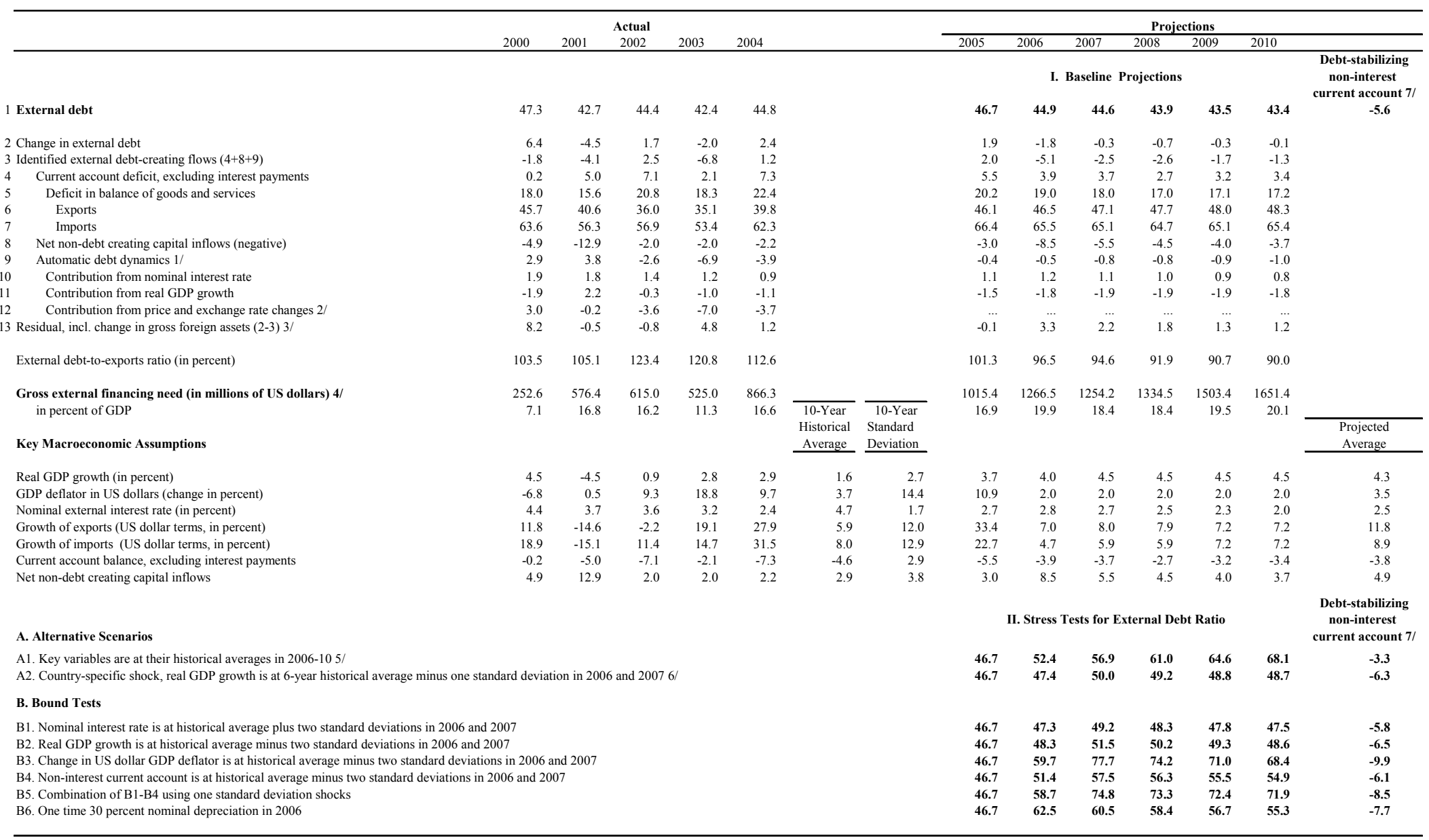

$1 /$ Derived as $[\mathrm{r}-\mathrm{g}-\rho(1+\mathrm{g})+\varepsilon \alpha(1+\mathrm{r})](1+\mathrm{g}+\rho+\mathrm{g} \rho)$ times previous period debt stock, with $\mathrm{r}=$ nominal effective interest rate on external debt; $\rho=$ change in domestic GDP deflator in US dollar terms, $\mathrm{g}=$ real GDP growth rate,

$\varepsilon=$ nominal appreciation (ncrease in dollar value of domestic currency), and $\alpha=$ share of domestic-currency denominated debt in total external debt.

$2 /$ The contribution from price and exchange rate changes is defined as $[-\rho(1+\mathrm{g})+\varepsilon \alpha(1+\mathrm{r})] /(1+\mathrm{g}+\rho+\mathrm{g} \rho)$ times previous period debt stock. $\rho$ increases with an appreciating domestic currency $(\varepsilon>0)$ and rising inflation $($ based on GDP deflator).

3/ For projection, line includes the impact of price and exchange rate changes.

4/ Defined as current account deficit, plus amortization on medium- and long-term debt, plus short-term debt at end of previous period.

deflator growth; and both non-interest current account and non-debt inflows in percent of GDP.

$7 /$ ong-rin, contant blance that stailizes the debt

of the last projection year. 
FYR Macedonia-Financial Position in the Fund

(as of June 30, 2005)

I. Membership Status:

II. General Resources Account:

Quota

Fund holdings of currency

III. SDR Department:

Net cumulative allocation

Holdings

IV. Outstanding Purchases and

Loans:

Extended Arrangements

PRGF Arrangements

Stand-By Arrangements

Systemic Transformation Facility
Joined 12/14/92; Article VIII

SDR Million
68.90
89.86

$\underline{\text { SDR Million }}$

8.38

1.16

$\underline{\text { SDR Million }}$

0.96

15.36

20.00

22.30

2.07

29.03

3.00

V. Latest Financial Arrangements:

Approval Expiration

Type

Stand-By

Date

EFF

04/30/2003

Date

PRGF

$11 / 29 / 2000$

$08 / 15 / 2004$

$11 / 22 / 2001$

$12 / 18 / 2000$

$11 / 22 / 2001$
Amount Approved

(SDR Million)

20.00

24.12

10.34
Amount Drawn

(SDR Million)

20.00

1.15

1.72

VI. Projected Payments to the Fund (Expectations Basis) ${ }^{1}$

(SDR million; based on existing use of resources and present holdings of SDRs):

\begin{tabular}{lrrrrr} 
& \multicolumn{5}{c}{ Forthcoming } \\
\cline { 2 - 6 } & 2005 & 2006 & 2007 & 2008 & 2009 \\
Principal & 3.92 & 13.18 & 14.27 & 4.25 & 0.34 \\
Charges/Interest & $\underline{0.52}$ & $\underline{0.90}$ & $\underline{0.54}$ & $\underline{0.25}$ & $\underline{0.19}$ \\
Total & $\underline{\underline{4.44}}$ & $\underline{\underline{4.08}}$ & $\underline{\underline{14.82}}$ & $\underline{4.51}$ & $\underline{0.53}$
\end{tabular}

\footnotetext{
1 This schedule presents all currently scheduled payments to the IMF, including repayment expectations and repayment obligations. The IMF Executive Board can extend repayment expectations (within predetermined limits) upon request by the debtor country if its external payments position is not strong enough to meet the expectations without undue hardship or risk, (see repayment schedules and IMF lending for details).
} 


\section{Projected Payments to the Fund (Obligations Basis) ${ }^{2}$}

(SDR million; based on existing use of resources and present holdings of SDRs):

Forthcoming

\begin{tabular}{lrrrrr}
\hline 2005 & 2006 & 2007 & 2008 & 2009 \\
& & & & \\
2.82 & 6.99 & 12.08 & 10.35 & 3.54 \\
$\underline{0.52}$ & 1.01 & $\underline{0.87}$ & $\underline{0.53}$ & $\underline{0.26}$ \\
$\underline{\underline{3.35}}$ & $\underline{8.00}$ & $\underline{12.95}$ & $\underline{10.88}$ & $\underline{3.80}$
\end{tabular}

VII. Safeguards Assessments:

Under the Fund's safeguards assessments policy, the National Bank of the Republic of Macedonia is subject to an updated safeguards assessment with respect to the proposed Stand-By Arrangement. An earlier safeguards assessment was completed on April 24, 2003, and proposed measures to address weaknesses, and their implementation continues to be monitored by staff under the present Stand-By Arrangement.

\section{Exchange Arrangement:}

The currency of the FYR Macedonia is the denar. The FYR Macedonia maintains a managed floating exchange rate system with a de facto peg to the Euro.

Households can transact only through commercial banks or through foreign exchange bureaus that act as agents of banks; enterprises can transact through the banking system. The reserve requirement on all foreign currency deposits is set at 10 percent.

At end-June 2005, the official exchange rate was denar 50.86 per U.S. dollar and denar 61.30 per euro. The FYR Macedonia has accepted the obligations of Article VIII, Sections 2, 3, and 4 with effect from June 19, 1998. The FYR Macedonia maintains an exchange restriction subject to the Fund's approval under Article VIII, Section 2(a) arising from restrictions imposed on the transferability of proceeds from current international transactions contained in former frozen foreign currency saving deposits. The retention of this restriction was approved by the

\footnotetext{
2 This schedule is not the currently applicable schedule of payments to the IMF. Rather, the schedule presents all payments to the IMF under the illustrative assumption that repayment expectations-except for SRF repayment expectations-would be extended to their respective obligation dates by the IMF Executive Board upon request of the debtor country (see repayment schedules and IMF lending for details). SRF repayments are shown on their current expectation dates, unless already converted to an obligation date by the IMF Executive Board.
} 
Board on June 27, 2005 until April 30, 2006 or the completion of the next Article IV consultation, whichever is earlier.

\section{Article IV Consultations:}

The first consultation with the FYR Macedonia was concluded in August 1993. The last consultation was concluded on April 30, 2003. The FYR Macedonia is on the standard consultation cycle.

X. Technical Assistance (since 1999):

Purpose

BOP Statistics

Tax administration

Reserve management

Monetary Statistics

Fiscal ROSC

Debt management

National Accounts

Debt Management

Data ROSC

Fiscal Decentralization

FSAP

Public Expenditure Management

Monetary Operations and Payment System

Balance of Payments Statistics

Tax Policy

Development of a Treasury System
Department Date

STA

FAD

MFD

STA

FAD

FAD

STA

FAD

STA

FAD

MFD/WB

FAD

MFD

July 2005

May 2005

May 2005

April 2005

February 2004

October 2004

May 2004, October 2004

March 2004

February 2004

September 2003

March 2002

May 2003 and June 2003

May 2003

November-December 2002

April 2004

STA

June-August, 2000

October 2002

FAD

December 2001 
November 2001

Value-Added Tax

FAD

February 1999

October 1999

October 2000

Article VIII

Monetary Policy and Banking Supervision

MFD

MFD

Value-Added Tax

FAD

FAD
October 2004-April 2005

September 2000

October 1999

December 1999, May 2000

October 1999-April 2001

January 2000-July 2001

\section{Resident Representative}

The Fund has had a resident representative in Skopje since 1995. Mr. Kevin Ross has held this position since May 2003. 


\section{FYR Macedonia: Statistical Issues}

The authorities, with technical assistance from the Fund and other bilateral and multilateral agencies, have made significant progress in upgrading the country's statistical system in recent years. Continued efforts are being made to further improve data quality and availability. The authorities mostly report data to the Fund on a timely basis and an IFS page is available. The authorities began participating in the General Data Dissemination System (GDDS) in February 2004. A draft data ROSC was prepared in February 2004, and the report was posted on the IMF's website in September 2004.

Poor real sector data still hamper economic analysis despite some improvements. Quarterly GDP volume estimates are produced on a regular basis and their quality benefited from improvements in the compilation of industrial production indicators which are used as a basis for the quarterly GDP estimates. In addition, the State Statistics Office (SSO) is currently improving the quarterly GDP compilation (using the expenditure and the production approach) with support from STA. However, the implementation of STA's recommendations has been slow. Severe shortcomings also remain in the preparation of deflators used for GDP at constant prices. A STA mission to provide technical assistance on national accounts, taking place during July 25-August 5, 2005, will further review progress. The SSO has recently improved consumer price compilation by a revision of weights in the consumer basket. Employment data from a company survey continues to be unreliable, but the timeliness of unemployment and labor force data has been improved as the labor force survey is now conducted on a quarterly basis (compared to annual surveys in the past). Wage data suffers from the volatility of wage arrears payments.

The compilation and coverage of balance of payments data have improved in recent years. The October 2002 STA technical assistance mission recommended further improvements to the estimation of short-term trade credits and investigated the large errors and omissions. Recommendations were made relating to the valuation of imports of goods and to the estimation of transportation services. Most of the recommendations were implemented by the authorities, but gaps remain because data from surveys and reports on transportation services, direct investment, and transactions settled through resident accounts with non-resident banks were not incorporated into the statistics. The 2004 data ROSC mission confirmed these shortcomings. The February 2005 STA technical assistance mission focused on the validation and inclusion of surveys and reports in the compilation of balance of payments and international investment position.

Since the 2004 staff report, progress has been made in government finance statistics. Data for the consolidated central government are now available following the expansion of coverage to extrabudgetary funds and special revenue accounts (SRAs), including their foreign-financed projects. The financing data for the central government is presented increasingly in line of the GFSM 1986. The main remaining deficiency is the lack of data on on-lending. The authorities have agreed to prepare by June 2006 a report on on-lending and, based on findings of this report, to take steps to incorporate on-lending into the budget. As 
part of the ongoing process of fiscal decentralization, the authorities are committed to start collecting data on municipalities that will allow monitoring of general government's finances in 2006. Against this background, implementation of the GFSM 2001 in line with the recommendations of the 2004 data ROSC mission has been given a lower priority-although introducing a full set of integrated accounts, including stocks and flows, will greatly enhance the consistency and comprehensiveness of the data. The financing data for the central government from the BOP and the monetary survey are now being reconciled on a regular basis in line with staff's recommendations and discrepancies have been significantly reduced. The data for extra-budgetary funds and SRAs remain less reliable than other central government data. Data on central government domestic arrears has improved following introduction of commitment accounting in autumn 2003. Data on funds' arrears, especially of the Health Insurance Fund, remain unreliable. In 2005, the authorities started systematic compilation of public debt data and are planning to start publishing statistics on public debt on a regular basis during the course of 2006. Government Finance Statistics are not reported to the Fund for inclusion in the Government Finance Statistics Yearbook or the IFS.

Money and banking data are reported to the Fund on a regular basis, although in the past, they have been subject to concerns regarding inconsistencies, accuracy and reliability. To address these issues, STA provided technical assistance in April 2005 based on the findings of a data ROSC in 2004. The mission and the authorities agreed on an action plan aimed at improving current data collection and compilation practices. Specific recommended actions include: (i) improved institutional coverage of monetary statistics through the inclusion of savings houses in the reporting of other depository corporations; (ii) the collection of more disaggregated data in both the National Bank of Republic of Macedonia (NBRM) and commercial banks' balance sheets for the improved classification of accounts by economic sector; (iii) improved data consistency through reconciliation of loan data among various sectors; (iv) the publication of improved interest rate data in IFS; and (v) the establishment of a well-defined policy on data revisions in line with best international practice. The mission was of the view that the NBRM can implement many of these recommendations, although additional technical assistance might be necessary once further financial sector development takes place. 
FYR MACEDONIA: TABLE OF COMMON INDICATORS REQUIRED FOR SURVEILLANCE

(as July 29, 2005)

\begin{tabular}{|c|c|c|c|c|c|c|c|}
\hline & Date of & Date & Frequency & Frequency & Frequency & Mem & Items: \\
\hline & $\begin{array}{c}\text { latest } \\
\text { observation }\end{array}$ & received & Data $^{6}$ & $\begin{array}{c}\text { of } \\
\text { Reporting }\end{array}$ & $\begin{array}{c}\text { of } \\
\text { publication }\end{array}$ & $\begin{array}{c}\text { Data Quality - } \\
\text { Methodological } \\
\text { soundness }^{7} \\
\end{array}$ & $\begin{array}{c}\text { Data Quality - } \\
\text { Accuracy and } \\
\text { reliability } \\
\end{array}$ \\
\hline Exchange Rates & $6 / 30 / 05$ & $7 / 15 / 05$ & $\mathrm{D}$ and $\mathrm{M}$ & $\mathrm{W}$ and $\mathrm{M}$ & $\mathrm{D}$ and $\mathrm{M}$ & & \\
\hline $\begin{array}{l}\text { International Reserve Assets and Reserve Liabilities of the Monetary } \\
\text { Authorities } 1\end{array}$ & $7 / 15 / 05$ & $7 / 22 / 05$ & M & M & M & & \\
\hline Reserve/Base Money & $6 / 30 / 05$ & $7 / 25 / 05$ & $\mathrm{D}$ and $\mathrm{M}$ & $\mathrm{W}$ and $\mathrm{M}$ & M & & \\
\hline Broad Money & $6 / 30 / 05$ & $7 / 25 / 05$ & M & M & M & $\mathrm{O}, \mathrm{LO}, \mathrm{LO}, \mathrm{O}$ & $\mathrm{O}, \mathrm{LO}, \mathrm{O}, \mathrm{O}, \mathrm{O}$ \\
\hline Central Bank Balance Sheet & $6 / 30 / 05$ & $7 / 25 / 05$ & M & M & M & & \\
\hline Consolidated Balance Sheet of the Banking System & $6 / 30 / 05$ & $7 / 22 / 05$ & M & M & M & & \\
\hline Interest Rates ${ }^{2}$ & $6 / 30 / 05$ & $7 / 04 / 05$ & M & M & M & & \\
\hline Consumer Price Index & Jun 2005 & $7 / 15 / 05$ & M & M & M & $\mathrm{O}, \mathrm{O}, \mathrm{O}, \mathrm{LO}$ & $\mathrm{LO}, \mathrm{O}, \mathrm{LNO}, \mathrm{O}, \mathrm{LO}$ \\
\hline $\begin{array}{l}\text { Revenue, Expenditure, Balance and Composition of Financing }{ }^{3}-\text { General } \\
\text { Government }\end{array}$ & NA & NA & NA & NA & NA & & \\
\hline $\begin{array}{l}\text { Revenue, Expenditure, Balance and Composition of Financing }{ }^{3}-\text { Central } \\
\text { Government }\end{array}$ & $5 / 31 / 05$ & $7 / 1 / 05$ & M & M & M & LO, LNO, LO, O & $\begin{array}{l}\text { LO, LO, LO, LO, } \\
\text { LNO }\end{array}$ \\
\hline $\begin{array}{l}\text { Stocks of Central Government and Central Government-Guaranteed } \\
\text { Debt }^{5}\end{array}$ & $5 / 31 / 05$ & $7 / 1 / 05$ & M & M & I & & \\
\hline External Current Account Balance & Mar 2005 & $6 / 15 / 05$ & M & I & Q & & \\
\hline Exports and Imports of Goods and Services & Mar 2005 & $6 / 15 / 05$ & M & I & Q & $\mathrm{O}, \mathrm{LO}, \mathrm{O}, \mathrm{LO}$ & LO, O, LO, O, LO \\
\hline GDP/GNP & Mar 2005 & $7 / 15 / 05$ & Q & Q & Q & $\mathrm{O}, \mathrm{LO}, \mathrm{O}, \mathrm{LO}$ & $\mathrm{LO}, \mathrm{O}, \mathrm{LNO}, \mathrm{O}, \mathrm{O}$ \\
\hline Gross External Debt & $5 / 31 / 05$ & $7 / 01 / 05$ & M & M & M & & \\
\hline
\end{tabular}

${ }^{1}$ Includes reserve assets pledged or otherwise encumbered as well as net derivative positions.

${ }^{2}$ Both market-based and officially-determined, including discount rates, money market rates, rates on treasury bills, notes and bonds

${ }^{3}$ Foreign, domestic bank, and domestic nonbank financing.

${ }^{4}$ The general government consists of the central government (budgetary funds, extra budgetary funds, and social security funds) and state and local governments.

${ }^{5}$ Currency and maturity composition is reported only on request.

${ }^{6}$ Daily (D), Weekly (W), Monthly (M), Quarterly (Q), Annually (A), Irregular (I); Not Available (NA).

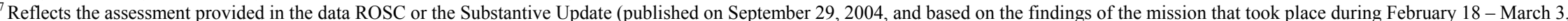

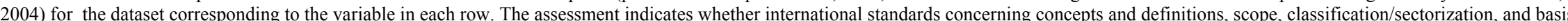

for recording are fully observed (O), largely observed (LO), largely not observed (LNO), or not observed (NO).

${ }^{8}$ Same as footnote 7 , except referring to international standards concerning source data, statistical techniques, assessment and validation of source data, assessment and validation of intermediate data and statistical outputs, and revision studies.

\author{
CInternational Monetary Fund. Not for Redistribution
}




\section{FYR Macedonia: IMF-World Bank Relations}

\section{A. Partnership in FYR Macedonia's Development Strategy}

1. FYR Macedonia has made considerable progress since independence in 1991, with support from the international community. While risks still remain, the relatively calm external and internal environment presents FYR Macedonia with perhaps the first significant window of opportunity since independence to focus on strengthening growth and employment opportunities. This follows the resolution of regional conflicts that took place through much of the 1990s, especially the cessation of hostilities in neighboring Kosovo, which particularly affected FYR Macedonia. Similarly, the Ohrid Framework Agreement of late 2001 has brought a reasonable measure of stability to the country, despite continuing isolated incidents. FYR Macedonia's progress is reflected perhaps most prominently in the agreement with the European Union (EU) on a Stabilization and Association Agreement, as well as FYR Macedonia's recent application to begin negotiations on EU membership.

2. Macro-economic stability has been largely maintained since the mid-1990s, and been further strengthened since 2002. Fiscal discipline and the peg to the Euro have helped to bring inflation close to zero, and resulted in Government spending falling as a proportion of GDP to a level significantly lower than most of the other former Yugoslav republics.

3. Nevertheless, macro-economic stability has not yet translated into sustained economic growth, and unemployment remains high and of serious concern. Growth has been largely positive since 1995, although with a contraction in 2001 associated with internal tensions. Yet the economy is still recovering from the dramatic collapse in output that took place through much of the late 1980s and early 1990s. While positive, growth also remains constrained by structural impediments. Further reform to improve competitiveness will be crucial to attract the investment needed to encourage stronger growth and to provide employment opportunities.

\section{B. World Bank Supported Reform}

4. Since FYR Macedonia joined the World Bank in 1993, 31 loans have been approved with a total value of approximately $\$ 680$ million. Just over half of all lending has been on concessional IDA terms. Reflecting improved economic performance and credit-worthiness, however, FYR Macedonia graduated fully from IDA in 2003. Although FYR Macedonia has received limited IBRD lending since it first started borrowing from the Bank in 1993, all new lending since 2003 has been on IBRD terms.

5. The World Bank's current program of assistance to FYR Macedonia is outlined in a Country Assistance Strategy discussed by the Board in September 2003. Reflecting Government priorities, the CAS outlines three broad areas of support: (i) promoting the efficient management of public resources and tackling corruption; (ii) promoting the creation of jobs through sustainable private sector led growth; and (iii) promoting social cohesion, 
building human capital, and protecting the most vulnerable. The CAS envisages a series of multi-sectoral development policy loans, supported by specific investment operations to build capacity to support priority reform areas. The CAS also provides for total financing in a high case lending scenario of up to $\$ 165$ million over three years. FYR Macedonia has qualified for such a high case lending scenario since the CAS was discussed by the Board.

6. World Bank operations in the past few years have focused primarily on supporting Macedonian Government efforts to strengthen public sector management and governance. A \$15 million Public Sector Management Adjustment Credit (PSMAC) approved in 2002, and a recently completed \$30 million Public Sector Management Adjustment Loan II (PSMAL II) have emphasized:

(i) improving governance of the health sector, particularly through reform of the Health Insurance Fund and international tendering for very significant pharmaceuticals contracts (which has resulted in savings to the Government of over $\$ 10$ million per annum);

(ii) strengthening overall budgetary management, especially though the consolidation of extra-budgetary funds within the Treasury Single Account; and

(iii) civil service reform, including the greater application of the merit principle in hiring and promotion decisions, and associated wage decompression to encourage retention of senior and experienced staff.

7. While maintaining involvement in key elements of the governance agenda, particularly health sector financing reform, the Bank's policy dialogue with the Government is increasingly shifting to structural economic reform to improve the business environment and promote growth and employment. Bank support for Macedonian Government reform efforts will be centered on a series of Programmatic Development Policy Loans (PDPLs), the first of which is intended to be presented to the Board in October 2005, shortly after IMF Board consideration of the proposed Stand-By Arrangement (SBA). In terms of strengthening the overall business and investment climate, the PDPLs will focus on supporting Government efforts to undertake judicial reform, labor market reform, and strengthened financial sector regulation and supervision. Efforts to further strengthen public sector governance will include an emphasis on further reform to improve the transparency of the critical health sector, building the capacity of the civil service to permit more effective strategic prioritization, and support for the decentralization program being conducted under the umbrella of the Ohrid Framework Agreement.

8. Development policy lending has been, and will continue to be, supported by a series of specific investments to build capacity to implement priority reforms. On the governance and public administration side, a Health Sector Management Improvement project will continue to provide technical assistance and investment support to the broader health sector reform program; a Social Protection project will continue to support reform in this area, 
including the proposed introduction of a second pillar pension system; and an Education project will continue to assist Government efforts to improve access and introduce stronger performance and equity measures in primary and secondary education financing. On the investment climate side, a Business Environment Reform and Institutional Strengthening (BERIS) project approved in June 2005 will concentrate on building capacity to improve business entry, operations, and exit; as well as enhancing the competitiveness of the enterprise sector; and a Real Estate Cadastre and Registration project approved in early 2005 is intended to strengthen land markets and the use of real property as collateral for business investment by providing more secure title. A Legal and Judicial Reform project currently under preparation is planned to be presented to the Board in early 2006 to support critical reforms in this area. Adjustment and investment lending has also been backed up by a comprehensive program of analytical work.

\section{IMF-World Bank Collaboration in Specific Areas}

9. World Bank and IMF engagement in FYR Macedonia in recent years has been marked by a spirit of collaboration and cooperation between the two institutions, and with the Government. Synergies between the proposed Bank and IMF programs over the next three years reflect a strong degree of consensus between both institutions and the Government in regard to reform priorities. Especially given the broad range of structural reform areas in which the Bank and the Fund will both be engaged, however, it will be critical that close coordination is maintained in order to ensure that the SBA and the Bank program are mutually reinforcing.

Improving the Investment Climate

10. There is broad consensus that judicial reform is central to improving the business environment in FYR Macedonia. As recognized in the Government's December 2004 Strategy on the Reform of the Judicial Sector, comprehensive judicial reform is especially urgent to firmly establish the efficient effective and fair enforcement of creditor, contract and property rights. The Bank's Doing Business report has also highlighted the time and cost of enforcing contracts and conducting bankruptcy procedures as particular constraints in FYR Macedonia. IMF SBA benchmarks to analyze and budget for the fiscal effects of judicial reform, enact amendments to the Bankruptcy Law, amend the Law on Misdemeanors to allow administrative bodies to impose sanctions without prior court involvement should complement Bank supported reform efforts. Under the PDPL and the proposed Legal and Judicial Reform project, the Bank will focus on: reducing backlog and delays in court proceedings by 25 percent; improve the enforcement of court judgments; improve the regulatory and implementation framework for bankruptcy cases including the development of a profession of bankruptcy trustees; and increase the speed transparency and fairness of administrative decisions. Ultimately, institution building to support the legislative reform agenda will be crucial, and is likely to take several years. 
11. Support for labor market reform will also require close coordination. A very rigid legislative framework governing labor relations has contributed to a stagnant formal labor market, unemployment rates among the highest among transition economies, limited opportunities for new entrants to the labor market, and a large informal sector. The recently adopted Law on Labor Relations - a prior action for the Fund SBA - will eliminate the most burdensome features of the old law and, over time encourage growth, investment, and a gradual shift in employment from the informal sector to the formal sector, with a concomitant strengthening of workers' rights. Further efforts to strengthen labor market flexibility, including the implementation of the new legislative framework, the establishment of alternative dispute resolution mechanisms, and a renegotiation of collective agreements are expected to be supported the three PDPL operations.

12. Judicial and labor market reforms are being complemented by a range of others measures to strengthen the framework for business activity. the enactment and implementation of a new Business Registration Law and associated regulations will critical to reducing the relatively high costs and time of business entry - with the time required for registration currently exceeding the regional average. The Government is also establishing a permanent body to oversee regulatory reform, including the streamlining of business licensing, permits and inspections. These continuing reforms will be supported over the next few years by the Bank's PDPL operations as well as the BERIS project. Further actions to implement corporate governance and accounting and auditing reports on standards and codes (ROSCs) will also be required, including amendments to the Company Law and Securities Law, and the passage of a new Audit Law. Efforts by the Bank to support the capacity of the National Bank (NBRM) to supervise and regulate the banking sector, and to strengthen compliance with anti-money launder and counter-terrorist financing requirements, will also need close coordination with the IMF, and the SBA also includes benchmarks on the strengthening of risk-based supervision by the NBRM.

\section{Public Sector Transparency and Governance}

\section{Reform of health sector financing will be a key element of both the Bank and IMF} programs. Health sector financing makes up 15 percent of Government expenditure and has historically been an area with significant public financial management risks associated with both pharmaceutical procurement and the less than fully transparent operation of the Health Insurance Fund (HIF). As noted in the SBA documentation, implementation of health sector reform will be a litmus test for the authorities' commitment to fight corruption. Prior actions and performance criteria in the SBA requiring submission to Parliament of relevant legislation to tighten the selection of the HIF Board, implement more transparent budget procedures, and introduce binding budget ceilings for the largest public health institutions will be reinforced through existing and proposed Bank programs, which will also support efforts to further prioritize spending, improve the cost-effectiveness of health interventions, and further strengthen mechanisms for pharmaceutical procurement. 
14. Continued collaboration between the Bank and the IMF will be necessary in regard to the consolidation and rationalization of social insurance contributions with the collection of personal income tax. The Bank supports Government efforts, with IMF assistance, to merge collection over time. At the implementation level, however, rationalization and consolidation of social insurance contributions will need to continue to be coordinated with Bank efforts to support Government reform of the social protection system, including the introduction — when conditions allow—of a second pillar pension scheme.

15. Bank support for civil service reform, including strengthening institutional arrangements for strategic prioritization, should complement IMF efforts to improve fiscal and budget management. Extending civil service reform to the majority of Government employees who are not civil servants will further strengthen meritocratic principles and the professionalism of the public sector. Nevertheless, any potential wage decompression among this group - although not scheduled to begin immediately - will require close coordination with the Fund to ensure that fiscal targets, including ceilings on the wage bill, are adhered to. This will also need to occur within the framework of the Ohrid Framework Agreement, and the associated provisions for the representation of minorities. Similarly, IMF benchmarks regarding the completion and implementation of a functional analysis of line Ministries have been set in close coordination with the Bank, as well as other bilateral and multilateral donors active in public sector reform.

16. The Government has made substantial progress in enacting the legislative framework for decentralization, including passage of the controversial Law on Territorial Reorganization, and adopting a formula for the distribution of VAT revenues between the national Government and the various municipalities. Nevertheless, before municipalities assume responsibility for child care and elderly functions under the first phase of decentralization, a plan for resolving municipal arrears still remains to be agreed. Considerable capacity strengthening at the municipal level will also be required if the second phase of decentralization, including the provision of block grants to municipalities to fund schools, is to be implemented successfully and for service standards to be maintained. This will clearly require close coordination between the Government, the IMF, the bank and other relevant donors.

\section{Summary}

17. FYR Macedonia has made considerable progress in macro-economic management and improving the transparency and operations of the public sector. Although continuing governance and macro-economic challenges remain, there is broad consensus that a stronger focus on structural reform will be required to overcome key impediments to growth and employment. This is reflected in both the IMF and Bank programs. The strong similarities 
between aspects of the IMF SBA and the Bank's proposed adjustment and investment lending program have considerable potential to generate synergies and complementarities. This will, however, require continuing strong collaboration between the Macedonian Government, the Bank and the IMF. 
FYR Macedonia: Selected Social and Demographic Indicators

\begin{tabular}{|c|c|c|c|c|c|}
\hline & $\begin{array}{r}\text { FYR of } \\
\text { Macedonia }\end{array}$ & Albania & Bulgaria & Romania & Greece \\
\hline \multicolumn{6}{|l|}{ Area characteristics } \\
\hline Total land area (sq km) & 25,430 & 27,400 & 110,550 & 230,340 & 128,900 \\
\hline of which: arable land (2002, in percent) & 22.3 & 21.1 & 30.3 & 40.9 & 21.1 \\
\hline Population density (2003, people per sq km) & 80.6 & 115.7 & 70.7 & 94.6 & 85.6 \\
\hline \multicolumn{6}{|l|}{ Population } \\
\hline Total population (2003, in thousands) & 2,049 & 3,169 & 7,823 & 21,744 & 11,033 \\
\hline Average annual population growth (2003, in percent) & 0.5 & 0.6 & -0.6 & -0.3 & 0.3 \\
\hline Life expectancy at birth (2003, in years) & 73.6 & 74.3 & 72.1 & 70.1 & 78.0 \\
\hline Male & 71.2 & 72.1 & 68.7 & 66.3 & 75.4 \\
\hline Female & 76.1 & 76.7 & 75.6 & 74.0 & 80.7 \\
\hline Under 5 -years mortality rate (2003, per 1,000 live births) $1 /$ & 11.0 & 21.0 & 17.1 & 20.0 & 5.0 \\
\hline Crude birth rate (2003, per 1,000 persons) & 13.7 & 17.0 & 8.4 & 9.6 & 9.3 \\
\hline Crude death rate (2003, per 1,000 persons) & 8.9 & 6.1 & 14.3 & 12.2 & 9.4 \\
\hline \multicolumn{6}{|l|}{ Income and poverty } \\
\hline GDP per capita (2003, in U.S. dollars) & 2,254 & 1,943 & 2,103 & 2,429 & 15,867 \\
\hline Poverty rate (2000, in percent) $2 /$ & 22.3 & 25.4 & 12.8 & 21.5 & $\ldots$ \\
\hline Urban & 18.8 & 17.2 & $\ldots$ & 20.4 & $\ldots$ \\
\hline Rural & 27.2 & 36.8 & $\ldots$ & 27.9 & $\ldots$ \\
\hline \multicolumn{6}{|l|}{ Health } \\
\hline Physicians (2001, per 1,000 persons) 3/ & 2.2 & 1.4 & 3.4 & 1.9 & 4.4 \\
\hline Hospital beds (2001, per 1,000 persons) $4 /$ & 4.9 & 3.3 & 7.2 & 7.5 & 4.8 \\
\hline \multicolumn{6}{|l|}{ Education } \\
\hline \multicolumn{6}{|l|}{ Net enrollment ratios (2001, in percent) } \\
\hline Primary 5/ & 92.3 & 97.2 & 90.4 & 88.4 & 96.8 \\
\hline Secondary 6/ & 81.2 & 73.9 & 86.7 & 80.0 & 84.9 \\
\hline
\end{tabular}

Sources: World Development Indicators, 2005, World Bank; FYR of Macedonia: Statistics Office; Bulgaria: Poverty Assessment Update, 2001, World Bank; World Development Report 2002, World Bank; and Albania: National Strategy for Socio-Economic Development, Medium-term Program of the Albanian Government "Growth and Poverty Reduction Strategy", November 2001.

1/ Data for Bulgaria is for 2002

2/ Percentage of population below the national poverty line. For FYR Macedonia, the poverty rate or the incidence of poverty is the proportion of individuals with an income (consumption) below 70 percent of median monthly 2000 consumption. Data for Romania refers to 1994. For Bulgaria, data refers to 2001 and the poverty rate is the proportion of individuals with consumption below two-thirds of median consumption in 1997. Data for urban Albania refers to 2002.

3/ Data for Albania is for 2000.

4/ Data for Albania is for 2000, data for Macedonia is for 2002, and data for Greece is for 1999.

5/ Data for Albania is for 2000.

6/ Data for Albania and Greece is for 2000. 
FYR Macedonia: Millennium Development Goals

Goal 1. Eradicate extreme poverty and hunger
Target 1: Halve, between 1990 and 2015 , the proportion of people whose
is less than one dollar a day.
1. Population below US $\$ 1$ a day (percent)
2. Poverty gap ration at US\$1 a day (percent)
3. Share of income or consumption held by poorest 20 percent (percent)

Target 2: Halve, between 1990 and 2015, the proportion of people suffering hunger

4. Prevalence of child malnutrition (percent of children under 5)

5. Population below minimum level of dietary energy consumption (percent)

1990

1995

2001

2002

2003

\section{Goal 2. Achieve universal primary education}

Target 3: Ensure that, by 2015, children will be able to complete a full course of primary schooling.

6. Net primary enrollment ratio (percent of relevant age group)

7. Percentage of cohort reaching grade 5

8. Youth literacy rate (percentage ages 15-24)

\section{Goal 3. Promote gender equality}

Target 4: Eliminate gender disparity in primary and secondary education preferably by 2005 and to all levels of education by 2015 .

9. Ratio of girls to boys in primary and secondary education (percent)

10. Ratio of young literate females to males (percent ages 15-24)

11. Share of women employed in the nonagricultural sector (percent)

12. Proportion of seats held by women in the national parliament (percent)

\section{Goal 4. Reduce child mortality}

Target 5: Reduce by two-thirds, between 1990 and 2015, the under-five mortality rate.

13. Under-five mortality rate (per 1,000)

14. Infant mortality rate (per 1,000 lie births)

15. Immunization against measles (percent of children under 12-months)

$\begin{array}{rrrrr}41.0 & 28.0 & 26.0 & \ldots & 11.0 \\ 34.0 & 24.0 & \ldots & \ldots & 10.0 \\ \ldots & 97.0 & 92.0 & 98.0 & 96.0\end{array}$

Goal 5. Improve maternal health

Target 6: Reduce by three-quarter, between 1990 and 2015, the maternal mortality ration.

16. Maternal mortality ratio (modeled estimate, per 100,000 live births)

17. Proportion of births attended by skilled health personnel

$\begin{array}{rrrrr}98.6 & 97.8 & 98.1 & \ldots & \ldots \\ \ldots & \ldots & \ldots & \ldots & \ldots \\ 38.3 & 38.5 & 41.9 & \ldots & 41.8 \\ \ldots & \ldots & \ldots & \ldots & 18.0\end{array}$

Goal 6. Combat HIV/AIDS, malaria and other diseases

Target 7: Halt by 2015, and begin to reverse, the spread of HIV/AIDS

18. HIV prevalence among females (percent ages 15-24)

19. Contraceptive prevalence rate (percent of women ages 15-49)

20. Number of children orphaned by HIV/AIDS 
FYR Macedonia: Millennium Development Goals

\begin{tabular}{|c|c|c|c|c|c|}
\hline & 1990 & 1995 & 2001 & 2002 & 2003 \\
\hline 21. Incidence of tuberculosis (per 100,000 people) & $\ldots$ & $\ldots$ & 35.0 & 41.5 & 31.4 \\
\hline \multicolumn{6}{|l|}{$\underline{\text { Goal 7. Ensure environmental sustainability }}$} \\
\hline \multirow{3}{*}{\multicolumn{6}{|c|}{$\begin{array}{l}\text { Target 8: Integrate the principles of sustainable development into policies and program. } \\
\text { Reverse the loss of environmental resources. Halve, by } 2015 \text {, the proportion of people without } \\
\text { sustainable access to safe drinking water. By } 2020 \text {, to have achieved a significant improvement } \\
\text { in the lives of at least } 100 \text { million slum dwellers. }\end{array}$}} \\
\hline & & & & & \\
\hline & & & & & \\
\hline 23. Forest area (percent of total land area) & 35.6 & $\ldots$ & 35.6 & $\ldots$ & $\ldots$ \\
\hline 24. Nationally protected areas (percent of total land area) & $\ldots$ & 7.1 & 7.1 & 7.1 & 7.1 \\
\hline 26. $\mathrm{CO} 2$ emissions (metric tons per capita) & 5.5 & 5.4 & 5.5 & $\ldots$ & $\ldots$ \\
\hline 27. Access to an improved water source (percent of population) & $\ldots$ & $\ldots$ & $\ldots$ & $\ldots$ & $\ldots$ \\
\hline 28. Access to improved sanitation (percent of population) & $\ldots$ & $\ldots$ & $\ldots$ & $\ldots$ & $\ldots$ \\
\hline 29. Access to secure tenure (percent of population) & $\ldots$ & $\ldots$ & $\ldots$ & $\ldots$ & $\ldots$ \\
\hline
\end{tabular}

Goal 8. Develop a global partnership for development

Target 9: Develop and implement strategies for productive work for youth

30. Youth unemployment rate (percent of total labor force ages 15-24)

31. Fixed line and mobile telephones (per 1,000 people)

$\begin{array}{rrrrr}\cdots & \cdots & \cdots & \cdots & \cdots\end{array}$

32. Personal computers (per 1,000 people)

\section{$\underline{\text { General Indicators }}$}

Population

Gross national income (\$)

GNI per capita

Adult literacy rate (percent of people ages 15 and above)

Total fertility rate (births per woman)

Life expectancy at birth (years)

Aid (percent of GNI)
1.9 million 2.0 million 2.0 million 2.0 million 2.0 million

3.4 billion 3.4 billion 3.4 billion 3.5 billion 3.5 billion

$\begin{array}{lllll}1,750.0 & 1,710.0 & 1,690.0 & 1710.0 & 1980.0\end{array}$

$\begin{array}{llllll}\ldots & \ldots & \ldots & \ldots & \ldots \\ 2.1 & \ldots & \ldots & \ldots & \end{array}$

$\begin{array}{lllll}2.1 & 2.0 & 1.8 & 1.8 & 1.8\end{array}$

$\begin{array}{lllll}71.6 & 71.9 & 72.8 & 73.4 & 73.6\end{array}$

Sources: World Development Indicators database, April 2004. 


\section{Statement by the IMF Representative on Former Yugoslav Republic of Macedonia \\ August 31, 2005}

This statement provides information on developments since the issuance of the staff report for Former Yugoslav Republic of Macedonia' Request for Stand-By Arrangement and Extension of Repurchase Expectations. It does not change the thrust of the staff appraisal.

On August 23, 2005 Standard \& Poor's raised FYR Macedonia's sovereign credit ratings. In upgrading the ratings the agency cited progress towards political stabilization, labor market and judicial reforms, sustained macroeconomic stability, and downward trending debt levels. The long-term foreign currency rating was raised from $\mathrm{BB}$ to $\mathrm{BB}+$ while the long-term local currency rating was raised from $\mathrm{BB}+$ to $\mathrm{BBB}-$, which is investment grade. The short-term local currency rating was raised from $\mathrm{B}$ to $\mathrm{A}-3$ while the short-term foreign currency rating was kept at B. 
Press Release No. 05/196

FOR IMMEDIATE RELEASE

September 1, 2005
International Monetary Fund

Washington, D.C. 20431 USA

\section{IMF Executive Board Approves Three-Year US\$75.8 Million Stand-By Arrangement for Macedonia}

The Executive Board of the International Monetary Fund (IMF) today approved a three-year SDR 51.7 million (to about US\$75.8 million) Stand-By Arrangement for the former Yugoslav Republic of Macedonia to support the country's economic program and approved an extension to the obligations schedule of the country's repayments to the Fund in the total amount of SDR 5.4 million (about US\$7.9 million).

The approval of the arrangement enables FYR Macedonia to draw SDR 10.5 million (about US\$15.4 million) immediately. The authorities have indicated that, after making the initial purchase, they do not plan to draw under the arrangement and intend to treat the arrangement as precautionary.

Following the Executive Board's discussion on Macedonia, Mr. Takatoshi Kato, Deputy Managing Director and Acting Chair, stated:

"The authorities of the former Yugoslav Republic of Macedonia are to be commended for their sound macroeconomic policies: fiscal discipline has kept the debt ratio low, while the de facto exchange rate peg has brought inflation close to zero. However, structural impediments have led to relatively weak economic growth, persistently high unemployment, and a wide current account deficit. The authorities' strong program focuses on structural reforms aimed at increasing growth and employment and reducing the external imbalance, while securing the past gains in macroeconomic stabilization. The program also supports the process of integration with the EU, and is expected to allow for an orderly exit from longterm financial engagement with the Fund. The recent upgrading in FYR Macedonia's credit ratings is encouraging.

"A core program element is an ambitious labor market reform, which should bring down non-wage labor costs and allow for a more efficient allocation of resources. The reform should help draw employment out of the grey market, thus ensuring that labor enjoys legal protections while also contributing to increased revenue collection. A comprehensive judicial reform to strengthen the rule of law and bring the court system closer to European standards will further improve the business climate. These reforms and other measures in the program 
should lead to higher investment, including foreign direct investment, and lay the basis for stronger growth and employment creation.

"The program's fiscal policies will sustain the adjustment already achieved, keeping the government debt ratio on a declining path, including through the planned broad-based containment of expenditures. Structural fiscal reforms, including the consolidation and rationalization of the collection of payroll taxes, and measures to improve budget planning and execution, will strengthen the efficiency of government operations over time. In the same vein, the authorities have begun to confront long-standing problems in the health sector, signaling a renewed commitment to fiscal integrity and budget management in this sector.

"Monetary policy will continue to rely on the de facto peg of the denar to the euro to ensure stable and low inflation. The robustness of the peg will be underpinned by using privatization receipts to boost international reserves and by recapitalizing the central bank in order to ensure its financial independence and further strengthen its policy credibility. Supervisory procedures and regulations have already been strengthened in order to contain balance sheet risks that might arise from euroization and rapid credit growth. Further prudential measures to manage banks' balance sheet risks are included in the program," Mr. Kato said. 


\section{Background}

Despite the success in macroeconomic stabilization under the previous IMF-supported arrangement, which expired in August 2004, economic growth in FYR Macedonia has remained modest. Fiscal discipline has produced a modest and declining debt ratio and the de facto exchange rate peg has brought inflation close to zero, but deficiencies in the functioning of the relevant institutions have undermined business activity and kept per capita FDI low. The resulting weak competitiveness has led to disappointing growth, high unemployment, and a persistent current account deficit.

\section{Program Summary}

The new program includes structural reforms to improve the business climate, and thus competitiveness, and sound macroeconomic policies to maintain financial stability. As the structural reforms take effect, GDP growth is projected to accelerate from less than 3 percent per annum in 2003-04 to 4.5 percent in 2007 and the external current account deficit is expected to narrow significantly.

On the macroeconomic front, monetary policy will continue to be based on the de facto peg to the euro, ensuring continued low inflation. The peg will be strengthened by using privatization proceeds to raise international reserve cover from about three months of imports at end-2004 to about four months of imports by end 2006. Appropriate financial policies will maintain reserve cover thereafter. Given recent rapid credit growth - much of it denominated in, or indexed to, the euro - the program aims to mitigate balance sheet risks through strengthened prudential oversight and measures aimed at improving governance in banks.

The fiscal program is anchored by a medium-term deficit target of 0.6 percent of GDP, which will bring the central government debt-to-GDP ratio down to about 33 percent by 2008 . The key to reaching the program's ambitious deficit target is a broad-based containment of expenditures, including wages, and the strengthening of tax administration through a carefully sequenced set of reforms. Expenditure management will be improved and reform of the Health Insurance Fund will strengthen government oversight over health expenditure. The program also aims to improve FYR Macedonia's borrowing capacity by developing the domestic debt market and establishing access to international capital markets.

The structural core of the program is a wide range of reforms which should enhance competitiveness by improving conditions for investment and business activity. The new Law on Labor Relations, which strikes a better balance between workers' rights and employers' needs, creates more favorable conditions for business activity by reducing restrictions on short-term and part-time employment contracts and overtime work. Comprehensive judicial reform, to be implemented over several years, will create a fairer and more predictable framework for business activity by increasing the independence and professionalism of judges, eliminating court delays, and removing misdemeanors and administrative cases from the regular courts. The labor market and judicial reforms will be complemented by a number 
of measures to strengthen the framework for business activity, including, notably, measures to shorten the time needed to open a business. Many of the reforms included in the program are also supported by the World Bank and by other multilateral and bilateral donors. 
FYR Macedonia: Selected Economic Indicators, 2003-08

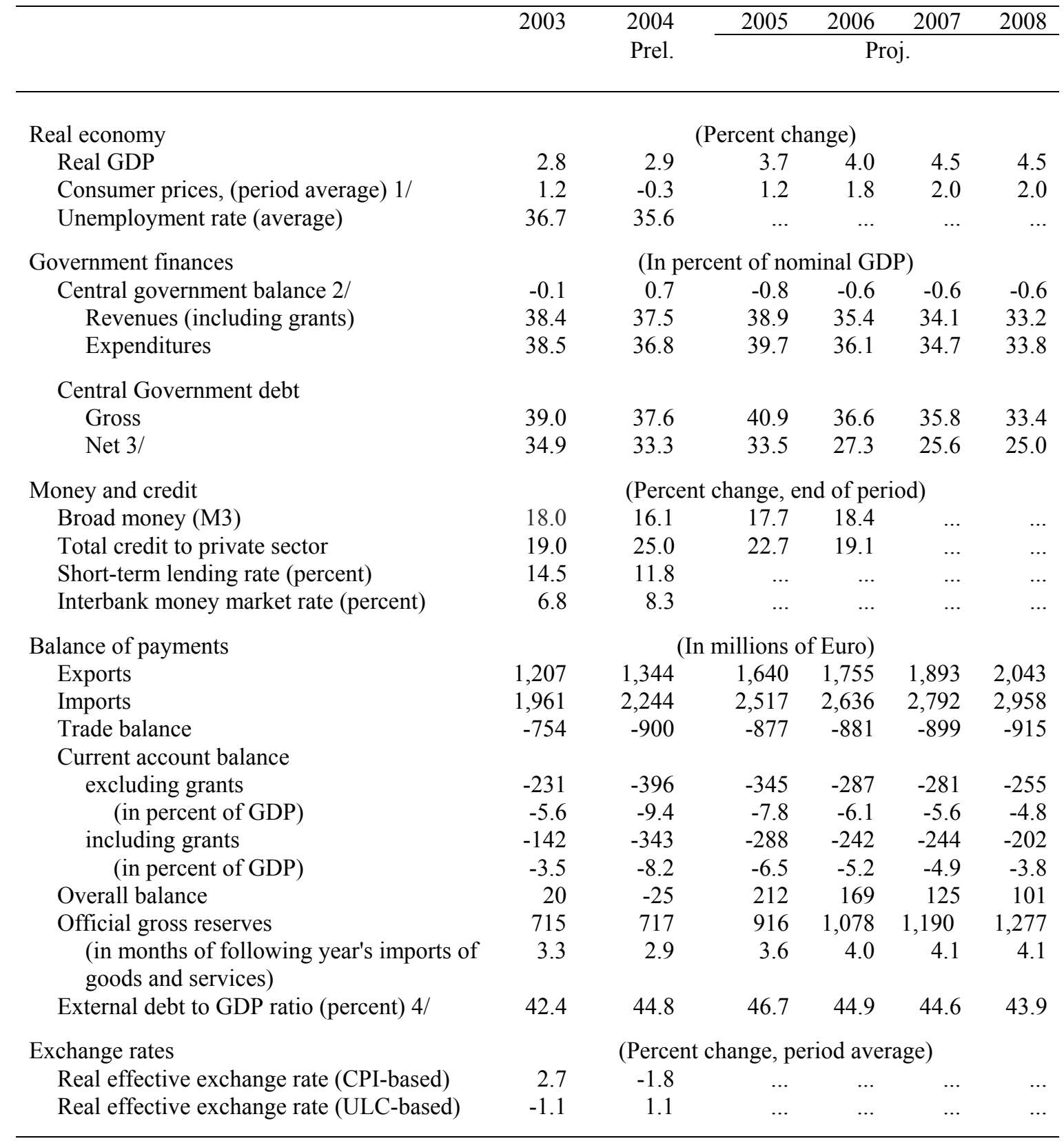

Sources: Data provided by the authorities; and IMF staff projections.

1/ Recent revisions by the State Statistics Office have not been incorporated in this document.

2/ 0.4 percent of GDP of the 2005 fiscal account deficit is caused by the NBRM recapitalization.

$3 /$ Gross debt minus government's deposits with the NBRM.

4/ Total external debt, including trade credit. Includes for 2005 an $€ 150$ million Eurobond issuance. 


\section{Statement by Jeroen Kremers, Executive Director for the Former Yugoslav Republic of Macedonia August 31, 2005}

The Macedonian authorities would like to express their appreciation for staff's comprehensive report and the candid exchange of views during the discussions of the economic program for 2005-2008 proposed to be supported by a new Stand-By Arrangement. Macedonia's record of macroeconomic stabilization has been advanced and the main objective of the previous SBA - a return to a sustainable fiscal position after the 2001 security crises - has been attained. By implementing all prior actions in a timely and consistent manner, the authorities have reconfirmed their strong commitment to sound economic and financial policies. Against this background, they request support for a threeyear SBA and Extension of Repurchase Expectations.

The authorities attach great value to a close relationship with the Fund and the requested program is designed to sustain macroeconomic stability, to enhance domestic and international credibility, increase investors confidence, and to improve external competitiveness and accelerate growth. In this vein, the authorities' ambitious program includes structural reforms aimed at addressing the root causes of Macedonia's modest growth performance. The authorities believe that the proposed program realistically reflects the recent economic developments in Macedonia and the challenges ahead, and, as always, consent to the publication of the report.

The new economic program represents a new stage in Macedonia's transition to a market economy, its relations with the International Financial Institutions, including the IMF, and its further integration in the global economy. Recent economic and political developments have marked a gradual but consistent shift to sustained macroeconomic stability, as reflected by the March 2005 local elections, which confirmed the progress in implementing the Framework Agreement. Furthermore, recent international engagement, such as the application for NATO membership and European Union candidacy, as well as key reforms supported by the proposed program enjoy broad support across society. This gives confidence that the next parliamentary elections will not disrupt the reform agenda.

The August 23 Standard \& Poor's upgrade of Macedonia's long-term foreign and local currency sovereign credit ratings ${ }^{1}$ reflects confidence in the ongoing progress of structural reforms, macroeconomic stability, and the downward trend of Macedonia's public debt levels.

\footnotetext{
${ }^{1}$ To 'BB+' from 'BB', and to 'BBB-' (investment grade) from 'BB+', respectively, the short-term local currency rating to 'A-3' from 'B', and affirmation of the 'B' short-term foreign currency rating.
} 
Notably, fiscal consolidation contributed to the improved economic situation from 2003 onwards, supported by price stability and a stable exchange rate. Inflation has been retained at low single digits since the mid-1990s, reaching a very low level in recent years. The economic growth achieved over the last two years, though still below potential, is expected to increase to $3 \frac{3}{4}$ percent in 2005 , and further accelerate to $4 \frac{1}{2}$ percent under the new program between 2005-08. Staff estimates that the acceleration in GDP growth will be accompanied by a reduction of the external current account imbalance from 9.4 percent of GDP in 2004 to 4.8 percent of GDP in 2008.

\section{Fiscal policy}

Following a tight fiscal stance in 2003, the authorities realized an impressive consolidation by undertaking politically difficult measures, among others, increasing taxes, raising utility prices and reducing expenditures. The central budget deficit was significantly reduced from 5.3 percent of GDP in 2002 to 0.9 percent of GDP in 2003 and a surplus in 2004. For 2005, the authorities have passed a supplementary budget with a deficit of 0.4 percent, which is in line with the medium-term fiscal program. This represents a substantial tightening relative to the original budget deficit target of 1.2 percent of GDP. Although pressures to increase expenditures persist, the authorities remain committed to maintain fiscal discipline, in light of the large current account deficit, financing constraints and uncertain future expenditures.

The proposed program builds on the successful fiscal adjustment under the previous SBA by targeting a medium-term central government deficit ( 0.6 percent of GDP) that will reduce the public debt ratio. The debt sustainability analysis shows that debt dynamics are generally favorable. Against this background, the authorities' financial program aims at managing the transition from dependence on official balance of payments support to reliance on market financing, while maintaining the fixed exchange rate as anchor for price stability. In this regard, the authorities are fully aware that the successful transition to market financing will require not only fiscal prudence, but also the development of a debt management strategy, and have established a Public Debt Management Department in the Ministry of Finance. The authorities' initial strategy focuses on the development of the domestic security market and entering the Eurobond market later this year.

An important element of the broader fiscal reform agenda is the implementation of the comprehensive reform of the tax administration in line with FAD recommendations. In this area, the authorities intend to broaden the tax base by cutting back on tax incentive schemes, harmonizing the base and unifying the collection of personal income tax and social contribution. The implementation of these critical measures will be closely monitored by the Fund, including through benchmarks and performance criteria, consistently spread over the life of the program. To this end, the government has approved the new Law on Tax Administration Procedures, which is envisaged to be passed by the Parliament by the end of the year. This will improve the efficiency of the Public Revenue Office (PRO) and broaden its authority, allowing it to impose penalties and seize assets without requiring prior court action. 
The authorities plan to improve the use of scarce administrative resources in the collection of the social insurance contributions. They will gradually consolidate the collection activities of the pension, health and unemployment funds and the PRO. By mid2006, a new Large Contributor Office will be created, comprising separate units for the Pension and Disability Fund (PDF) and the Health Insurance Fund (HIF) (structural benchmark).

On the expenditure side, the authorities plan to keep public wages and public hiring under strict control to ensure that the share of salaries in tax revenues is maintained at the level of the 2005 supplementary budget. In line with previous Board recommendations, steps have been taken to increase the efficiency, transparency and fiscal soundness of the health care system. In this connection, as from May 2005, monthly HIF budget execution and financial reports of the seven largest Public Health Institutions (PHI) have been published on the HIF website, and starting with the 2006 budget, the HIF will report on budget execution, including transfers to PHI's. To further improve transparency and financial accountability of the system, the seven large PHI's will prepare budgets for 2006, for approval by the Ministry of Health in consultation with the HIF and the Ministry of Finance (performance criterion).

\section{Monetary policy and exchange rate regime}

The National Bank of the Republic of Macedonia (NBRM) conducts a monetary policy that, in combination with the tight supplementary budget and expected inflows, will allow a modest increase in the official foreign exchange reserves. The increase in reserves and proceeds from the Eurobond issue are expected to result in an increase in the import coverage from 2.9 months in 2004 to 3.6 months by the end of 2005 . Broad money will grow in line with the recent trends in velocity, and the level of euroization is expected to remain constant. The authorities believe that this will allow meeting the reserves target without need for increasing interest rates. However, the NBRM stands ready to use all necessary instruments to meet the program targets.

The staff reports that the authorities considered the option of a more flexible exchange rate regime and recognize the merit of this exercise. However, they would like to reiterate that the peg has served the country well as a clearly defined monetary anchor, and will continue to do so. In examining the alternatives, the NBRM has the view that inflation or monetary aggregate targeting would be premature given the emerging financial market and the unpredictability of the monetary transmission mechanism.

Under the new program, the independence of the central bank will be further strengthened by increasing its capital base and establishing rules that will help maintain its financial stability. In this regard, the authorities have agreed to a package aimed at underpinning its capital base and improving its profitability.

\section{Financial sector development}

The development of the treasury securities market is a core priority of the program because it affects the government's capacity to borrow, the scope for central bank open 
market operations and the investment opportunities for firms, households and banks. In order to develop this market, the NBRM is promoting secondary trading and the introduction of repurchase agreements. Also, by the end of the year, the government and the NBRM will agree on a plan to phase out central bank bills and use treasury securities for open market operation (structural benchmark). The central bank has finalized all legal and accounting requirements for introducing repurchase agreements with treasury bills, and it welcomes IMF technical assistance on issues concerning repo transactions.

The staff correctly notes that, while continued credit growth is a positive development, there is a need for careful management of the implied risks. To this end, the authorities concur with staff that greater attention should be paid to strengthening banking supervision. The NBRM has already assessed borrowers' foreign exchange risk exposure as part of its regular supervision activities. Looking forward, the Macedonian authorities will undertake measures to strengthen banking sector governance. As a first step, they will amend the Law on Banks to give the NBRM authority to enforce proper requirements on existing bank shareholders and to strengthen procedures for bankruptcy. Since strengthening of the financial sector is a core element of the program, the amendment of the Banking Law is a prior action for the second review, and the development of the Supervisory Development Plan a structural benchmark.

\section{Structural reforms}

The proposed program relies on ambitious structural reforms aimed at raising productivity growth, fostering entrepreneurial activity and eliminating rigidities in the labor market. The overarching objective of the authorities' medium-term program, which is in line with the 2004 Ex Post Assessment, is to increase growth and employment, while at the same time reducing the internal and external imbalances, and safeguarding past gains in macroeconomic stabilization.

One of the centerpieces of the reform program, next to the health reform as discussed above, is the recently-adopted Law on Labor Relations, which, as staff correctly points out, marks a turning point in Macedonia's labor relations. Its main purpose is to make the labor market more flexible. This law is expected, over time, to have a large impact on growth, foreign investment and the reduction of the shadow economy.

Another critical structural project, which the authorities believe will have a major impact on the business environment, is the ambitious and comprehensive judicial reform program. This program, which will be implemented over the course of several years, aims at strengthening the rule of law by conforming to European standards. Its first stage consists of legislative actions to create a legal and constitutional basis for the new court system. The second stage consists of administrative reforms: improved court administration and budgeting, and the creation of a new enforcement agency. The elaboration of such a comprehensive strategy was possible only with the extensive technical assistance received from donors, including EU institutions and member countries, NATO, and USAID, which is highly appreciated by the Macedonian authorities. 
Looking forward, the authorities are confident that the positive outcomes of the labor market and judicial reforms will be complemented by a number of measures to improve the business climate. Such measures will include, among others, reduction in the time and cost needed to open a business; reduction of the excessive number of licensing requirements, improvement of legislative procedures and the institutional framework for corporate governance, and strengthening the institutional framework for bankruptcy and measures to improve land registration.

The authorities' reform agenda also includes an ambitious privatization program extended to the electricity sector, telecommunications, financial sector and retail property. A partial privatization of the Electricity power company of Macedonia (ESM) are in the final preparation process with support from the EBRD, and the World Bank, which is providing the assessment of the regulatory implications. Furthermore, the government intends to sell its residual shares in the telecommunication sector by mid-2006, and will start tendering its equity in commercial banks by the end of the year.

Finally, I would like to note that the authorities intend to treat the proposed SBA as precautionary after making the initial purchase. The request for an extension of repurchase expectations totaling SDR 5.4 million falling due before end-September 2006 is based on the external financing needs during the next twelve months and the deterioration of the external position relative to the outlook in March, 2003, when the last SBA was approved. 